JOURNAL OF

SYMPLECTIC GEOMETRY

Volume 1, Number 3, 543-645, 2002

\title{
The symplectic vortex equations and invariants of Hamiltonian group actions
}

\author{
Kai Cieliebak $^{1}$, A. Rita Gaio ${ }^{2}$, \\ Ignasi Mundet i Riera, Dietmar A. Salamon
}

In this paper we define invariants of Hamiltonian group actions for central regular values of the moment map. The key hypotheses are that the moment map is proper and that the ambient manifold is symplectically aspherical. The invariants are based on the symplectic vortex equations. Applications include an existence theorem for relative periodic orbits, a computation for circle actions on a complex vector space, and a theorem about the relation between the invariants introduced here and the Seiberg-Witten invariants of a product of a Riemann surface with a two-sphere.

\section{Introduction.}

In this paper we study the vortex equations with values in a symplectic manifold $(M, \omega)$. We assume that $(M, \omega)$ is equipped with a Hamiltonian action by a compact Lie group $\mathrm{G}$ that is generated by an equivariant moment map

$$
\mu: M \rightarrow \mathfrak{g} .
$$

The symplectic vortex equations have the form

$$
\bar{\partial}_{J, A}(u)=0, \quad * F_{A}+\mu(u)=\tau .
$$

Here $P \rightarrow \Sigma$ is a principal G-bundle over a compact Riemann surface, $u: P \rightarrow M$ is an equivariant smooth function, and $A$ is a connection on $P$. To define the terms in (1) we must fix a G-invariant almost complex structure on $M$, a Riemannian metric on $\Sigma$, and an element $\tau \in Z(\mathfrak{g})$ in the center of the Lie algebra. The expression $\bar{\partial}_{J, A}$ denotes the nonlinear Cauchy-Riemann operator, twisted by the connection $A$, and $*$ denotes the

\footnotetext{
${ }^{1}$ Partially supported by National Science Foundation grant DMS-0072267.

${ }^{2}$ Partially supported by the FCT grant PRAXIS XXI/BPD/22084/99.
} 
Hodge $*$-operator on $\Sigma$. Equations (1) were introduced in $[6,14,25]$. In the physics literature these equations are known as gauged sigma models in the case where the target space $M$ is a complex vector space. Special cases of the symplectic vortex equations include pseudoholomorphic curves in symplectic manifolds $(\mathrm{G}=\{\mathbb{1}\})$, the usual vortex equations over $\Sigma(M=\mathbb{C}$ with the standard $S^{1}$-action $\left.[3,16]\right)$, Bradlow pairs $\left(M=\mathbb{C}^{2}\right.$ with the standard $\mathrm{U}(2)$-action [4, 36]), anti-self-dual instantons over a product $\Sigma \times S(M$ is the infinite dimensional space of $\mathrm{SO}(3)$-connections over $S$ and $\mathrm{G}$ is the gauge group), and the Seiberg-Witten equations over $\Sigma \times S$ ( $M$ is the space of pairs, each consisting of a connection on a line bundle $L \rightarrow S$ and a holomorphic section, and $\mathrm{G}$ is the gauge group of $L$ ). In the present paper the symplectic manifold $M$ is always finite dimensional.

In the Kähler case the symplectic vortex equations admit an algebro geometric interpretation. For example, if $M$ is a complex vector space then the map $u$ defines a holomorphic section of a vector bundle over $\Sigma$ and the solutions of (1) correspond to stable pairs.

We impose the following conditions on the triple $(M, \omega, \mu)$.

(H1) The moment map $\mu$ is proper.

(H2) There exists a (strongly) convex structure $(f, J)$ on $M$. This means that $J$ is a G-invariant and $\omega$-compatible almost complex structure on $M, f: M \rightarrow[0, \infty)$ is a proper G-invariant function, and there exists a continuous function $Z(\mathfrak{g}) \rightarrow \mathbb{R}: \tau \mapsto c(\tau)$ such that

$$
f(x) \geq c(\tau) \Longrightarrow \quad \begin{aligned}
& \left\langle\nabla_{v} \nabla f(x), v\right\rangle+\left\langle\nabla_{J v} \nabla f(x), J v\right\rangle \geq 0 \\
& \quad d f(x) J(x) L_{x}(\mu(x)-\tau) \geq 0
\end{aligned}
$$

for every $x \in M, v \in T_{x} M$, and $\tau \in Z(\mathfrak{g})$. Here $\nabla$ denotes the LeviCivita connection of the metric $\langle\cdot, \cdot\rangle=\omega(\cdot, J \cdot)$ and $L_{x}: \mathfrak{g} \rightarrow T_{x} M$ denotes the infinitesimal action.

(H3) The manifold $(M, \omega)$ is symplectically aspherical, i.e.

$$
\int_{S^{2}} v^{*} \omega=0
$$

for every smooth function $v: S^{2} \rightarrow M$.

Hypothesis (H2) is a natural generalization of the existence of a plurisubharmonic function on noncompact symplectic manifolds [12]. Both hypotheses $(H 1)$ and $(H 2)$ are natural in the context of this paper and are needed 
to obtain any kind of compactness theorem for the solutions of (1). Hypothesis (H3) constitutes a more severe restriction and should in the future be removed or weakened. It implies that $M$ is noncompact whenever there exists a G-orbit of positive dimension. However, there are many interesting examples where all three hypotheses are satisfied, e.g., linear actions on $\mathbb{C}^{n}$ with proper moment maps [6]. The three hypotheses together guarantee that the moduli space of gauge equivalence classes of solutions of (1) is compact. As a result one can use these moduli spaces to define invariants which are analogous to the Gromov-Witten invariants in the nonequivariant case. Let $B \in H_{2}^{\mathrm{G}}(M ; \mathbb{Z})$ denote the equivariant homology class represented by the map $u$. Then the invariants take the form of a homomorphism

$$
\Phi_{B, \Sigma}^{M, \mu-\tau}: H_{\mathrm{G}}^{*}(M ; \mathbb{Q}) \rightarrow \mathbb{Q}
$$

whenever $\tau$ is a central regular value of $\mu$. This homomorphism takes integer values on integral cohomology classes whenever $\mathrm{G}$ acts freely on $\mu^{-1}(\tau)$. It depends only on the component of $\tau$ in the open set of central regular values. We emphasize that the complex structure on $\Sigma$ is fixed in the definition of our invariant. There should be natural extensions which involve varying complex structures on the domain $\Sigma$ and dispense with hypothesis $(H 3)$. However, the definition of the invariants in these cases will probably require a considerable amount of nontrivial analysis. For first steps in this direction see $[25,26]$.

As a first application we establish the existence of relative periodic orbits for time dependent G-invariant Hamiltonian systems. This can be viewed as an equivariant version of a theorem of Gromov [17].

Theorem A. Assume $(H 1-3)$ and let $\tau \in Z(\mathfrak{g})$ be a central regular value of $\mu$ such that $\mu^{-1}(\tau) \neq \emptyset$. Then every time-dependent 1 -periodic $\mathrm{G}$-invariant Hamiltonian system admits a contractible relative periodic orbit in $\mu^{-1}(\tau)$.

If $\mathrm{G}$ is abelian then the hypothesis that $\tau$ is a regular value can be dropped and we obtain a contractible relative periodic orbit on every nonempty level set of $\mu$. It is natural to conjecture that this should continue to hold under hypothesis $(H 1)$ only. Our proof of Theorem A follows closely Gromov's argument in [17] for the nonequivariant case. The pseudoholomorphic curves in Gromov's proof are replaced by the solutions of the perturbed symplectic vortex equations.

In some cases the invariants can be computed explicitly. We carry out such a computation for linear circle actions on $\mathbb{C}^{n}$. Suppose $S^{1}$ acts on $\mathbb{C}^{n}$ with positive weights $\ell_{1}, \ldots, \ell_{n}$ and denote the correponding moment map 
by $\mu_{\ell}$. Then there is only one nontrivial chamber for the regular values of $\mu_{\ell}$ and we denote by $\Phi^{\mathbb{C}^{n}, \mu_{\ell}}$ the invariant in this chamber.

Theorem B. Let $\Sigma$ be a compact Riemann surface of genus $g, d \in \mathbb{Z} \cong$ $H_{2}^{S^{1}}\left(\mathbb{C}^{n} ; \mathbb{Z}\right)$ an integer, and $c \in H_{S^{1}}^{2}\left(\mathbb{C}^{n} ; \mathbb{Z}\right) \cong \mathbb{Z}$ the positive generator. Suppose that

$$
m:=\sum_{\nu=1}^{n}\left(d \ell_{\nu}+1-g\right)+g-1 \geq 0 .
$$

Then

$$
\Phi_{d, g}^{\mathbb{C}^{n}, \mu_{\ell}}\left(c^{m}\right)=\left(\sum_{\nu=1}^{n} \ell_{\nu}\right)^{g} \prod_{\nu=1}^{n} \ell_{\nu}^{-\left(d \ell_{\nu}+1-g\right)}
$$

In the case $\ell_{\nu}=1$ and $d>2 g-2$ this was proved by BertramDaskalopoulos-Wentworth [2]. The proof of Theorem B involves the AtiyahSinger index theorem for families of Cauchy-Riemann operators.

Our invariants are related to the Seiberg-Witten invariants of certain four-manifolds. The key observation is that the symmetric product of a Riemann surface $S$ can be interpreted as a symplectic quotient of the infinite dimensional space whose elements are pairs, each consisting of a connection and a holomorphic section of a line bundle $L \rightarrow S$ of degree $d$. In this situation the symplectic vortex equations, with $M$ replaced by the infinite dimensional space of which $\operatorname{Sym}^{d}(S)$ is a quotient, are the Seiberg-Witten equations on $\Sigma \times S$. When $d>2 g_{S}-2$ one can write the symmetric product as a quotient of a finite dimensional symplectic manifold $M_{d, S}$ (called the vortex manifold of the pair $(d, S)$ ) by a Hamiltonian $S^{1}$ action with a moment map $\mu_{d, S}$ which satisfies $(H 1-3)$. The following theorem relates the invariants of $\left(M_{d, S}, \mu_{d, S}\right)$ to the Seiberg-Witten invariants. It is a special case of a result for general ruled surfaces in [27].

Theorem C ([27]). Let $S$ be the Riemann sphere and $\Sigma$ be a compact Riemann surface of genus $g$. Let $d$ and $k$ be nonnegative integers such that

$$
m:=d(1-g)+(d+1) k \geq 0 .
$$

Then

$$
\Phi_{k, \Sigma}^{M_{d, S}, \mu_{d, S}}\left(c^{m}\right)=\operatorname{SW}_{\Sigma \times S}\left(\gamma_{k, d}\right),
$$

where $\gamma_{k, d}$ denotes the spin ${ }^{c}$-structure determined by $k$ and $d$. Moreover, if

$$
k>2 g-2,
$$


then

$$
\Phi_{k, \Sigma}^{M_{d, S}, \mu_{d, S}}\left(c^{m}\right)=\Phi_{d, S}^{M_{k, \Sigma}, \mu_{k, \Sigma}}\left(c^{m}\right) .
$$

Combining Theorems $\mathrm{B}$ and $\mathrm{C}$ one can recover the computation of the Seiberg-Witten invariants of product ruled surfaces by Li-Liu [21] and OhtaOno [28].

It is also interesting to examine the relation between our invariants and the Gromov-Witten invariants of the symplectic quotient

$$
\bar{M}:=M / / \mathrm{G}(\tau):=\mu^{-1}(\tau) / \mathrm{G}
$$

whenever $\mathrm{G}$ acts freely on $\mu^{-1}(\tau)$. Such a relation was established in [15] under the hypothesis that the quotient is monotone. Under this condition (and hypotheses $(H 1-3)$ ) it is shown in [15] that there exists a surjective ring homomorphism

$$
\phi: H_{\mathrm{G}}^{*}(M) \rightarrow \mathrm{QH}^{*}(\bar{M})
$$

(with values in the quantum cohomology of the quotient) such that

$$
\Phi_{B, \Sigma}^{M, \mu-\tau}(\alpha)=\mathrm{GW}_{\bar{B}, \Sigma}^{\bar{M}}(\phi(\alpha))
$$

for every $\alpha \in H_{\mathrm{G}}^{*}(M)$ and every $\bar{B} \in H_{2}(\bar{M} ; \mathbb{Z})$, where $B$ denotes the image of $\bar{B}$ under the homomorphism $H_{2}(\bar{M} ; \mathbb{Z}) \rightarrow H_{2}^{\mathrm{G}}(M ; \mathbb{Z})$. The proof is based on an adiabatic limit analysis which relates the solutions of the symplectic vortex equations in $M$ to pseudoholomorphic curves in the symplectic quotient $\bar{M}$. This analysis is analogous to the proof of the Atiyah-Floer conjecture in [11].

The present paper is organized as follows. In Section 2 we discuss the basic properties of solutions to the symplectic vortex equations such as the energy identity, unique continuation, and apriori estimates under the convexity hypothesis (H2). Section 3 establishes the basic compactness and regularity theorems and Section 4 discusses the Fredholm theory. In Section 5 we establish the integer invariants under the hypothesis that $\mathrm{G}$ acts freely on $\mu^{-1}(\tau)$. Section 6 is of preparatory nature. In it we recall some background from [7] about the equivariant Euler class of G-moduli problems. Section 7 establishes the rational invariants in the presence of finite isotropy and discusses some relations between the invariants. Theorems A, B, and C are proved in Sections 8, 9, and 10. Appendix A establishes existence and uniqueness for a coupled Kazdan-Warner equation that appears in the proof of Theorem C. Appendix B gives a proof of the local slice theorem for gauge group actions in a form needed for the compactness and regularity results of Section 3. 


\section{The geometry of symplectic vortices.}

\subsection{The vortex equations in a symplectic manifold.}

Let $(M, \omega)$ be a (not necessarily compact) symplectic manifold and $\mathrm{G}$ be a compact Lie group which acts on $M$ by symplectomorphisms. Let $\mathfrak{g}=$ Lie $(\mathrm{G})$ denote the Lie algebra and

$$
\mathfrak{g} \rightarrow \operatorname{Vect}(M, \omega): \xi \mapsto X_{\xi}
$$

denote the infinitesimal action. We assume that the action is Hamiltonian. This means that the action is generated by an equivariant map $\mu: M \rightarrow \mathfrak{g}$ that satisfies

$$
\iota\left(X_{\xi}\right) \omega=d\langle\mu, \xi\rangle
$$

for every $\xi \in \mathfrak{g}$. Here $\langle\cdot, \cdot\rangle$ denotes an invariant inner product on $\mathfrak{g}$. The function $\mu$ is called a moment map for the action.

Let $P \rightarrow \Sigma$ be a principal G-bundle over a compact connected oriented Riemann surface $\left(\Sigma, J_{\Sigma}, \operatorname{dvol}_{\Sigma}\right)$. We emphasise that the volume form and the complex structure on $\Sigma$ are fixed. Denote by $C_{\mathrm{G}}^{\infty}(P, M)$ the space of equivariant functions $u: P \rightarrow M$ and by $\mathcal{A}(P)$ the space of connections on $P$. We think of $A \in \mathcal{A}(P)$ as an equivariant Lie algebra valued 1-form on $P$ which identifies the vertical tangent space with $\mathfrak{g}$. Its curvature is a 2 -form $F_{A}$ on $\Sigma$ with values in the associated Lie algebra bundle $\mathfrak{g}_{P}:=P \times_{\text {ad }} \mathfrak{g}$. In this paper we study the following system of nonlinear first order partial differential equations, for pairs $(u, A) \in C_{\mathrm{G}}^{\infty}(P, M) \times \mathcal{A}(P)$,

$$
\bar{\partial}_{J, A}(u)=0, \quad * F_{A}+\mu(u)=\tau .
$$

Here $\tau \in Z(\mathfrak{g})$ is an element in the centre of the Lie algebra, $\mathcal{J}_{G}(M, \omega)$ denotes the space of G-invariant and $\omega$-tame almost complex structures on $M$, and $J: \Sigma \rightarrow \mathcal{J}_{\mathrm{G}}(M, \omega)$ is a smooth family of such almost complex structures. The space of such families of almost complex structures will be denoted by

$$
\mathcal{J}:=\mathcal{J}(\Sigma, M, \omega, \mu):=C^{\infty}\left(\Sigma, \mathcal{J}_{\mathrm{G}}(M, \omega)\right) .
$$

The covariant derivative of $u$ with respect to the connection $A$ is the 1 -form $d_{A} u \in \Omega^{1}\left(P, u^{*} T M\right)$ given by

$$
d_{A} u:=d u+X_{A}(u) .
$$

This 1-form is equivariant and horizontal and hence descends to a 1-form on $\Sigma$ with values in $u^{*} T M / \mathrm{G}$. The family of almost complex structures $J$ 
determines a G-invariant complex structure

$$
J_{u}(p):=J(\pi(p), u(p))
$$

on the bundle $u^{*} T M \rightarrow P$ and hence a complex structure on the bundle $u^{*} T M / \mathrm{G} \rightarrow \Sigma$, which will also be denoted by $J_{u}$. The term $\bar{\partial}_{J, A}(u)$ denotes the $(0,1)$-part of this 1 -form and so is a $(0,1)$-form on $\Sigma$ with values in $u^{*} T M /$ G. Its lift to a 1 -form on $P$ with values in $u^{*} T M$ will also be denoted by $\bar{\partial}_{J, A}(u)$ and is given by

$$
\bar{\partial}_{J, A}(u):=\frac{1}{2}\left(d_{A} u+J \circ d_{A} u \circ J_{\Sigma}\right) .
$$

The right hand side is well defined since $d_{A} u$ is horizontal. Namely, given a tangent vector $v \in T_{p} P$, lift the vector $J_{\Sigma} d \pi(p) v \in T_{\pi(p)} \Sigma$ to $T_{p} P$, and apply the linear map $J(\pi(p), u(p)) d_{A} u(p)$ to the lift. The resulting vector in $T_{u(p)} M$ is independent of the choice of the lift, because the 1-form $d_{A} u$ vanishes on vertical tangent vectors. Equations (1) were introduced in $[6$, $14,25]$. They are a generalized form of the vortex equations. In the case of linear actions on $\mathbb{C}^{n}$ they are known in the physics literature as gauged sigma models.

Remark 2.1. The space $C_{\mathrm{G}}^{\infty}(P, M) \times \mathcal{A}(P)$ is an infinite dimensional Fréchet manifold and admits a natural symplectic structure. The gauge group $\mathcal{G}(P)$ acts on this space by

$$
g^{*}(u, A)=\left(g^{-1} u, g^{-1} d g+g^{-1} A g\right)
$$

This action is Hamiltonian and the function

$$
C_{\mathrm{G}}^{\infty}(P, M) \times \mathcal{A}(P) \rightarrow C^{\infty}\left(\Sigma, \mathfrak{g}_{P}\right):(u, A) \mapsto * F_{A}+\mu(u)
$$

is a moment map for this action (see [6]). The space of solutions of (1) is invariant under the action of $\mathcal{G}(P)$. The quotient can be interpreted as a symplectic quotient whenever the space of pairs $(u, A)$ that satisfy $\bar{\partial}_{J, A}(u)=0$ is a symplectic submanifold of $C_{\mathrm{G}}^{\infty}(P, M) \times \mathcal{A}(P)$.

\subsection{Hamiltonian perturbations.}

Le $C_{\mathrm{G}}^{\infty}(M)$ be the space of smooth G-invariant functions on $M$. A Hamiltonian perturbation is a 1 -form $H \in \Omega^{1}\left(\Sigma, C_{\mathrm{G}}^{\infty}(M)\right)$. One can think of 
$H$ as a G-equivariant section of the vector bundle $T^{*} \Sigma \times M \rightarrow \Sigma \times M$. The space of Hamiltonian perturbations will be denoted by

$$
\mathcal{H}:=\mathcal{H}(\Sigma, M, \omega, \mu):=\Omega^{1}\left(\Sigma, C_{\mathrm{G}}^{\infty}(M)\right) .
$$

For $H \in \mathcal{H}$ and $\zeta \in T_{z} \Sigma$ we write $H_{\zeta}:=H_{z}(\zeta) \in C_{\mathrm{G}}^{\infty}(M)$ and denote by $X_{H_{\zeta}} \in \operatorname{Vect}(M, \omega)$ the G-invariant Hamiltonian vector field of $H_{\zeta}$, i.e.

$$
\iota\left(X_{H_{\zeta}}\right) \omega=d H_{\zeta}
$$

A Hamiltonian perturbation $H \in \mathcal{H}$ and a section $u \in C_{\mathrm{G}}^{\infty}(P, M)$ determine a 1 -form $X_{H}(u) \in \Omega^{1}\left(P, u^{*} T M\right)$ given by

$$
\left(X_{H}(u)\right)_{p}(v):=X_{H_{d \pi(p) v}}(u(p)) .
$$

This 1-form is equivariant and horizontal and so is

$$
d_{H, A}(u):=d_{A} u+X_{H}(u) .
$$

Hence $X_{H}(u)$ and $d_{H, A}(u)$ descend to 1 -forms on $\Sigma$ with values in $u^{*} T M / \mathrm{G}$. We denote

$$
\bar{\partial}_{J, H, A}(u):=\left(d_{H, A}(u)\right)^{0,1}=\bar{\partial}_{J, A}(u)+\left(X_{H}(u)\right)^{0,1} \in \Omega_{J_{u}}^{0,1}\left(\Sigma, u^{*} T M / \mathrm{G}\right)
$$

and replace (1) by the perturbed equations

$$
\bar{\partial}_{J, H, A}(u)=0, \quad * F_{A}+\mu(u)=\tau .
$$

\subsection{Energy.}

Fix a central element $\tau \in Z(\mathfrak{g})$, an almost complex structure $J \in$ $\mathcal{J}(\Sigma, M, \omega, \mu)$, and a perturbation $H \in \Omega^{1}\left(\Sigma, C_{\mathrm{G}}^{\infty}(M)\right)$. The energy of a pair $(u, A) \in C_{\mathrm{G}}^{\infty}(P, M) \times \mathcal{A}(P)$ is defined by

$$
E(u, A):=\frac{1}{2} \int_{\Sigma}\left(\left|d_{H, A}(u)\right|^{2}+\left|F_{A}\right|^{2}+|\mu(u)-\tau|^{2}\right) \mathrm{dvol}_{\Sigma} .
$$

This functional is invariant under the action of the gauge group $\mathcal{G}(P)$. Denote by $[\omega+\tau-\mu] \in H^{2}\left(M_{\mathrm{G}} ; \mathbb{R}\right)$ the equivariant cohomology class determined by the symplectic form $\omega$ and the moment map $\mu-\tau$ (see [6]), and denote by $[u] \in H_{2}\left(M_{\mathrm{G}} ; \mathbb{Z}\right)$ the homology class determined by $u$. More precisely, there is an equivariant classifying map $\theta: P \rightarrow$ EG and hence the map $(u, \theta): P \rightarrow M \times$ EG descends to a map

$$
u_{\mathrm{G}}: \Sigma \rightarrow M_{\mathrm{G}}:=M \times_{\mathrm{G}} \mathrm{EG} .
$$


The class $[u] \in H_{2}\left(M_{\mathrm{G}} ; \mathbb{Z}\right)$ is defined as the pushforward of the fundamental class $[\Sigma]$ under the map induced by $u_{\mathrm{G}}$. For every pair $(u, A) \in C_{\rho}^{\infty}(P, M) \times$ $\mathcal{A}(P)$ the cohomology pairing between the classes $[\omega+\tau-\mu]$ and $[u]$ is given by

$$
\langle[\omega+\tau-\mu],[u]\rangle=\int_{\Sigma}\left(u^{*} \omega-d\langle\mu(u)-\tau, A\rangle\right) .
$$

This topological invariant appears in the following energy identity. Another ingredient in this formula is the curvature of the Hamiltonian connection $H$. Since $\Sigma$ carries a volume form this curvature can be expressed as a function $\Omega_{H}: \Sigma \times M \rightarrow \mathbb{R}$ which is invariant under the G-action on $M$. It is defined by the formula

$$
\Omega_{H} \operatorname{dvol}_{\Sigma}:=d^{\Sigma} H+\frac{1}{2}\{H \wedge H\} \in \Omega^{2}\left(\Sigma, C_{\mathrm{G}}^{\infty}(M)\right),
$$

where $\{\cdot, \cdot\}$ denotes the Poisson bracket for functions on $M$. The Hofer norm of the curvature $\Omega_{H}$ is defined by

$$
\left\|\Omega_{H}\right\|:=\int_{\Sigma}\left(\sup _{x \in M} \Omega_{H}(z, x)-\inf _{x \in M} \Omega_{H}(z, x)\right) \operatorname{dvol}_{\Sigma}
$$

This quantity is independent of the volume form of $\Sigma$. The next proposition states the basic energy identity. The first term on the right is the $L^{2}$-norm of the terms in equation (3), the second term is a topological invariant, and the last term is bounded by $\left\|\Omega_{H}\right\|$.

Proposition 2.2. For every $A \in \mathcal{A}(P)$ and every $u \in C_{\mathrm{G}}^{\infty}(P, M)$,

$$
\begin{aligned}
E(u, A)= & \int_{\Sigma}\left(\left|\bar{\partial}_{J, H, A}(u)\right|^{2}+\frac{1}{2}\left|* F_{A}+\mu(u)-\tau\right|^{2}\right) \mathrm{dvol}_{\Sigma} \\
& +\langle[\omega+\tau-\mu],[u]\rangle+\int_{\Sigma} \Omega_{H}(u) \operatorname{dvol}_{\Sigma} .
\end{aligned}
$$

In particular, $E(u, A) \leq\langle[\omega+\tau-\mu],[u]\rangle+\left\|\Omega_{H}\right\|$ for every solution of (3).

Proof. Choose a holomorphic coordinate chart $\phi: U \rightarrow \Sigma$, where $U \subset \mathbb{C}$ is an open set, and let $\tilde{\phi}: U \rightarrow P$ be a lift of $\phi$, that is $\pi \circ \tilde{\phi}=\phi$. Then $u, A$, and $H$ are in local coordinates given by

$$
u^{\mathrm{loc}}:=u \circ \tilde{\phi}, \quad \tilde{\phi}^{*} A=\Phi d s+\Psi d t, \quad \phi^{*} H=F d s+G d t
$$


where $\Phi, \Psi: U \rightarrow \mathfrak{g}$ and $F, G: U \times M \rightarrow \mathbb{R}$. The pullback volume form on $U$ is dvoloc $=\lambda^{2} d s \wedge d t$ for some function $\lambda: U \rightarrow(0, \infty)$ and the metric is $\lambda^{2}\left(d s^{2}+d t^{2}\right)$. Hence

$$
\begin{aligned}
\phi^{*} \Omega_{H} & =\lambda^{-2}\left(\partial_{s} G-\partial_{t} F+\{F, G\}\right), \\
\tilde{\phi}^{*} F_{A} & =\left(\partial_{s} \Psi-\partial_{t} \Phi+[\Phi, \Psi]\right) d s \wedge d t, \\
\tilde{\phi}^{*} d_{A} u & =\left(\partial_{s} u^{\mathrm{loc}}+X_{\Phi}\left(u^{\mathrm{loc}}\right)\right) d s+\left(\partial_{t} u^{\mathrm{loc}}+X_{\Psi}\left(u^{\mathrm{loc}}\right)\right) d t, \\
\tilde{\phi}^{*} \bar{\partial}_{J, A}(u) & =\frac{1}{2}\left(\xi d s-J^{\mathrm{loc}}\left(s, t, u^{\mathrm{loc}}\right) \xi d t\right),
\end{aligned}
$$

where $\{F, G\}:=\omega\left(X_{F}, X_{G}\right)$ denotes the Poisson bracket on $M$,

$$
\xi:=\partial_{s} u^{\mathrm{loc}}+X_{\Phi}\left(u^{\mathrm{loc}}\right)+J^{\mathrm{loc}}\left(s, t, u^{\mathrm{loc}}\right)\left(\partial_{t} u^{\mathrm{loc}}+X_{\Psi}\left(u^{\mathrm{loc}}\right)\right),
$$

and $J^{\text {loc }}(s, t, x):=J(\phi(s, t), x)$ for $(s, t, x) \in U \times M$. In the following we shall drop the superscript "loc". Then (3) have the form

$$
\begin{array}{r}
\partial_{s} u+X_{\Phi}(u)+X_{F}(u)+J\left(\partial_{t} u+X_{\Psi}(u)+X_{G}(u)\right)=0, \\
\partial_{s} \Psi-\partial_{t} \Phi+[\Phi, \Psi]+\lambda^{2}(\mu(u)-\tau)=0,
\end{array}
$$

The pullback of the energy integrand under $\phi: U \rightarrow \Sigma$ is given by $e d s \wedge d t$ where $e: U \rightarrow \mathbb{R}$ is the function

$$
\begin{aligned}
e:= & \frac{1}{2}\left|\partial_{s} u+X_{\Phi}(u)+X_{F}(u)\right|^{2}+\frac{1}{2}\left|\partial_{t} u+X_{\Psi}(u)+X_{G}(u)\right|^{2} \\
& +\frac{1}{2 \lambda^{2}}\left|\partial_{s} \Psi-\partial_{t} \Phi+[\Phi, \Psi]\right|^{2}+\frac{\lambda^{2}}{2}|\mu(u)-\tau|^{2} \\
= & \frac{1}{2}\left|\partial_{s} u+X_{\Phi}(u)+X_{F}(u)+J\left(\partial_{t} u+X_{\Psi}(u)+X_{G}(u)\right)\right|^{2} \\
& +\frac{\lambda^{2}}{2}\left|\lambda^{-2}\left(\partial_{s} \Psi-\partial_{t} \Phi+[\Phi, \Psi]\right)+\mu(u)-\tau\right|^{2}+R .
\end{aligned}
$$

The remainder term $R$ has the form

$$
\begin{aligned}
R:= & \omega\left(\partial_{s} u+X_{\Phi}(u)+X_{F}(u), \partial_{t} u+X_{\Psi}(u)+X_{G}(u)\right) \\
& -\left\langle\partial_{s} \Psi-\partial_{t} \Phi+[\Phi, \Psi], \mu(u)-\tau\right\rangle \\
= & \omega\left(\partial_{s} u, \partial_{t} u\right)-\partial_{s}(G(u)+\langle\mu(u)-\tau, \Psi\rangle)+\partial_{t}(F(u)+\langle\mu(u)-\tau, \Phi\rangle) \\
& +\left(\partial_{s} G-\partial_{t} F+\{F, G\}\right) \circ u .
\end{aligned}
$$

This proves (4). 


\subsection{Unique continuation.}

A solution $(u, A)$ of $(3)$ is called horizontal if $d_{H, A}(u) \equiv 0$ and $\mu(u) \equiv \tau$.

Lemma 2.3. Let $(u, A)$ be a solution of (3) with $H=0$. Then $(u, A)$ is horizontal if and only if the homology class $[u] \in H_{2}\left(M_{\mathrm{G}} ; \mathbb{Z}\right)$ is torsion.

Proof. The "if" part follows from the energy identity. To prove the converse note that $A$ is flat for every horizontal solution $(u, A)$ of $(3)$ and hence, in the case $H=0$, every equivariant cohomology class vanishes on $[u]$ (see [6]).

Lemma 2.4. Suppose $u$ and $A$ satisfy (3) with $H=0$. If $d_{A} u$ and $\mu(u)-\tau$ vanish to infinite order at some point $p_{0} \in P$ then $(u, A)$ is horizontal.

Proof. Replacing $\mu$ by $\mu-\tau$ we may assume that $\tau=0$. Consider the equations in their local coordinate form (5). In the case $H=0$ they read

$$
v_{s}+J v_{t}=0, \quad \kappa+\lambda^{2} \mu(u)=0
$$

for $u: U \rightarrow M$ and $\Phi, \Psi: U \rightarrow \mathfrak{g}$, where $v_{s}, v_{t}: U \rightarrow u^{*} T M$ and $\kappa: U \rightarrow \mathfrak{g}$ are defined by

$$
v_{s}:=\partial_{s} u+L_{u} \Phi, \quad v_{t}:=\partial_{t} u+L_{u} \Psi, \quad \kappa:=\partial_{s} \Psi-\partial_{t} \Phi+[\Phi, \Psi],
$$

where $L_{x}: \mathfrak{g} \rightarrow T_{x} M$ denotes the infinitesimal action given by $L_{x} \eta:=X_{\eta}(x)$. Let us denote

$$
\begin{array}{llll}
\nabla_{A, s} \xi & :=\nabla_{s} \xi+\nabla_{\xi} X_{\Phi}(u), & \nabla_{A, t} \xi & :=\nabla_{t} \xi+\nabla_{\xi} X_{\Psi}(u), \\
\nabla_{A, s} \eta & :=\partial_{s} \eta+[\Phi, \eta], & \nabla_{A, t} \eta & :=\partial_{t} \eta+[\Psi, \eta],
\end{array}
$$

for $\xi: U \rightarrow u^{*} T M$ and $\eta: U \rightarrow \mathfrak{g}$. Then

$$
\nabla_{A, s} L_{u} \eta-L_{u} \nabla_{A, s} \eta=\nabla_{v_{s}} X_{\eta}(u), \quad \nabla_{A, t} L_{u} \eta-L_{u} \nabla_{A, t} \eta=\nabla_{v_{t}} X_{\eta}(u) .
$$

Since $d \mu(u)=-L_{u}^{*} J$ we have

$$
\nabla_{A, s} \mu(u)=d \mu(u) v_{s}=-L_{u}^{*} v_{t}, \quad \nabla_{A, t} \mu(u)=d \mu(u) v_{t}=L_{u}^{*} v_{s} .
$$


It follows from (6), (7), and (8) that

$$
\nabla_{A, t}\left(\nabla_{A, t} v_{s}-\nabla_{A, s} v_{t}\right)=\left(\partial_{t} \lambda^{2}\right) L_{u} \mu(u)+\lambda^{2} d \mu(u) v_{t}+\lambda^{2} \nabla_{v_{t}} X_{\mu(u)}(u) .
$$

Since $0=\left(\mathcal{L}_{X_{\eta}} J\right) \xi=\left(\nabla_{X_{\eta}} J\right) \xi+J \nabla_{\xi} X_{\eta}-\nabla_{J \xi} X_{\eta}$, we obtain

$$
\nabla_{A, s} J=\nabla_{v_{s}} J+\partial_{s} J, \quad \nabla_{A, t} J=\nabla_{v_{t}} J+\partial_{t} J,
$$

and hence, using the identity $\left(\nabla_{v_{s}} J\right) v_{t}=\left(\nabla_{v_{t}} J\right) v_{s}$,

$$
\begin{aligned}
\nabla_{A, s} v_{s}+\nabla_{A, t} v_{t} & =\nabla_{A, t}\left(J v_{s}\right)-\nabla_{A, s}\left(J v_{t}\right) \\
& =\left(\partial_{t} J\right) v_{s}-\left(\partial_{s} J\right) v_{t}+\lambda^{2} J L_{u} \mu(u) .
\end{aligned}
$$

This gives rise to an inequality of the form

$$
\left|\nabla_{A, s}\left(\nabla_{A, s} v_{s}+\nabla_{A, t} v_{t}\right)\right| \leq c_{1}\left(\left|v_{s}\right|+\left|\partial_{s} v_{s}\right|+\left|\partial_{t} v_{s}\right|+|\mu(u)|\right) .
$$

Moreover, by (9),

$$
\left|\nabla_{A, t}\left(\nabla_{A, t} v_{s}-\nabla_{A, s} v_{t}\right)\right| \leq c_{2}\left(\left|v_{s}\right|+|\mu(u)|\right)
$$

and, by the curvature identity $\nabla_{A, s} \nabla_{A, t} \xi-\nabla_{A, t} \nabla_{A, s} \xi=R\left(v_{s}, v_{t}\right) \xi+\nabla_{\xi} X_{\kappa}(u)$,

$$
\left|\nabla_{A, s} \nabla_{A, t} v_{t}-\nabla_{A, t} \nabla_{A, s} v_{t}\right| \leq c_{3}\left|v_{s}\right|
$$

Putting these three inequalities together we obtain

$$
\left|\nabla_{A, s} \nabla_{A, s} v_{s}+\nabla_{A, t} \nabla_{A, t} v_{s}\right| \leq c_{4}\left(\left|v_{s}\right|+\left|\partial_{s} v_{s}\right|+\left|\partial_{t} v_{s}\right|+|\mu(u)|\right) .
$$

Moreover,

$$
\nabla_{A, s}\left(L_{u}^{*} \xi\right)-L_{u}^{*} \nabla_{A, s} \xi=\rho\left(v_{s}, \xi\right), \quad \nabla_{A, t}\left(L_{u}^{*} \xi\right)-L_{u}^{*} \nabla_{A, t} \xi=\rho\left(v_{t}, \xi\right)
$$

where $\rho \in \Omega^{2}(M, \mathfrak{g})$ is defined by $\left\langle\eta, \rho\left(\xi_{1}, \xi_{2}\right)\right\rangle:=\left\langle\nabla_{\xi_{1}} X_{\eta}, \xi_{2}\right\rangle$ (see [15]). Hence, by (8) and (6),

$$
\nabla_{A, s} \nabla_{A, s} \mu(u)+\nabla_{A, t} \nabla_{A, t} \mu(u)=\lambda^{2} L_{u}^{*} L_{u} \mu(u)-2 \rho\left(v_{s}, v_{t}\right) .
$$

Hence there exists a constant $c$ such that

$$
\left|\Delta v_{s}\right| \leq c\left(\left|v_{s}\right|+\left|\partial_{s} v_{s}\right|+\left|\partial_{t} v_{s}\right|+|\mu(u)|\right), \quad|\Delta \mu(u)| \leq c\left(|\mu(u)|+\left|v_{s}\right|\right) .
$$

Hence it follows from Aronszajn's theorem that, if $v_{s}$ and $\mu(u)$ vanish to infinite order at a point in $U$ and $U$ is connected, then $v_{s}$ and $\mu(u)$ vanish identically on $U$. 
Lemma 2.5. Suppose $u$ and $A$ satisfy (3) with $H=0$. If there exists an open set $U \subset P$ such that $d_{A} u(p)=0$ and $L_{u(p)}: \mathfrak{g} \rightarrow T_{u(p)} M$ is injective for every $p \in U$ then $(u, A)$ is horizontal.

Proof. By (6), $L_{u}(\mu(u)-\tau)=0$ in $U$. Hence $\mu(u)=\tau$ in $U$ and hence, by Lemma 2.4, we have $d_{A} u \equiv 0$ and $\mu(u) \equiv \tau$.

\subsection{Convexity.}

Definition 2.6. A convex structure on $(M, \omega, \mu)$ is a pair $(f, J)$ where $J \in \mathcal{J}(M, \omega)$ is a G-invariant $\omega$-compatible almost complex structure on $M$ and $f: M \rightarrow[0, \infty)$ is a smooth function satisfying the following conditions.

(C1) $f$ is G-invariant and proper.

(C2) There exists a constant $c_{0}>0$ such that

$$
f(x) \geq c_{0} \quad \Longrightarrow \quad\left\langle\nabla_{\xi} \nabla f(x), \xi\right\rangle+\left\langle\nabla_{J \xi} \nabla f(x), J \xi\right\rangle \geq 0
$$

for every $x \in M$ and every $\xi \in T_{x} M$. Here $\nabla$ denotes the Levi-Civita connection of the metric $\langle\cdot, \cdot\rangle=\omega(\cdot, J \cdot)$.

(C3) There exists a constant $c_{0}>0$ such that

$$
f(x) \geq c_{0} \quad \Longrightarrow \quad d f(x) J(x) L_{x} \mu(x) \geq 0
$$

for every $x \in M$.

The second hypothesis says that the upward gradient flow of $f$ expands the metric outside of a sufficiently large compact set. It is sometimes useful to assume condition $(C 3)$ for all moment maps $\mu-\tau$.

$\left(\mathbf{C 3}^{\prime}\right)$ There exists a continuous function $Z(\mathfrak{g}) \rightarrow \mathbb{R}: \tau \mapsto c_{0}(\tau)$ such that

$$
f(x) \geq c_{0}(\tau) \quad \Longrightarrow \quad d f(x) J(x) L_{x}(\mu(x)-\tau) \geq 0
$$

for every $\tau \in Z(\mathfrak{g})$ and every $x \in M$.

A convex structure $(f, J)$ that satisfies $\left(C 3^{\prime}\right)$ is called strongly convex.

Lemma 2.7. Fix a homology class $B \in H_{2}\left(M_{\mathrm{G}} ; \mathbb{Z}\right)$ and let $\left(f, J_{0}\right)$ be a convex structure for $(M, \omega, \mu-\tau)$. Denote

$$
M_{0}:=\left\{x \in M \mid f(x) \leq c_{0}\right\}
$$


where $c_{0}=c_{0}(\tau)$ is chosen such that $(C 2)$ and $\left(C 3^{\prime}\right)$ are satisfied and

$$
f(x)>c_{0} \quad \Longrightarrow \quad|\mu(x)-\tau|^{2}>\frac{\langle[\omega+\tau-\mu], B\rangle}{\operatorname{Vol}(\Sigma)}
$$

Let $P$ be a principal G-bundle over a compact Riemann surface $\Sigma$, suppose that $J \in \mathcal{J}$ agrees with $J_{0}$ outside $M_{0}$, and let $H \in \mathcal{H}$ be a Hamiltonian perturbation with support in $M_{0}$. Then every solution $(u, A)$ of $(3)$ representing the class $B$ satisfies $u(P) \subset M_{0}$.

Proof. Assume without loss of generality that $\tau=0$ and continue the notation of the proof of Lemma 2.4. Since $\nabla f$ is a G-invariant vector field we have $\left[\nabla f, X_{\eta}\right]=0$ for every $\eta \in \mathfrak{g}$ and hence

$$
\nabla_{A, s} \nabla f(u)=\nabla_{v_{s}} \nabla f(u), \quad \nabla_{A, t} \nabla f(u)=\nabla_{v_{t}} \nabla f(u) .
$$

Let $\Delta:=\partial_{s}^{2}+\partial_{t}^{2}$ denote the standard Laplacian. If $f(u)>c_{0}$ then

$$
\begin{aligned}
\Delta f(u) & =\partial_{s}\left\langle\nabla f(u), v_{s}\right\rangle+\partial_{t}\left\langle\nabla f(u), v_{t}\right\rangle \\
& =\left\langle\nabla_{A, s} \nabla f(u), v_{s}\right\rangle+\left\langle\nabla_{A, t} \nabla f(u), v_{t}\right\rangle+\left\langle\nabla f(u), \nabla_{A, s} v_{s}+\nabla_{A, t} v_{t}\right\rangle \\
& =\left\langle\nabla_{v_{s}} \nabla f(u), v_{s}\right\rangle+\left\langle\nabla_{v_{t}} \nabla f(u), v_{t}\right\rangle+\lambda^{2}\left\langle\nabla f(u), J L_{u} \mu(u)\right\rangle \\
& \geq\left\langle\nabla_{v_{s}} \nabla f(u), v_{s}\right\rangle+\left\langle\nabla_{v_{t}} \nabla f(u), v_{t}\right\rangle .
\end{aligned}
$$

Here the third equality follows from (10) and the last inequality from $(C 3)$. Now suppose, by contradiction, that $m:=\max _{P} f \circ u>c_{0}$ and choose a local coordinate chart as above near a point where $f \circ u$ attains its maximum. Since $f \circ u$ is subharmonic it follows from the mean value inequality that $f \circ u=m$ in a neighbourhood of this maximum. Hence the subset of $P$ where $f \circ u=m$ is open and closed, and hence $f \circ u \equiv m$. Hence, by (11),

$$
E(u, A) \geq \int_{\Sigma}|\mu(u)|^{2} \operatorname{dvol}_{\Sigma} \geq \operatorname{Vol}(\Sigma) \inf _{P}|\mu \circ u|^{2}>\langle[\omega-\mu], B\rangle .
$$

Since $\Omega_{H}$ vanishes on the image of $u$, this contradicts the energy identity.

Example 2.8 ([6]). Consider the linear action of a compact Lie group $\mathrm{G}$ on $\mathbb{C}^{n}$ by a homomorphism $\rho: \mathrm{G} \rightarrow \mathrm{U}(n)$ with proper moment map $\mu_{\rho}: \mathbb{C}^{n} \rightarrow \mathfrak{g}$ given by

$$
\mu_{\rho}(x):=\pi_{\rho}\left(-\frac{i}{2} x x^{*}\right)
$$


where $\pi_{\rho}:=\dot{\rho}^{*}: \mathfrak{u}(n) \rightarrow \mathfrak{g}$ is the dual operator of the Lie algebra homomorphism $\dot{\rho}: \mathfrak{g} \rightarrow \mathfrak{u}(n)$ with respect to the inner product $\langle A, B\rangle:=\operatorname{trace}\left(A^{*} B\right)$ on $\mathfrak{u}(n)$. A strongly convex structure for $\rho$ is the pair $(f, J)$ with $J=i$ and

$$
f(x)=\frac{1}{2}|x|^{2}
$$

To see this note that $\nabla f(x)=x$ and

$$
d f(x) J L_{x}\left(\mu_{\rho}(x)-\tau\right)=\left\langle\mu_{\rho}(x), \mu_{\rho}(x)-\tau\right\rangle
$$

Note that the pair $(f, i)$ with $f(x)=|\mu(x)|^{2} / 2$ need not be a convex structure. An example is the action of $\mathbb{T}^{2}$ on $\mathbb{C}^{2}$ by $\left(t_{1}, t_{2}\right) \cdot\left(x_{1}, x_{2}\right)=\left(t_{1} x_{1}, t_{2} x_{2}\right)$.

Example 2.9 (Contact boundaries). Suppose $(M, \omega)$ is a compact symplectic manifold with boundary $\partial M$, equipped with a Hamiltonian G-action generated by a moment map $\mu: M \rightarrow \mathbb{R}$. Suppose that $X \in \operatorname{Vect}(M)$ is a G-invariant vector field which points out on the boundary and satisfies

$$
\mathcal{L}_{X} \omega=\omega, \quad \omega\left(X_{\mu}, X\right) \geq 0
$$

near $\partial M$. Such a vector field gives rise to a convex structure as follows. Let $\phi_{t}$ denote the flow of $X$ and choose a G-invariant $\omega$-compatible almost complex structure $J$ on $M$ such that

$$
d \phi_{t}(x) J(x)=J\left(\phi_{t}(x)\right) d \phi_{t}(x), \quad \omega(X(x), J(x) X(x))=1,
$$

and $\omega(X(x), J(x) v)=0$ for $x \in \partial M, v \in T_{x} \partial M$, and $-\varepsilon<t \leq 0$. Then the function $f: M \rightarrow \mathbb{R}$, defined by

$$
f\left(\phi_{t}(x)\right):=t
$$

for $x \in \partial M$ and $-\varepsilon<t \leq 0$ defines a convex structure near $\partial M$. Its gradient is the vector field $X$ and its covariant Hessian is half the identity. Moreover, the manifold can be extended by attaching a cylindrical end of the form $\partial M \times[0, \infty)$ with the obvious extensions of the symplectic and almost complex structures to obtain a noncompact manifold as above.

Example 2.10 (Convex fibrations). Let $\mathrm{G}$ and $\mathrm{H}$ be compact connected Lie groups with Lie angebras $\mathfrak{g}=\operatorname{Lie}(\mathrm{G})$ and $\mathfrak{h}=\operatorname{Lie}(\mathrm{H})$. Let $(M, \omega)$ be a (not necessarily compact) symplectic manifold equipped with Hamiltonian action by both Lie groups $\mathrm{G}$ and $\mathrm{H}$, generated by moment maps $\mu_{\mathrm{G}}: N \rightarrow \mathfrak{g}$ 
and $\mu_{\mathrm{H}}: N \rightarrow \mathfrak{h}$. We assume throughout that the action of $\mathrm{G}$ commutes with the action of $\mathrm{H}$. This is equivalent to the condition

$$
\mu_{\mathrm{G}}(h x)=\mu_{\mathrm{G}}(x), \quad \mu_{\mathrm{H}}(g x)=\mu_{\mathrm{H}}(x)
$$

for $g \in \mathrm{G}, h \in \mathrm{H}$, and $x \in M$. Let $(S, \sigma)$ be a compact symplectic manifold and $\pi_{S}: Q \rightarrow S$ be a principal H-bundle. We assume that $Q$ is equipped with a connection $B \in \mathcal{A}(Q) \subset \Omega^{1}(Q, \mathfrak{h})$ with nonpositive curvature. This means that there exists a $\sigma$-compatible almost complex structure $J_{S} \in \mathcal{J}(S, \sigma)$ such that

$$
d \pi(q) w^{\prime}=J_{S} d \pi(q) w \quad \Longrightarrow \quad\left\langle F_{B}\left(w, w^{\prime}\right), \mu_{\mathrm{H}}(x)\right\rangle \leq 0 .
$$

Then the manifold

$$
\tilde{M}:=Q \times_{\mathrm{H}} M
$$

carries a symplectic form $\tilde{\omega} \in \Omega^{2}(\tilde{M})$ whose pullback under the projection $\pi: Q \times M \rightarrow \tilde{M}$ is given by

$$
\pi^{*} \tilde{\omega}:=\pi_{2}^{*} \omega-d\left\langle B, \mu_{\mathrm{H}}\right\rangle+\pi_{1}^{*} \sigma
$$

where $\pi_{1}: Q \times M \rightarrow S$ and $\pi_{2}: Q \times M \rightarrow M$ denote the obvious projections. A moment map $\tilde{\mu}: \tilde{M} \rightarrow \mathfrak{g}$ for the obvious G-action on $\tilde{M}$ is given by

$$
\tilde{\mu}([q, x]):=\mu_{\mathrm{G}}(x)
$$

for $q \in Q$ and $x \in M$. Here $[q, x]=\left[q h, h^{-1} x\right]$ denotes the equivalence class of the pair $(q, x)$ in $Q \times_{\mathrm{H}} M$. Note that if $\mu_{\mathrm{G}}$ is proper then so is $\tilde{\mu}$. Note also that every $\mathrm{H}$-invariant and $\omega$-compatible almost complex structure $J \in \mathcal{J}_{\mathrm{H}}(M, \omega)$ induces an almost complex $\tilde{J} \in \mathcal{J}(\tilde{M}, \tilde{\omega})$ which acts by $J_{S}$ on the horizontal subbundle and by $J$ on the vertical subbundle of $T \tilde{M}$. If $J$ is invariant under both $\mathrm{G}$ and $\mathrm{H}$, then $\tilde{J}$ in invariant under the G-action on $\tilde{M}$.

Now suppose that $(J, f)$ is a convex structure for the G-action on $M$ (as in Definition 2.6). Suppose also that $J$ and $f$ are H-invariant. Then the above almost complex structure $\tilde{J}$ and the function $\tilde{f}: \tilde{M} \rightarrow[0, \infty)$ given by

$$
\tilde{f}([q, x]):=f(x)
$$

define a convex structure for the G-action on $\tilde{M}$. To see this, note that the gradient $\nabla \tilde{f}$ is given by $\nabla \tilde{f}([q, x])=[0, \nabla f(x)]$. Let $\phi_{t}: M \rightarrow M$ denote the (upward) gradient flow of $f$. Then the gradient flow of $\tilde{f}$ is given by

$$
\tilde{\phi}_{t}([q, x])=\left[q, \phi_{t}(x)\right] .
$$


for $q \in Q$ and $x \in M$. Hence $d \tilde{\phi}_{t}([q, x])[w, \xi]=\left[w, d \phi_{t}(x) \xi\right]$. In particular, the image of a horizontal tangent vector $\tilde{\xi}_{0}:=\left[w,-Y_{B_{q}(w)}(x)\right]$ under $d \tilde{\phi}_{t}([q, x])$ is the horizontal vector

$$
\tilde{\xi}_{t}:=\left[w,-Y_{B_{q}(w)}\left(\phi_{t}(x)\right)\right] \in T_{\left[q, \phi_{t}(x)\right]} \tilde{M}
$$

and so

$$
\left|\tilde{\xi}_{t}\right|^{2}=\left|d \pi_{S}(q) w\right|^{2}+\left|d \phi_{t}(x) Y_{B_{q}(w)}\right|^{2}-\left\langle F_{B}\left(w, w^{\prime}\right), \mu_{\mathrm{H}}(x)\right\rangle,
$$

where $w^{\prime} \in T_{q} Q$ satisfies $d \pi_{S}(q) w^{\prime}=J_{S} d \pi_{S}(q) w$. Here we have used the fact that $\phi_{t}$ commutes with the action of $\mathrm{H}$ and so $\mu_{\mathrm{H}} \circ \phi_{t}=\mu_{\mathrm{H}}$. It follows from the hypotheses on $(J, f)$ that the function $t \mapsto\left|\tilde{\xi}_{t}\right|$ is nondecreasing whenever $\tilde{f}\left(\left[q, \phi_{t}(x)\right]\right)$ is sufficiently large.

\section{Compactness and Regularity.}

\subsection{Regularity.}

The next theorem asserts that every weak solution of equations (3) is gauge equivalent to a strong solution. For an integer $\ell \geq 1$ we denote by

$$
\mathcal{J}^{\ell}=\mathcal{J}^{\ell}(\Sigma, M, \omega, \mu)
$$

the space of almost complex structures of class $C^{\ell}$ and by

$$
\mathcal{H}^{\ell}=\mathcal{H}^{\ell}(\Sigma, M, \omega, \mu)
$$

the space of Hamiltonian perturbations of class $C^{\ell}$ (see Section 2.2). Thus $\mathcal{H}^{\ell}$ is the vector space of G-equivariant $\mathbb{C}^{\ell}$-sections of the vector bundle $T^{*} \Sigma \times M \rightarrow \Sigma \times M$. For $\ell=\infty$ we write $\mathcal{J}^{\infty}=: \mathcal{J}$ and $\mathcal{H}^{\infty}=: \mathcal{H}$. Consider the symplectic fibre bundle

$$
\tilde{M}:=P \times_{\mathrm{G}} M \rightarrow \Sigma
$$

with fibres diffeomorphic to $M$. There is a one-to-one correspondence between sections $\tilde{u}: \Sigma \rightarrow \tilde{M}$ and G-equivariant functions $u: P \rightarrow M$ via

$$
\tilde{u} \circ \pi(p)=[p, u(p)]
$$

for $p \in P$. For a positive integer $k$ and a constant $p>2$ we denote by $W_{\mathrm{G}}^{k, p}(P, M)$ the Banach manifold of all continuous G-equivariant functions $u: P \rightarrow M$ such that the corresponding section $\tilde{u}: \Sigma \rightarrow \tilde{M}$ is of class $W^{k, p}$. 
Theorem 3.1. Fix a constant $p>2$ and let $\ell$ be either a positive integer or be equal to $\infty$. Let $J \in \mathcal{J}^{\ell}$ and $H \in \mathcal{H}^{\ell+1}$. If $u \in W_{\mathrm{G}}^{1, p}(P, M)$ and $A \in \mathcal{A}^{1, p}(P)$ satisfy (3) then there exists a gauge transformation $g \in \mathcal{G}^{2, p}(P)$ such that $g^{-1} u$ and $g^{*} A$ are of class $W^{\ell+1, p}$. For $\ell=\infty$ this means that $g^{-1} u$ and $g^{*} A$ are smooth.

Proof. Let $(u, A) \in W^{1, p}\left(\Sigma, P \times_{\mathrm{G}} M\right) \times \mathcal{A}^{1, p}(P)$ be a solution of (3). Assume first that there exists a smooth connection $A_{0} \in \mathcal{A}(P)$ such that

$$
d_{A_{0}}^{*}\left(A-A_{0}\right)=0 \text {. }
$$

Under this assumption we shall prove that the pair $(u, A)$ is of class $W^{\ell+1, p}$. Denote $\alpha:=A-A_{0} \in W^{1, p}\left(\Sigma, T^{*} \Sigma \otimes \mathfrak{g}_{P}\right)$. Then the first equation in (3) has the form

$$
\bar{\partial}_{J, A_{0}}(u)=-\left(X_{\alpha}(u)+X_{H}(u)\right)^{0,1},
$$

where the $(0,1)$-part of the 1 -form $X_{\alpha}(u)-X_{H}(u)$ on $\Sigma$ with values in $u^{*} T M / \mathrm{G}$ is understood with respect to $J_{u}$. The second equation in (3) and (13) together have the form

$$
d_{A_{0}} \alpha=-F_{A_{0}}-\frac{1}{2}[\alpha \wedge \alpha]+(\tau-\mu(u)) \operatorname{dvol}_{\Sigma}, \quad d_{A_{0}}^{*} \alpha=0 .
$$

We prove by induction that $u$ and $A$ are of class $W^{k, p}$ for every integer $k \leq \ell+1$. For $k=1$ this holds by assumption. If $u$ and $A$ are of class $W^{k, p}$ for some $k \leq \ell$ then, by (15), $d_{A_{0}} \alpha$ and $d_{A_{0}}^{*} \alpha$ are of class $W^{k, p}$ and hence $\alpha$ is of class $W^{k+1, p}$. Moreover, by $(14), \bar{\partial}_{J, A_{0}}(u)$ is of class $W^{k, p}$ and the complex structure $J_{u}$ on the bundle $u^{*} T M / \mathrm{G}$ is also of class $W^{k, p}$. Hence $u$ is of class $W^{k+1, p}$ (see [23, Proposition B.4.7]). This completes the induction. Hence the pair $(u, A)$ is of class $W^{\ell+1, p}$, and is smooth in the case $\ell=\infty$.

Thus we have proved the theorem under the assumption that $A$ satisfies (13) for some smooth connection $A_{0}$. In general, it follows from the local slice theorem (see Theorem B.1) that there exists a smooth connection $A_{0}$ and a gauge transformation $g \in \mathcal{G}^{2, p}(P)$ such that

$$
d_{A}^{*}\left(g_{*} A_{0}-A\right)=0 .
$$

Then $g^{*} A$ satisfies (13) and hence the pair $\left(g^{-1} u, g^{*} A\right)$ is of class $W^{\ell+1, p}$, and is smooth in the case $\ell=\infty$. 


\subsection{Compactness with bounded derivatives.}

In this section we prove a compactness result for solutions of (3) with values in a fixed compact subset of $M$ under the hypothesis that the first derivatives satisfy a uniform $L^{\infty}$-bound. We assume that $\omega_{\nu} \in \Omega^{2}(M)$ is a sequence of symplectic forms on $M$ converging uniformly with all derivatives to a symplectic form $\omega$ and that $\mu_{\nu}: M \rightarrow \mathfrak{g}$ is a sequence of moment maps (corresponding to a sequence of $\omega_{\nu}$-Hamiltonian G-actions on $M$ ) that converges uniformly with all derivatives to the moment map $\mu$. We assume that $\omega_{\nu}$ agrees with $\omega$ and $\mu_{\nu}$ agrees with $\mu$ outside of a compact set. We assume further that $\operatorname{dvol}_{\Sigma, \nu}$ is a sequence of volume forms on $\Sigma$ converging in the $C^{\infty}$-topology to $\operatorname{dvol}_{\Sigma}$ and $J_{\Sigma, \nu}$ is a sequence of complex structures on $\Sigma$ converging in the $C^{\infty}$-topology to $J_{\Sigma}$.

Theorem 3.2. Let $\ell$ be either a positive integer or be equal to $\infty$. Suppose that

$$
\left(J_{\nu}, H_{\nu}\right) \in \mathcal{J}^{\ell}\left(\Sigma, M, \omega_{\nu}, \mu_{\nu}\right) \times \mathcal{H}^{\ell+1}\left(\Sigma, M, \omega_{\nu}, \mu_{\nu}\right)
$$

is a sequence such that $J_{\nu}$ converges to $J \in \mathcal{J}^{\ell}(\Sigma, M, \omega, \mu)$ in the $C^{\ell}$-norm on every compact set, and that $H_{\nu}$ converges to $H \in \mathcal{H}^{\ell+1}(\Sigma, M, \omega, \mu)$ in the $C^{\ell+1}$-norm on every compact set. Suppose further that $\tau_{\nu} \in Z(\mathfrak{g})$ converges to $\tau$. For every $\nu$ let $\left(u_{\nu}, A_{\nu}\right) \in W_{\mathrm{G}}^{1, p}(P, M) \times \mathcal{A}^{1, p}(P)$ be a solution of (3) with $\left(\mu, J_{\Sigma}, \operatorname{dvol}_{\Sigma}, J, H, \tau\right)$ replaced by $\left(\mu_{\nu}, J_{\Sigma, \nu}, \operatorname{dvol}_{\Sigma, \nu}, J_{\nu}, H_{\nu}, \tau_{\nu}\right)$. Suppose that there exist a constant $c>0$ and a compact set $K \subset M$ such that

$$
u_{\nu}(P) \subset K, \quad\left\|d_{A_{\nu}} u_{\nu}\right\|_{L^{\infty}} \leq c
$$

for every $\nu$. Then there exists a sequence of gauge transformations $g_{\nu} \in$ $\mathcal{G}^{2, p}(P)$ such that the sequence $\left(g_{\nu}^{-1} u_{\nu}, g_{\nu}^{*} A_{\nu}\right)$ has a $C^{\ell}$-convergent subsequence.

Lemma 3.3. Fix positive integers $k$ and $n$, a real number $p>2$, an open set $U \subset \mathbb{C}$, and a compact subset $K \subset U$. Let $\mathcal{J}_{n} \subset \mathbb{R}^{2 n \times 2 n}$ denote the set of complex structures on $\mathbb{R}^{2 n}$. Then for every constant $c_{0}>0$ there exists a constant $c=c\left(c_{0}, K, U, n, k, p\right)>0$ such that the following holds. If $J \in W^{k, p}\left(U, \mathcal{J}_{n}\right)$ satisfies

$$
\|J\|_{W^{k, p}(U)} \leq c_{0}
$$

then every function $u \in W^{k+1, p}\left(U, \mathbb{R}^{2 n}\right)$ satisfies the inequality

$$
\|u\|_{W^{k+1, p}(K)} \leq c\left(\left\|\partial_{s} u+J \partial_{t} u\right\|_{W^{k, p}(U)}+\|u\|_{W^{k, p}(U)}+\|u\|_{W^{1, \infty}(U)}\right) .
$$


Proof. We argue by contradiction. Suppose that there exist sequences $J_{\nu} \in$ $W^{k, p}\left(U, \mathcal{J}_{n}\right)$ and $u_{\nu} \in W^{k+1, p}\left(U, \mathbb{R}^{2 n}\right)$ such that

$$
\left\|J_{\nu}\right\|_{W^{k, p}(U)} \leq c_{0}, \quad\left\|u_{\nu}\right\|_{W^{k+1, p}(K)} \rightarrow \infty
$$

and

$$
\left\|\partial_{s} u_{\nu}+J_{\nu} \partial_{t} u_{\nu}\right\|_{W^{k, p}(U)}+\left\|u_{\nu}\right\|_{W^{k, p}(U)}+\left\|u_{\nu}\right\|_{W^{1, \infty}(U)} \leq 1 .
$$

Passing to a subsequence, if necessary, we may assume that there is a complex structure $J_{0} \in W^{k, p}\left(U, \mathcal{J}_{n}\right)$ such that $J_{\nu}$ converges to $J_{0}$ in the weak $W^{k, p}$-topology and in the strong $C^{0}$-topology. Choose a smooth cutoff function $\beta: U \rightarrow[0,1]$ with compact support such that $\left.\beta\right|_{K} \equiv 1$ and define

$$
v_{\nu}:=\beta u_{\nu}
$$

Then $v_{\nu}$ is bounded in $W^{k, p}$ and $W^{1, \infty}$ and it satisfies the identity

$$
\partial_{s} v_{\nu}+J_{0} \partial_{t} v_{\nu}=\beta\left(\partial_{s} u_{\nu}+J_{\nu} \partial_{t} u_{\nu}\right)+\left(\partial_{s} \beta\right) u_{\nu}+\left(\partial_{t} \beta\right) J_{\nu} u_{\nu}+\left(J_{0}-J_{\nu}\right) \partial_{t} v_{\nu}
$$

The elliptic estimate for the operator $\partial_{s}+J_{0} \partial_{t}$ has the form

$$
\|v\|_{W^{k+1, p}(U)} \leq c\left(\left\|\partial_{s} v+J_{0} \partial_{t} v\right\|_{W^{k, p}(U)}+\|v\|_{W^{k, p}(U)}\right)
$$

for some constant $c>0$ and every function $v: U \rightarrow \mathbb{R}^{2 n}$ with compact support (see for example [23, Proposition B.4.7]). Hence

$$
\begin{aligned}
\left\|v_{\nu}\right\|_{W^{k+1, p}(U)} \leq & c\left(\left\|\beta\left(\partial_{s} u_{\nu}+J_{\nu} \partial_{t} u_{\nu}\right)\right\|_{W^{k, p}(U)}\right. \\
& +\left\|\left(\partial_{s} \beta\right) u_{\nu}+\left(\partial_{t} \beta\right) J_{\nu} u_{\nu}\right\|_{W^{k, p}(U)} \\
& \left.+\left\|\left(J_{0}-J_{\nu}\right) \partial_{t} v_{\nu}\right\|_{W^{k, p}(U)}+\left\|v_{\nu}\right\|_{W^{k, p}(U)}\right) \\
\leq & c^{\prime}\left(\left\|\partial_{s} u_{\nu}+J_{\nu} \partial_{t} u_{\nu}\right\|_{W^{k, p}(U)}+\left\|u_{\nu}\right\|_{W^{k, p}(U)}\right. \\
& +\left\|J_{0}-J_{\nu}\right\|_{W^{k, p}(U)}\left\|\partial_{t} v_{\nu}\right\|_{L^{\infty}(U)} \\
& \left.+\left\|J_{0}-J_{\nu}\right\|_{L^{\infty}(U)}\left\|v_{\nu}\right\|_{W^{k+1, p}(U)}\right)
\end{aligned}
$$

for every $\nu$. If $\nu$ is sufficiently large then $c^{\prime}\left\|J_{0}-J_{\nu}\right\|_{L^{\infty}(U)} \leq 1 / 2$ and hence

$$
\begin{aligned}
\left\|v_{\nu}\right\|_{W^{k+1, p}(U)} \leq & 2 c^{\prime}\left(\left\|\partial_{s} u_{\nu}+J_{\nu} \partial_{t} u_{\nu}\right\|_{W^{k, p}(U)}+\left\|u_{\nu}\right\|_{W^{k, p}(U)}\right) \\
& +4 c^{\prime} c_{0}\left\|\partial_{t} v_{\nu}\right\|_{L^{\infty}(U)} .
\end{aligned}
$$


This contradicts the fact that $\left\|u_{\nu}\right\|_{W^{k+1, p}(K)}$ diverges to $\infty$.

Proof of Theorem 3.2. The proof consists of three steps.

Step 1. Fix a constant $p>2$ and a smooth reference connection $A_{0} \in \mathcal{A}(P)$. We may assume without loss of generality that the sequence $A_{\nu}-A_{0}$ is bounded in $W^{1, p}$.

Since $F_{A_{\nu}}=\left(\tau_{\nu}-\mu_{\nu}\left(u_{\nu}\right)\right) \mathrm{dvol}_{\Sigma, \nu}$ it follows from the assumptions that

$$
\sup _{\nu}\left\|F_{A_{\nu}}\right\|_{L^{\infty}}<\infty
$$

Hence, by Uhlenbeck compactness [32, 37], there exists a sequence of gauge transformations $g_{\nu} \in \mathcal{G}^{2, p}(P)$ such that the connections $g_{\nu}^{*} A_{\nu} \in \mathcal{A}^{1, p}(P)$ satisfy a uniform $W^{1, p}$-bound. Replace the sequence $\left(u_{\nu}, A_{\nu}\right)$ by $\left(g_{\nu}^{-1} u_{\nu}, g_{\nu}^{*} A_{\nu}\right)$.

Step 2. We may assume without loss of generality that $A_{\nu}$ converges weakly in $W^{1, p}$ and strongly in $C^{0}$ to a connection $A \in \mathcal{A}^{\ell+1, p}(P)$, that $u_{\nu}$ converges weakly in $W^{1, p}$ and strongly in $C^{0}$ to a section $u \in W_{\mathrm{G}}^{\ell+1, p}(P, M)$, and that

$$
d_{A}^{*}\left(A_{\nu}-A\right)=0
$$

By Step 1, the sequence $A_{\nu}-A_{0}$ is bounded in $W^{1, p}$ and, by assumption, the sequence $u_{\nu}$ is bounded in $W^{1, p}$. Hence, by the theorems of Alaoglu and Rellich, we may assume, after passing to a subsequence if necessary, that $A_{\nu}$ converges weakly in $W^{1, p}$ and strongly in $C^{0}$ to a connection $A_{\infty} \in \mathcal{A}^{1, p}(P)$, and $u_{\nu}$ converges weakly in $W^{1, p}$ and strongly in $C^{0}$ to a section $u_{\infty} \in$ $W^{1, p}(\Sigma, E)$. Since $\bar{\partial}_{J_{\nu}, H_{\nu}, A_{\nu}}\left(u_{\nu}\right)$ converges weakly in $L^{p}$ to $\bar{\partial}_{J, H, A_{\infty}}\left(u_{\infty}\right)$ and $F_{A_{\nu}}$ converges weakly in $L^{p}$ to $F_{A_{\infty}}$ it follows that the limit $\left(u_{\infty}, A_{\infty}\right)$ satisfies (3). By Theorem 3.1, there exists a gauge transformation $g \in$ $\mathcal{G}^{2, p}(P)$ such that the pair

$$
(u, A):=\left(g^{-1} u_{\infty}, g^{*} A_{\infty}\right)
$$

is of class $W^{\ell+1, p}$. Moreover, $g^{*} A_{\nu}$ converges weakly in $W^{1, p}$ and strongly in $C^{0}$ to $A$ and $g^{-1} u_{\nu}$ converges weakly in $W^{1, p}$ and strongly in $C^{0}$ to $u$. By the local slice theorem (Theorem B.1), there exists a sequence of gauge transformations $h_{\nu}$ such that

$$
d_{A}^{*}\left(h_{\nu}^{*} g^{*} A_{\nu}-A\right)=0
$$


and

$$
\lim _{\nu \rightarrow \infty}\left\|h_{\nu}^{*} g^{*} A_{\nu}-A\right\|_{L^{p}}=0, \quad \sup _{\nu}\left\|h_{\nu}^{*} g^{*} A_{\nu}-A\right\|_{W^{1, p}}<\infty .
$$

Passing to a subsequence, if necessary, we may assume that $h_{\nu}^{*} g^{*} A_{\nu}$ converges weakly in the $W^{1, p}$-norm and strongly in the $C^{0}$-norm. The limit is necessarily equal to $A$. Moreover, the sequence $h_{\nu}$ is uniformly bounded in the $W^{2, p}$-norm. Passing to a further subsequence we may assume that $h_{\nu}$ converges weakly in $W^{2, p}$ and strongly in $W^{1, p}$ to a gauge tranformation $h \in \mathcal{G}^{2, p}(P)$. This gauge transformation satisfies

$$
h^{*} A=A
$$

and hence is of class $W^{\ell+2, p}$. It follows that $h_{\nu}^{-1} g^{-1} u_{\nu}$ converges weakly in $W^{1, p}$ and strongly in $C^{0}$ to $h^{-1} u$. Now replace $A_{\nu}$ by $h_{\nu}^{*} g^{*} A_{\nu}, u_{\nu}$ by $h_{\nu}^{-1} g^{-1} u$, and $u$ by $h^{-1} u$.

Step 3. The sequence $\left(u_{\nu}, A_{\nu}-A\right)$ is bounded in $W^{\ell+1, p}$.

We prove, by induction, that $\left(u_{\nu}, A_{\nu}-A\right)$ is bounded in $W^{k, p}$ for $1 \leq$ $k \leq \ell+1$. For $k=1$ this was proved in Step 1. Let $\alpha_{\nu}:=A_{\nu}-A$ and assume, by induction, that the sequence $\left(u_{\nu}, \alpha_{\nu}\right)$ is bounded in $W^{k, p}$ for some $k \in\{1, \ldots, \ell\}$. In local $J_{\Sigma, \nu}$-holomorphic coordinates on $\Sigma$ and local coordinates on $M$ the equation $\bar{\partial}_{J_{\nu}, H_{\nu}, A_{\nu}}\left(u_{\nu}\right)=0$ has the form

$$
\partial_{s} u_{\nu}+J_{\nu}\left(s, t, u_{\nu}\right) \partial_{t} u_{\nu}=-X_{F_{\nu}+\left\langle\mu_{\nu}, \Phi_{\nu}\right\rangle}\left(u_{\nu}\right)-J_{\nu}\left(s, t, u_{\nu}\right) X_{G_{\nu}+\left\langle\mu_{\nu}, \Psi_{\nu}\right\rangle}\left(u_{\nu}\right)
$$

where $A_{\nu}=\Phi_{\nu} d s+\Psi_{\nu} d t$ and $H_{\nu}=F_{\nu} d s+G_{\nu} d t$. This local equation holds in an open set $U \subset \mathbb{C}$, the function $u_{\nu}: U \rightarrow \mathbb{R}^{2 n}$ takes values in an open set $V \subset \mathbb{R}^{2 n}$, the function $J_{\nu}: U \times V \rightarrow \mathcal{J}_{n}$ is of class $C^{\ell}$ (with a uniform $C^{\ell}$-bound), and the functions $F_{\nu}, G_{\nu}: U \times V \rightarrow \mathbb{R}$ are of class $C^{\ell+1}$ (with uniform $C^{\ell+1}$-bounds). Since $u_{\nu}$ is uniformly bounded in $W^{k, p}$ so is the complex structure $U \rightarrow \mathcal{J}_{n}: s+i t \mapsto J_{\nu}\left(s, t, u_{\nu}(s, t)\right)$. Moreover, the sequences $\Phi_{\nu}, \Psi_{\nu}: U \rightarrow \mathfrak{g}$ are bounded in $W^{k, p}$. Hence the sequence $\partial_{s} u_{\nu}+J_{\nu}\left(s, t, u_{\nu}\right) \partial_{t} u_{\nu}: U \rightarrow \mathbb{R}^{2 n}$ is bounded in $W^{k, p}$. By assumption, the sequence $u_{\nu}: U \rightarrow V$ is bounded in $W^{1, \infty}$. Hence, by Lemma 3.3, the sequence $u_{\nu}$ is bounded in $W^{k+1, p}$. Now, by Step 2, we have

$$
d_{A} \alpha_{\nu}=-F_{A}-\frac{1}{2}\left[\alpha_{\nu} \wedge \alpha_{\nu}\right]+\left(\tau_{\nu}-\mu_{\nu}\left(u_{\nu}\right)\right) \operatorname{dvol}_{\Sigma, \nu}, \quad d_{A}^{*} \alpha_{\nu}=0 .
$$

Since the sequences $\alpha_{\nu}$ and $u_{\nu}$ are bounded in $W^{k, p}$ it follows that $d_{A} \alpha_{\nu}$ is bounded in $W^{k, p}$ and hence the sequence $\alpha_{\nu}$ is bounded in $W^{k+1, p}$. This completes the induction. Hence, by Rellich's theorem, the sequence $\left(u_{\nu}, A_{\nu}\right)$ has a $C^{\ell}$-convergent subsequence. 


\subsection{Bubbling.}

The following theorem removes the hypothesis of a uniform $L^{\infty}$ bound on the first derivatives in Theorem 3.2. The manifold $(M, \omega)$ is called symplectically aspherical if

$$
\int_{S^{2}} v^{*} \omega=0
$$

for every smooth map $v: S^{2} \rightarrow M$. This implies that there is no nonconstant $J$-holomorphic sphere (for any almost complex structure on $M$ that is tamed by $\omega)$.

Theorem 3.4. Suppose that $M$ is symplectically aspherical and fix a compact subset $K \subset M$. Let $\ell$ be either a positive integer or be equal to $\infty$. Let $\omega_{\nu}, \mu_{\nu}, \mathrm{dvol}_{\Sigma, \nu}, J_{\Sigma, \nu}, J_{\nu}, H_{\nu}$, and $\tau_{\nu}$ be as in Theorem 3.2. For every $\nu$ let $\left(u_{\nu}, A_{\nu}\right) \in W_{\mathrm{G}}^{1, p}(P, M) \times \mathcal{A}^{1, p}(P)$ be a solution of (3) with $\left(\mu, J_{\Sigma}, \operatorname{dvol}_{\Sigma}, J, H, \tau\right)$ replaced by $\left(\mu_{\nu}, J_{\Sigma, \nu}, \operatorname{dvol}_{\Sigma, \nu}, J_{\nu}, H_{\nu}, \tau_{\nu}\right)$ such that

$$
u_{\nu}(P) \subset K
$$

for every $\nu$ and

$$
\sup _{\nu}\left\langle\left[\omega_{\nu}-\mu_{\nu}\right],\left[u_{\nu}\right]\right\rangle<\infty .
$$

Then there exists sequence of gauge transformations $g_{\nu} \in \mathcal{G}^{2, p}(P)$ such that the sequence $\left(g_{\nu}^{-1} u_{\nu}, g_{\nu}^{*} A_{\nu}\right)$ has a $C^{\ell}$-convergent subsequence.

Proof. By Theorem 3.2, it suffices to prove that

$$
\sup _{\nu}\left\|d_{A_{\nu}} u_{\nu}\right\|_{L^{\infty}}<\infty
$$

Fix a constant $p>2$. By Step 1 in the proof of Theorem 3.2, we may assume that the sequence $A_{\nu}-A_{0}$ satisfies a uniform $W^{1, p}$-bound for some (and hence every) smooth connection $A_{0}$. Now suppose, by contradiction, that the sequence $\left\|d_{A_{\nu}} u_{\nu}\right\|_{L^{\infty}}$ is unbounded. Passing to a subsequence, if necessary, we may assume that this sequence diverges to $\infty$. Choose a sequence $p_{\nu} \in P$ such that

$$
c_{\nu}:=\left|d_{A_{\nu}} u_{\nu}\left(p_{\nu}\right)\right|=\left\|d_{A_{\nu}} u_{\nu}\right\|_{L^{\infty}} \rightarrow \infty .
$$

Passing to a subsequence, we may assume without loss of generality that $p_{\nu}$ converges. Let $p_{\infty}:=\lim _{\nu \rightarrow \infty} p_{\nu}$ and $z_{\infty}:=\pi\left(p_{\infty}\right)$. Choose a convergent 
sequence of local $J_{\Sigma, \nu}$-holomorphic coordinates $s+i t$ on $\Sigma$ near $z_{\infty}$ and lift these to a convergent sequence of local sections of $P$ that pass at the origin through $p_{\infty}$. In this local frame equations (3) have the form

$$
\begin{aligned}
\partial_{s} u_{\nu}+X_{F_{\nu}+\left\langle\mu_{\nu}, \Phi_{\nu}\right\rangle}\left(u_{\nu}\right)+J_{\nu}\left(s, t, u_{\nu}\right)\left(\partial_{t} u_{\nu}+X_{G_{\nu}+\left\langle\mu_{\nu}, \Psi_{\nu}\right\rangle}\left(u_{\nu}\right)\right) & =0 \\
\partial_{s} \Psi_{\nu}-\partial_{t} \Phi_{\nu}+\left[\Phi_{\nu}, \Psi_{\nu}\right]+\lambda^{2}\left(\mu\left(u_{\nu}\right)-\tau_{\nu}\right) & =0 .
\end{aligned}
$$

Now consider the rescaled sequence

$$
v_{\nu}(s, t):=u_{\nu}\left(\varepsilon_{\nu} s, \varepsilon_{\nu} t\right), \quad \varepsilon_{\nu}:=\frac{1}{c_{\nu}} .
$$

This sequence satisfies

$$
\partial_{s} v_{\nu}+J_{\nu}\left(\varepsilon_{\nu} s, \varepsilon_{\nu} t, v_{\nu}\right) \partial_{t} v_{\nu}=-\varepsilon_{\nu} w_{\nu}
$$

where

$$
\begin{aligned}
w_{\nu}:= & X_{\Phi_{\nu}\left(\varepsilon_{\nu} s, \varepsilon_{\nu} t\right)}\left(v_{\nu}\right)+X_{F_{\nu}}\left(\varepsilon_{\nu} s, \varepsilon_{\nu} t, v_{\nu}\right) \\
& +J_{\nu}\left(\varepsilon_{\nu} s, \varepsilon_{\nu} t, v_{\nu}\right)\left(X_{\Psi_{\nu}\left(\varepsilon_{\nu} s, \varepsilon_{\nu} t\right)}\left(v_{\nu}\right)+X_{G_{\nu}}\left(\varepsilon_{\nu} s, \varepsilon_{\nu} t, v_{\nu}\right)\right)
\end{aligned}
$$

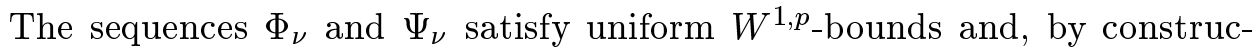
tion, the sequence $v_{\nu}$ satisfies a uniform $W^{1, \infty}$-bound on every compact set. Hence the sequence $w_{\nu}$ satisfies a uniform $W^{1, p}$-bound on every compact set. By Lemma 3.3, the sequence $v_{\nu}$ satisfies a uniform $W^{2, p_{\text {-bound on }}}$ every compact set. It follows that $v_{\nu}$ has a subsequence which converges strongly in $C^{1}$ on every compact set. The limit is a nonconstant pseudoholomorphic curve $v: \mathbb{C} \rightarrow K$ with respect to the almost complex structure $J_{\infty}:=J\left(z_{\infty}, \cdot\right)$. We prove that it has finite energy. To see this note that, for every $R>0$,

$$
\begin{aligned}
& E\left(v ; B_{R}\right) \\
& =\int_{B_{R}}\left|\partial_{s} v\right|_{J_{\infty}}^{2} \\
& =\lim _{\nu \rightarrow \infty} \int_{B_{R}}\left|\partial_{s} v^{\nu}+\varepsilon_{\nu} X_{\Phi_{\nu}\left(\varepsilon_{\nu} s, \varepsilon_{\nu} t\right)}\left(v_{\nu}\right)+\varepsilon_{\nu} X_{F_{\nu}}\left(\varepsilon_{\nu} s, \varepsilon_{\nu} t, v_{\nu}\right)\right|_{J_{\nu}\left(\varepsilon_{\nu} s, \varepsilon_{\nu} t, v_{\nu}\right)}^{2} \\
& =\lim _{\nu \rightarrow \infty} \int_{B_{\varepsilon_{\nu} R}}\left|\partial_{s} u_{\nu}+X_{\Phi_{\nu}}\left(u_{\nu}\right)+X_{F_{\nu}}\left(s, t, u_{\nu}\right)\right|_{J_{\nu}\left(s, t, u_{\nu}\right)}^{2} \\
& \leq \limsup _{\nu \rightarrow \infty} E_{\nu}\left(u_{\nu}, A_{\nu}\right) .
\end{aligned}
$$


By (16) and the energy identity in Proposition 2.2, the sequence $E\left(u_{\nu}, A_{\nu}\right)$ is bounded. Hence $v$ has finite energy, and hence, by the removable singularity theorem (see for example [23, Theorem 4.2.1]), it extends to a nonconstant $J_{\infty}$-holomorphic sphere in $K$. Since $M$ is symplectically aspherical such a $J_{\infty}$-holomorphic sphere does not exist. This contradiction proves (17).

Combining Theorem 3.4 with the apriori estimate of Lemma 2.7 we obtain the following compactness result for the moduli space of solutions of (3).

Corollary 3.5. Suppose $(M, \omega, \mu)$ is symplectically aspherical and admits a strongly convex structure $\left(f, J_{0}\right)$ as in Section 2.5 . Let $\ell$ be either a positive integer or be equal to $\infty$. Let $\omega_{\nu}, \mu_{\nu}, \mathrm{dvol}_{\Sigma, \nu}, J_{\Sigma, \nu}, J_{\nu}, H_{\nu}$, and $\tau_{\nu}$ be as in Theorem 3.2. Let $c_{0}: Z(\mathfrak{g}) \rightarrow(0, \infty)$ be as in $\left(C 3^{\prime}\right)$, suppose that $(11)$ holds with $c_{0}=c_{0}(\tau)$, that each $J_{\nu}$ agrees with $J_{0}$ outside of the compact set

$$
M_{0}:=\left\{x \in M \mid f(x) \leq c_{0}(\tau)+1\right\},
$$

and that each Hamiltonian $H_{\nu}$ is supported in $M_{0}$. Then, for every sequence $\left(u_{\nu}, A_{\nu}\right)$ of $W^{1, p}$-solutions of $(3)$, with the tuple $\left(\mu, J_{\Sigma}, \mathrm{dvol}_{\Sigma}, J, H, \tau\right)$ replaced by $\left(\mu_{\nu}, J_{\Sigma, \nu}, \operatorname{dvol}_{\Sigma, \nu}, J_{\nu}, H_{\nu}, \tau_{\nu}\right)$, such that $u_{\nu}$ represents a fixed equivariant homology class there exists a sequence of gauge transformations $g_{\nu} \in \mathcal{G}^{2, p}(P)$ such that $\left(g_{\nu}^{-1} u_{\nu}, g_{\nu}^{*} A_{\nu}\right)$ has a $C^{\ell}$-convergent subsequence.

Proof. By Lemma 2.7, $u_{\nu}(P) \subset M_{0}$ for every $\nu$. Hence the result follows from Theorem 3.4 with $K=M_{0}$.

\section{Fredholm theory.}

Fix a symplectic $2 n$-manifold $(M, \omega)$ with a Hamiltonian G-action and moment map $\mu: M \rightarrow \mathfrak{g}$, a compact Riemann surface $\left(\Sigma, J_{\Sigma}, \mathrm{dvol}_{\Sigma}\right)$, an almost complex structure $J \in \mathcal{J}$, a Hamiltonian perturbation $H \in \mathcal{H}$, an equivariant homology class $B \in H_{2}\left(M_{\mathrm{G}} ; \mathbb{Z}\right)$, and a principal G-bundle $P \rightarrow \Sigma$ whose characteristic class $[P] \in H_{2}(\mathrm{BG} ; \mathbb{Z})$ is the image of $B$ under the homomorphism $H_{2}\left(M_{\mathrm{G}} ; \mathbb{Z}\right) \rightarrow H_{2}(\mathrm{BG} ; \mathbb{Z})$. In this section we examine the moduli space

$$
\begin{aligned}
\widetilde{\mathcal{M}}_{B, \Sigma} & :=\widetilde{\mathcal{M}}_{B, \Sigma}(\tau ; J, H) \\
& :=\left\{(u, A) \in W_{\mathrm{G}}^{1, p}(P, M) \times \mathcal{A}^{1, p}(P) \mid(u, A) \text { satisfy }(3),[u]=B\right\} .
\end{aligned}
$$

The quotient by the action of the gauge group will be denoted by

$$
\mathcal{M}_{B, \Sigma}:=\mathcal{M}_{B, \Sigma}(\tau ; J, H):=\widetilde{\mathcal{M}}_{B, \Sigma}(\tau ; J, H) / \mathcal{G}^{2, p}(P) .
$$


In this section we prove that, for a generic Hamiltonian perturbation, the subspace $\mathcal{M}_{B, \Sigma}^{*}$ of irreducible solutions is a finite dimensional manifold.

\subsection{Regular and irreducible solutions.}

Let $J \in \mathcal{J}^{\ell}$ and $H \in \mathcal{H}^{\ell}$ (see Sections 2.2 and 3.1 for the notation). We denote by $\mathrm{G}_{x}:=\{g \in \mathrm{G} \mid g x=x\}$ the isotopy subgroup of $x \in M$.

Definition 4.1. A solution $(u, A) \in \mathrm{W}_{\mathrm{G}}^{1, p}(P, M) \times \mathcal{A}^{1, p}(P)$ of $(3)$ is called regular if

$$
d_{A} \eta=0, \quad L_{u} \eta=0 \quad \Longrightarrow \quad \eta=0
$$

for every $\eta \in W^{2, p}\left(\Sigma, \mathfrak{g}_{P}\right)$. It is called irreducible if there exists a point $p \in P$ such that

$$
\mathrm{G}_{u(p)}=\{\mathbb{1}\}, \quad \operatorname{im} L_{u(p)} \cap \operatorname{im} J L_{u(p)}=\{0\} .
$$

Note that every irreducible solution is regular. Note also that an element $\tau \in Z(\mathfrak{g})$ is a regular value of the restriction of the moment map

$$
\mathrm{W}_{\mathrm{G}}^{1, p}(P, M) \times \mathcal{A}^{1, p}(P) \rightarrow L^{p}\left(\Sigma, \mathfrak{g}_{P}\right):(u, A) \mapsto * F_{A}+\mu(u)
$$

to the space of pairs $(u, A)$ such that $\bar{\partial}_{J, H, A}(u)=0$ and $[u]=B$ if and only if every pair $(u, A) \in \widetilde{\mathcal{M}}_{B, \Sigma}(\tau ; J, H)$ is regular. The next lemma shows that, if $\tau$ is a regular value of $\mu$ then regularity can be achieved by choosing a Riemann surface with large volume.

Lemma 4.2. Let $\tau \in Z(\mathfrak{g})$.

(i) If $\tau$ is a regular value of $\mu$ then there exists a constant $\delta>0$ with the following significance. If $B, \Sigma$, and $H$ satisfy

$$
\frac{\langle[\omega+\tau-\mu], B\rangle+\left\|\Omega_{H}\right\|}{\operatorname{Vol}(\Sigma)} \leq \delta
$$

then every solution $(u, A)$ of (3) (for every $J \in \mathcal{J}^{\ell}$ ) with $[u]=B$ is regular.

(ii) If $\mathrm{G}$ acts freely on $\mu^{-1}(\tau)$ then there exists a constant $\delta>0$ with the following significance. If $B, \Sigma$, and $H$ satisfy (19) then every solution $(u, A)$ of $(3)$ (for every $\left.J \in \mathcal{J}^{\ell}\right)$ with $[u]=B$ is irreducible. 
Proof. Choose $\delta>0$ such that

$$
|\mu(x)-\tau|^{2} \leq \delta \quad \Longrightarrow \quad \text { ker } L_{x}=\{0\} .
$$

Let $(u, A) \in \mathrm{W}_{\mathrm{G}}^{1, p}(P, M) \times \mathcal{A}^{1, p}(P)$, be a solution of $(3)$ such that $[u]=B$. Then, by Proposition 2.2, we have

$$
\begin{aligned}
\inf _{p \in P}|\mu(u(p))-\tau|^{2} & \leq \frac{1}{\operatorname{Vol}(\Sigma)} \int_{\Sigma}|\mu(u)-\tau|^{2} \mathrm{dvol}_{\Sigma} \\
& \leq \frac{E(u, A)}{\operatorname{Vol}(\Sigma)} \\
& \leq \frac{\langle[\omega+\tau-\mu], B\rangle+\left\|\Omega_{H}\right\|}{\operatorname{Vol}(\Sigma)} \\
& \leq \delta .
\end{aligned}
$$

The last inequality follows from (19). Hence there exists a point $p_{0} \in P$ such that $\left|\mu\left(u\left(p_{0}\right)\right)-\tau\right|^{2} \leq \delta$ and so, by definiton of $\delta$, the linear map $L_{u\left(p_{0}\right)}: \mathfrak{g} \rightarrow T_{u\left(p_{0}\right)} M$ is injective. Now suppose that $\eta \in W^{1, p}\left(\Sigma, \mathfrak{g}_{P}\right)$ satisfies

$$
d_{A} \eta=0, \quad L_{u} \eta=0 .
$$

Then $\eta\left(p_{0}\right)=0$ and hence $\eta \equiv 0$. This proves (i). To prove (ii) choose $\delta>0$ such that

$$
|\mu(x)-\tau|^{2} \leq \delta \quad \Longrightarrow \quad \mathrm{G}_{x}=\{\mathbb{1}\}, \operatorname{im} L_{x} \cap \operatorname{im} J(z, x) L_{x}=\{0\}
$$

for all $(z, x) \in \Sigma \times M$ and argue as in the proof of (i).

Given $\tau \in Z(\mathfrak{g})$ and $(J, H) \in \mathcal{J} \times \mathcal{H}$, we denote the set of irreducible solutions of (3) by

$\widetilde{\mathcal{M}}_{B, \Sigma}^{*}:=\widetilde{\mathcal{M}}_{B, \Sigma}^{*}(M, \omega, \mu, \tau ; J, H):=\left\{(u, A) \in \widetilde{\mathcal{M}}_{B, \Sigma} \mid(u, A)\right.$ is irreducible $\}$

and the quotient space by

$$
\mathcal{M}_{B, \Sigma}^{*}:=\mathcal{M}_{B, \Sigma}^{*}(M, \omega, \mu, \tau ; J, H):=\widetilde{\mathcal{M}}_{B, \Sigma}^{*}(M, \omega, \mu, \tau ; J, H) / \mathcal{G}^{2, p}(P) .
$$

Remark 4.3. The regularity criterion of Lemma 4.2 is useful in certain situations (e.g. for the adiabatic limit argument in [15]). However, the condition is rather restrictive and in many cases the solutions are regular 
under much more general hypotheses. For example, in the case of linear torus actions, one can consider the element $\tau_{0} \in \mathfrak{g}$ defined by

$$
\tau_{0}:=\tau-\frac{1}{\operatorname{Vol}(\Sigma)} \int_{\Sigma} F_{A}
$$

for $A \in \mathcal{A}(P)$. This element is independent of the connection $A$. Suppose $M=\mathbb{C}^{n}$ and $\mathrm{G}$ is abelian and acts linearly on $M$. If $\mathrm{G}$ and acts freely (respectively with finite isotropy) on $\mu^{-1}\left(\tau_{0}\right)$ then the gauge group acts freely (respectively with finite isotropy) on the space of solutions of (3) for every Hamiltonian perturbation. To see this note that, for every subgroup $\mathrm{H} \subset \mathrm{G}$, the set

$$
M^{\mathrm{H}}:=\left\{x \in M \mid \mathrm{H} \subset \mathrm{G}_{x}\right\}
$$

is a linear subspace of $M=\mathbb{C}^{n}$ and so $\mu\left(M^{H}\right)$ is a closed convex cone. Applying this to the subgroup

$$
\mathrm{H}:=\bigcap_{p \in P} \mathrm{G}_{u(p)},
$$

where $* F_{A}+\mu(u)=\tau$, we find that

$$
\tau_{0}=\frac{1}{\operatorname{Vol}(\Sigma)} \int_{\Sigma} \mu(u) \operatorname{dvol}_{\Sigma} \in \mu\left(M^{H}\right) .
$$

Hence, if $\mathrm{G}$ acts freely on $\mu^{-1}\left(\tau_{0}\right)$, it follows that $\mathrm{H}=\{\mathbb{1}\}$ and, if $\tau_{0}$ is a regular value of $\mu$, it follows that $\mathrm{H}$ is finite. Since $\mathrm{H}$ is isomorphic to the isotropy subgroup of the pair $(u, A)$, this proves the claim.

\subsection{The linearized operator.}

Cauchy-Riemann operators. Fix an almost complex structure $J \in \mathcal{J}$ and a Hamiltonian perturbation $H \in \mathcal{H}$. We begin with a discussion of the Cauchy-Riemann operator on the vector bundle $E_{u}:=u^{*} T M / \mathrm{G} \rightarrow \Sigma$ associated to a pair $(u, A) \in C_{\mathrm{G}}^{\infty}(P, M) \times \mathcal{A}(P)$. This operator will be denoted by $D_{u, A}: C^{\infty}\left(\Sigma, E_{u}\right) \rightarrow \Omega^{0,1}\left(\Sigma, E_{u}\right)$. It is obtained by differentiating the first equation in (3) with respect to $u$ and is given by

$$
D_{u, A} \xi=\left(\nabla_{H, A} \xi\right)^{0,1}-\frac{1}{2} J\left(\nabla_{\xi} J\right) \partial_{J, H, A}(u)
$$

for $\xi \in C^{\infty}\left(\Sigma, E_{u}\right)$. Here $\nabla$ denotes the Levi-Civita connection of the metric $\omega(\cdot, J \cdot)$ on $M$. Since $J$ depends on the basepoint $z \in \Sigma$ so does the connection $\nabla$. The connection $\nabla_{H, A}$ on $E_{u}$ is given by

$$
\nabla_{H, A} \xi=\nabla \xi+\nabla_{\xi} X_{H, A}(u)
$$


where the 1 -form $X_{H, A}: T P \rightarrow \operatorname{Vect}(M, \omega)$ is given by

$$
\left(X_{H, A}\right)_{p}(v):=X_{A_{p}(v)}+X_{H_{d \pi(p) v}}
$$

for $v \in T_{p} P$.

Remark 4.4. In conformal coordinates $s+i t$ on $\Sigma$ the connection $\nabla_{H, A}$ has the form

$$
\nabla_{H, A, s} \xi=\nabla_{s} \xi+\nabla_{\xi} X_{\Phi}+\nabla_{\xi} X_{F}, \quad \nabla_{H, A, t} \xi=\nabla_{t} \xi+\nabla_{\xi} X_{\Psi}+\nabla_{\xi} X_{G}
$$

Here $\Omega \subset \mathbb{C}$ is an open set, $u: \Omega \rightarrow M$ is a smooth function, $\nabla$ denotes the Levi-Civita connection of the metric $\langle\cdot, \cdot\rangle_{s, t}=\omega\left(\cdot, J_{s, t^{*}}\right)$, and

$$
A=\Phi d s+\Psi d t, \quad H=F d s+G d t
$$

where $\Phi, \Psi: \Omega \rightarrow \mathfrak{g}$ and $F, G: \Omega \times M \rightarrow \mathbb{R}$. Thus the Cauchy-Riemann operator has the form

$$
D_{u, A} \xi=\frac{1}{2}\left(\xi^{\prime} d s+J \xi^{\prime} d t\right)
$$

where

$$
\xi^{\prime}=\nabla_{H, A, s} \xi+J \nabla_{H, A, t} \xi-\frac{1}{2} J\left(\nabla_{\xi} J\right)\left(v_{s}-J v_{t}\right)
$$

and

$$
v_{s}:=\partial_{s} u+L_{u} \Phi+X_{F}(u), \quad v_{t}:=\partial_{t} u+L_{u} \Psi+X_{G}(u) .
$$

The covariant derivatives of $J=J(s, t, u(s, t))$ with respect to the connection $\nabla_{H, A}$ are given by

$$
\nabla_{H, A, s} J=\nabla_{v_{s}} J+\partial_{s} J-\mathcal{L}_{X_{F}} J, \quad \nabla_{H, A, t} J=\nabla_{v_{t}} J+\partial_{t} J-\mathcal{L}_{X_{G}} J .
$$

Remark 4.5. We obtain a Hermitian connection $\widetilde{\nabla}_{H, A}$ on $u^{*} T M$ by the formula

$$
\widetilde{\nabla}_{H, A} \xi:=\nabla_{H, A} \xi-\frac{1}{2} J\left(\nabla_{H, A} J\right) \xi .
$$

The complex linear part of $D_{u, A}$ is given by $\xi \mapsto\left(\widetilde{\nabla}_{H, A} \xi\right)^{0,1}$ and, moreover,

$$
D_{u, A} \xi=\left(\widetilde{\nabla}_{H, A} \xi\right)^{0,1}+\frac{1}{4} N\left(\xi, \partial_{J, H, A}(u)\right)+\frac{1}{2}\left(J\left(\mathcal{L}_{X_{H}} J-\dot{J}\right) \xi\right)^{0,1}
$$

(see [15]). Here

$$
N\left(\xi_{1}, \xi_{2}\right)=2 J\left(\nabla_{\xi_{2}} J\right) \xi_{1}-J\left(\nabla_{\xi_{1}} J\right) \xi_{2}
$$

denotes the Nijenhuis tensor of $J=J_{z}$. 
An abstract setting. Consider the infinite dimensional Banach manifold

$$
\mathcal{B}:=\mathcal{B}^{k, p}:=W_{\mathrm{G}}^{k, p}(P, M) \times \mathcal{A}^{k, p}(P) .
$$

The tangent space of $\mathcal{B}$ at $(u, A) \in \mathcal{B}$ is given by

$$
T_{(u, A)} \mathcal{B}=W^{k, p}\left(\Sigma, E_{u}\right) \times W^{k, p}\left(\Sigma, T^{*} \Sigma \otimes \mathfrak{g}_{P}\right), \quad E_{u}:=u^{*} T M / \mathrm{G} .
$$

The almost complex structure $J \in \mathcal{J}$ determines a complex structure $J_{u}$ on $E_{u}$ and hence a vector bundle $\mathcal{E}=\mathcal{E}^{k-1, p} \rightarrow \mathcal{B}$ with fibres

$$
\mathcal{E}_{(u, A)}:=\mathcal{E}_{u}:=W^{k-1, p}\left(\Sigma, \Lambda_{J_{u}}^{0,1} T^{*} \Sigma \otimes E_{u}\right) \times W^{k-1, p}\left(\Sigma, \mathfrak{g}_{P}\right) .
$$

The action of the gauge group $\mathcal{G}^{2, p}(P)$ on $\mathcal{B}$ lifts to an action on $\mathcal{E}$. For every Hamiltonian perturbation $H \in \mathcal{H}$ there is a $\mathcal{G}^{2, p}(P)$-equivariant section

$$
\mathcal{F}=\mathcal{F}_{H, J}: \mathcal{B} \rightarrow \mathcal{E}
$$

given by

$$
\mathcal{F}_{H, J}(u, A):=\left(\bar{\partial}_{J, H, A}(u), * F_{A}+\mu(u)-\tau\right) .
$$

The space $\widetilde{\mathcal{M}}_{B, \Sigma}(M, \omega, \mu, \tau ; J, H)$ is the zero set of this section.

The linearized operator. The vertical differential of $\mathcal{F}$ at a zero $(u, A)$ gives rise to an operator

$$
\mathcal{D}_{u, A}: T_{(u, A)} \mathcal{B}^{k, p} \rightarrow \mathcal{E}_{u}^{k-1, p} \oplus W^{k-1, p}\left(\Sigma, \mathfrak{g}_{P}\right)
$$

given by

$$
\mathcal{D}_{u, A}\left(\begin{array}{c}
\xi \\
\alpha
\end{array}\right)=\left(\begin{array}{c}
D_{u, A} \xi+\left(L_{u} \alpha\right)^{0,1} \\
L_{u}^{*} \xi-d_{A}^{*} \alpha \\
d \mu(u) \xi+* d_{A} \alpha
\end{array}\right)
$$

Here the linear map $L_{x}: \mathfrak{g} \rightarrow T_{x} M$ is given by the infinitesmal action, i.e.

$$
L_{x} \eta:=X_{\eta}(x)
$$

for $x \in M$ and $\eta \in \mathfrak{g}$, and $L_{x}^{*}: T_{x} M \rightarrow \mathfrak{g}$ denotes its dual operator with respect to the given invariant inner product on $\mathfrak{g}$ and the inner product $\omega(\cdot, J(z, x) \cdot)$ on $T_{x} M$. Note that this inner product, in general, depends both on $x \in M$ and on the point $z \in \Sigma$. 
Proposition 4.6. Assume $J \in \mathcal{J}^{\ell}$ and $H \in \mathcal{H}^{\ell+1}$ and let $k \in\{1, \ldots, \ell+1\}$ and $p>2$. Then the operator $\mathcal{D}_{u, A}: T_{(u, A)} \mathcal{B}^{k, p} \rightarrow \mathcal{E}_{u}^{k-1, p} \oplus W^{k-1, p}\left(\Sigma, \mathfrak{g}_{P}\right)$, defined by (23), is a Fredholm operator for every pair $(u, A) \in \mathcal{B}^{k, p}$. It has real index

$$
\operatorname{index} \mathcal{D}_{u, A}=(n-\operatorname{dim} \mathrm{G}) \chi(\Sigma)+2\left\langle c_{1}^{\mathrm{G}}(T M),[u]\right\rangle
$$

where $c_{1}^{\mathrm{G}}(T M) \in H^{2}\left(M_{\mathrm{G}} ; \mathbb{Z}\right)$ denotes the first Chern class of the vertical tangent bundle $T M \times_{\mathrm{G}} \mathrm{EG} \rightarrow M \times_{\mathrm{G}} \mathrm{EG}=M_{\mathrm{G}}$.

Proof. The operator

$$
\Omega^{1}\left(\Sigma, \mathfrak{g}_{P}\right) \rightarrow \Omega^{0}\left(\Sigma, \mathfrak{g}_{P}\right) \oplus \Omega^{0}\left(\Sigma, \mathfrak{g}_{P}\right): \alpha \mapsto\left(-d_{A}^{*} \alpha, * d_{A} \alpha\right)
$$

has index $-\chi(\Sigma) \operatorname{dim} \mathrm{G}$ and, by the Riemann-Roch theorem, the CauchyRiemann operator $D_{u, A}: C^{\infty}\left(\Sigma, E_{u}\right) \rightarrow \Omega^{0,1}\left(\Sigma, E_{u}\right)$ has index $n \chi(\Sigma)+$ $2 c_{1}\left(E_{u}\right)$, where $c_{1}\left(E_{u}\right):=\left\langle c_{1}^{\mathrm{G}}(T M),[u]\right\rangle$ denotes the first Chern number of the complex vector bundle $E_{u} \rightarrow \Sigma$. The operator $\mathcal{D}_{u, A}$ is a compact perturbation of the direct sum of these operators.

The adjoint operator. The formal $L^{2}$-adjoint operator

$$
\mathcal{D}_{u, A}^{*}: \mathcal{E}_{u}^{k+1, p} \oplus W^{k+1, p}\left(\Sigma, \mathfrak{g}_{P}\right) \rightarrow T_{(u, A)} \mathcal{B}^{k, p}
$$

is given by

$$
\mathcal{D}_{u, A}^{*}\left(\begin{array}{c}
\eta \\
\phi \\
\psi
\end{array}\right)=\left(\begin{array}{c}
D_{u, A}^{*} \eta+L_{u} \phi+J L_{u} \psi \\
L_{u}^{*} \eta-d_{A} \phi-* d_{A} \psi
\end{array}\right)
$$

for $\eta \in \Omega_{J_{u}}^{0,1}(\Sigma, E)$ and $\phi, \psi \in \Omega^{0}\left(\Sigma, \mathfrak{g}_{P}\right)$.

Proposition 4.7. If $u$ and $A$ satisfy (3) then

$$
\mathcal{D}_{u, A} \mathcal{D}_{u, A}^{*}\left(\begin{array}{c}
\eta \\
\phi \\
\psi
\end{array}\right)=\left(\begin{array}{c}
D_{u, A} D_{u, A}^{*} \eta+\left(L_{u} L_{u}^{*} \eta\right)^{0,1}+\left(D_{u, A} J-J D_{u, A}\right) L_{u} \psi \\
d_{A}^{*} d_{A} \phi+L_{u}^{*} L_{u} \phi \\
d_{A}^{*} d_{A} \psi+L_{u}^{*} L_{u} \psi+L_{u}^{*}\left(D_{u, A} J-J D_{u, A}\right)^{*} \eta
\end{array}\right)
$$

for $\eta \in \Omega_{J_{u}}^{0,1}\left(\Sigma, E_{u}\right)$ and $\phi, \psi \in \Omega^{0}\left(\Sigma, \mathfrak{g}_{P}\right)$. 
Proof. We shall abbreviate $D:=D_{u, A}$ and use the identities

$$
\begin{gathered}
d_{A}^{*} \alpha=-* d_{A} * \alpha, \quad * d_{A} d_{A} \phi=\left[* F_{A}, \phi\right], \\
d \mu(u) J=L_{u}^{*}, \quad d \mu(u) L_{u} \phi=[\phi, \mu(u)]
\end{gathered}
$$

for $\alpha \in \Omega^{1}\left(\Sigma, \mathfrak{g}_{P}\right)$ and $\phi \in \Omega^{0}\left(\Sigma, \mathfrak{g}_{P}\right)$. With this understood we obtain

$$
\mathcal{D}_{u, A} \mathcal{D}_{u, A}^{*}(\eta, \phi, \psi)=(\tilde{\eta}, \tilde{\phi}, \tilde{\psi}),
$$

where

$$
\begin{aligned}
\tilde{\eta}= & D\left(D^{*} \eta+L_{u} \phi+J L_{u} \psi\right)+\left(L_{u}\left(L_{u}^{*} \eta-d_{A} \phi-* d_{A} \psi\right)\right)^{0,1} \\
= & D D^{*} \eta+\left(L_{u} L_{u}^{*} \eta\right)^{0,1} \\
& +D L_{u} \phi-\left(L_{u} d_{A} \phi\right)^{0,1}+D J L_{u} \psi-\left(L_{u} * d_{A} \psi\right)^{0,1}, \\
\tilde{\phi}= & L_{u}^{*}\left(D^{*} \eta+L_{u} \phi+J L_{u} \psi\right)-d_{A}^{*}\left(L_{u}^{*} \eta-d_{A} \phi-* d_{A} \psi\right) \\
= & d_{A}^{*} d_{A} \phi+L_{u}^{*} L_{u} \phi+L_{u}^{*} D^{*} \eta-d_{A}^{*} L_{u}^{*} \eta+\left[* F_{A}+\mu(u), \psi\right], \\
\tilde{\psi}= & d \mu(u)\left(D^{*} \eta+L_{u} \phi+J L_{u} \psi\right)+* d_{A}\left(L_{u}^{*} \eta-d_{A} \phi-* d_{A} \psi\right) \\
= & d_{A}^{*} d_{A} \psi+L_{u}^{*} L_{u} \psi+L_{u}^{*} J^{*} D^{*} \eta+d_{A}^{*} * L_{u}^{*} \eta-\left[* F_{A}+\mu(u), \phi\right] .
\end{aligned}
$$

The assertion then follows from the fact that

$$
J\left(L_{u} \alpha\right)^{0,1}=\left(L_{u} * \alpha\right)^{0,1}, \quad L_{u}^{*} J^{*} \eta=-* L_{u}^{*} \eta,
$$

for $\alpha \in \Omega^{1}\left(\Sigma, \mathfrak{g}_{P}\right)$ and $\eta \in \Omega_{J_{u}}^{0,1}\left(\Sigma, E_{u}\right)$, and

$$
\bar{\partial}_{J, H, A}(u)=0 \quad \Longrightarrow \quad D L_{u} \phi=\left(L_{u} d_{A} \phi\right)^{0,1}
$$

for $\phi \in \Omega^{0}\left(\Sigma, \mathfrak{g}_{P}\right)$. The first equation in (25) follows from the fact that

$$
* \alpha=-\alpha \circ J_{\Sigma}
$$

for every 1-form $\alpha$ on $\Sigma$ (with values in any vector bundle) and hence

$$
\begin{aligned}
\left(L_{u} \alpha\right)^{0,1} & =\frac{1}{2}\left(L_{u} \alpha+J L_{u}\left(\alpha \circ J_{\Sigma}\right)\right) \\
& =\frac{1}{2}\left(\left(L_{u} * \alpha\right) \circ J_{\Sigma}-J L_{u} * \alpha\right) \\
& =-J\left(L_{u} * \alpha\right)^{0,1} .
\end{aligned}
$$

The second equation in (25) follows from the first by duality. Next we observe that the operator $(u, A) \mapsto \bar{\partial}_{J, H, A}(u)$ is a section of the bundle over $\mathcal{B}$ 
with fibres $\Omega_{J_{u}}^{0,1}\left(\Sigma, E_{u}\right)$. Its vertical differential at a zero $(u, A)$ is the operator $(\xi, \alpha) \mapsto D_{u, A} \xi+\left(L_{u} \alpha\right)^{0,1}$. Since the section $(u, A) \mapsto \bar{\partial}_{J, H, A}(u)$ is equivariant under the action of $\mathcal{G}(P)$ it follows that the pair $(\xi, \alpha)=\left(L_{u} \phi,-d_{A} \phi\right)$ is contained in the kernel of the vertical differential at any zero $(u, A)$. This proves $(26)$.

Proposition 4.8. Let $\tau \in Z(\mathfrak{g})$. Then the following holds.

(i) If $(u, A)$ is a regular solution of $(3)$ then $\mathcal{D}_{u, A}^{*}(\eta, \phi, \psi)=0$ implies $\phi=0$.

(ii) If $H=0, \dot{J}=0, J$ is integrable, and $(u, A)$ is a regular solution of (3) then $\mathcal{D}_{u, A}^{*}(\eta, \phi, \psi)=0$ implies $\phi=\psi=0, D_{u, A}^{*} \eta=0$, and $L_{u}^{*} \eta=0$.

(iii) If $H=0, \dot{J}=0, J$ is integrable, $(u, A)$ is a regular solution of $(3)$, and $L_{u(p)}: \mathfrak{g} \rightarrow T_{u(p)} M$ is onto for some $p \in P$ then $\mathcal{D}_{u, A}$ is onto.

(iv) If $H=0, \dot{J}=0, d_{A} u \equiv 0, \mu(u) \equiv \tau, \Sigma=S^{2}$, and $(u, A)$ is irreducible then $\mathcal{D}_{u, A}$ is onto.

Proof. If $u$ and $A$ satisfy (3) and $\mathcal{D}_{u, A}^{*}(\eta, \phi, \psi)=0$ then, by Proposition 4.7, $d_{A} \phi=0$ and $L_{u} \phi=0$. Since $(u, A)$ is regular it follows that $\phi=0$. This proves (i). If $u$ and $A$ satisfy (1) and $J$ is integrable then

$$
D_{u, A} J=J D_{u, A}
$$

and, by Proposition 4.7, we have

$$
\mathcal{D}_{u, A} \mathcal{D}_{u, A}^{*}\left(\begin{array}{c}
\eta \\
\phi \\
\psi
\end{array}\right)=\left(\begin{array}{c}
D_{u, A} D_{u, A}^{*} \eta+\left(L_{u} L_{u}^{*} \eta\right)^{0,1} \\
d_{A}^{*} d_{A} \phi+L_{u}^{*} L_{u} \phi \\
d_{A}^{*} d_{A} \psi+L_{u}^{*} L_{u} \psi
\end{array}\right) .
$$

This proves (ii). To prove (iii) suppose that $(u, A)$ is a regular solution of (1), $J$ is integrable, and $\mathcal{D}_{u, A}^{*}(\eta, \phi, \psi)=0$. Then, by (ii), $\phi=\psi=0$ and $D_{u, A}^{*} \eta=0, L_{u}^{*} \eta=0$. Since $L_{u(p)}^{*}$ is injective for some $p \in P$ it follows that $\eta$ vanishes on some open set. Hence, by unique continuation, $\eta \equiv 0$. Thus we have proved that $\mathcal{D}_{u, A}^{*}$ is injective. Hence $\mathcal{D}_{u, A}$ has a dense range and hence, by elliptic regularity, $\mathcal{D}_{u, A}$ is onto. To prove (iv) note that, by Remark 4.5 , The operator $D_{u, A}$ is complex linear whenever $H=0, \dot{J}=0$, and $d_{A} u=0$. Hence $\mathcal{D}_{u, A}^{*}(\eta, \phi, \psi)=0$ implies $\phi=\psi=0$ and $D_{u, A}^{*} \eta=0, L_{u}^{*} \eta=0$. Since $\eta$ is a $(0,1)$-form we have

$$
d \mu(u) \eta=-L_{u}^{*} J \eta=L_{u}^{*}\left(\eta \circ J_{\Sigma}\right)=0 .
$$


Since $d_{A} u=0$ the image of $u$ is an orbit of some point $x_{0} \in M$ under the G-action. Since $(u, A)$ is irreducible, $\mathrm{G}_{x_{0}}=\{1\}$. Hence $\eta$ defines an element of the cokernel of the Cauchy-Riemann operator along the constant function $\bar{u} \equiv\left[x_{0}\right]: \Sigma \rightarrow \bar{M}=M / / \mathrm{G}(\tau)$. Since $\Sigma=S^{2}$, it follows from the Riemann-Roch theorem, that $\eta=0$.

\subsection{Transversality.}

In this section we establish transversality for the irreducible solutions of (3) for generic Hamiltonian perturbations.

Definition 4.9. A pair $(J, H) \in \mathcal{J} \times \mathcal{H}$ is called regular (for the sixtuple $(B, \Sigma, M, \omega, \mu, \tau))$ if the operator $\mathcal{D}_{u, A}$ is surjective for every $(u, A) \in$ $\widetilde{\mathcal{M}}_{B, \Sigma}^{*}(M, \omega, \mu, \tau ; J, H)$, i.e. for every irreducible solution of (3) representing the class $B$.

Given an almost complex structure $J \in \mathcal{J}$ we denote by $\mathcal{H}_{\text {reg }}(\tau, J) \subset \mathcal{H}$ the set of Hamiltonian perturbations $H$ such that the pair $(J, H)$ is regular. Given $\tau \in Z(\mathfrak{g})$ and $B \in H_{2}\left(M_{\mathrm{G}} ; \mathbb{Z}\right)$ we denote by $\mathcal{J}_{\text {reg }}(\tau, B) \subset \mathcal{J}$ the set of almost complex structures $J$ such that the pair $(J, H=0)$ is regular for $B$. If $(J, H)$ is regular then it follows from Proposition 4.6 and the infinite dimensional implicit function theorem that $\mathcal{M}_{B, \Sigma}^{*}(M, \omega, \mu, \tau ; J, H)$ is a finite dimensional smooth manifold of dimension

$$
\operatorname{dim} \mathcal{M}_{B, \Sigma}^{*}(M, \omega, \mu, \tau ; J, H)=(n-\operatorname{dim} \mathrm{G}) \chi(\Sigma)+2\left\langle c_{1}^{\mathrm{G}}(T M), B\right\rangle .
$$

\section{Theorem 4.10.}

(i) For every $J \in \mathcal{J}$ and every $\tau \in Z(\mathfrak{g})$ the set $\mathcal{H}_{\mathrm{reg}}(\tau, J)$ is a countable intersection of open and dense subsets of $\mathcal{H}$.

(ii) Assume $B$ is not a torsion class. Then, for every $\tau \in Z(\mathfrak{g})$, the set $\mathcal{J}_{\text {reg }}(\tau, B)$ is a countable intersection of open and dense subsets of $\mathcal{J}$.

Proof. Fix a sufficiently large integer $\ell$, a constant $c>0$, a compact set $K \subset M$, and a real number $p>2$. Consider the space of pairs $(u, A) \in$ $W_{\mathrm{G}}^{1, p}(P, M) \times \mathcal{A}^{1, p}(P)$ that satisfy

$$
u(P) \subset K, \quad\left\|d_{A} u\right\|_{L^{\infty}} \leq c
$$


and, for some $p_{0} \in P$ and all $\eta_{1}, \eta_{2} \in \mathfrak{g}$,

$$
\inf _{\mathrm{G}_{x} \neq\{\mathbb{1 1}\}}\left|u\left(p_{0}\right)-x\right| \geq \frac{1}{c}, \quad\left|\eta_{1}\right|+\left|\eta_{2}\right| \leq c\left|L_{u\left(p_{0}\right)} \eta_{1}+J L_{u\left(p_{0}\right)} \eta_{2}\right| .
$$

Denote

$$
\widetilde{\mathcal{M}}^{c, K}(H):=\left\{(u, A) \in \widetilde{\mathcal{M}}_{B, \Sigma}(M, \omega, \mu, \tau ; J, H) \mid u \text { and } A \text { satisfy (28-29) }\right\} .
$$

By Theorem 3.2, the moduli space

$$
\mathcal{M}^{c, K}(H):=\widetilde{\mathcal{M}}^{c, K}(H) / \mathcal{G}^{2, p}(P)
$$

is compact, and it consists entirely of irreducible solutions of (3). We shall examine the universal moduli space

$$
\begin{gathered}
\mathcal{U}^{\ell, c, K}:=\tilde{\mathcal{U}}^{\ell, c, K} / \mathcal{G}^{2, p}(P), \\
\tilde{\mathcal{U}}^{\ell, c, K}:=\left\{(u, A, H) \mid H \in \mathcal{H}^{\ell},(u, A) \in \widetilde{\mathcal{M}}^{c, K}(H),(28-29)\right\} .
\end{gathered}
$$

We prove that $\mathcal{U}^{\ell, c, K}$ is a Banach manifold. To see this we must show that the linearized operator

$$
\mathcal{D}_{u, A, H}: \mathcal{B}_{(u, A)}^{1, p} \oplus \mathcal{H}^{\ell} \rightarrow \mathcal{E}_{u}^{p} \oplus L^{p}\left(\Sigma, \mathfrak{g}_{P}\right)
$$

given by

$$
\mathcal{D}_{u, A, H}\left(\begin{array}{c}
\xi \\
\alpha \\
\hat{H}
\end{array}\right)=\mathcal{D}_{u, A}\left(\begin{array}{c}
\xi \\
\alpha
\end{array}\right)+\left(\begin{array}{c}
\left(X_{\hat{H}}(u)\right)^{0,1} \\
0 \\
0
\end{array}\right)
$$

is surjective for every triple $(u, A, H) \in \widetilde{\mathcal{U}}^{\ell, c, K}$. The proof of Theorem 3.1 shows that we may assume, without loss of generality, that $u$ and $A$ are of class $W^{\ell, p}$. By Proposition 4.6, $\mathcal{D}_{u, A}$ is a Fredholm operator and hence it suffices to prove that $\mathcal{D}_{u, A, H}$ has a dense range. Let $1 / p+1 / q=1$ and assume that the triple

$$
(\eta, \phi, \psi) \in L^{q}\left(\Sigma, \Lambda_{J_{u}}^{0,1} T^{*} \Sigma \otimes E_{u}\right) \times L^{q}\left(\Sigma, \mathfrak{g}_{P}\right) \times L^{q}\left(\Sigma, \mathfrak{g}_{P}\right)
$$

is $L^{2}$ orthogonal to the image of $\mathcal{D}_{u, A, H}$. Then, in particular, the triple $(\eta, \phi, \psi)$ is $L^{2}$-orthogonal to the image of $\mathcal{D}_{u, A}$. Since $u$ and $A$ are of class $W^{\ell, p}$ and $H$ is of class $C^{\ell}$ it follows from elliptic regularity that $\eta, \phi$, and $\psi$ are of class $W^{\ell, p}$ (and hence of class $C^{\ell-1}$ ) and

$$
\mathcal{D}_{u, A}^{*}(\eta, \phi, \psi)=0 \text {. }
$$


Moreover,

$$
\int_{\Sigma}\left\langle\eta, X_{\hat{H}}(u)\right\rangle \mathrm{dvol}_{\Sigma}=0
$$

for every $\hat{H} \in \mathcal{H}^{\ell}$. Since $u$ is irreducible, there exists a $p_{0} \in P$ such that

$$
\mathrm{G}_{u\left(p_{0}\right)}=\{\mathbb{1}\}, \quad \operatorname{im} L_{u\left(p_{0}\right)} \cap J L_{u\left(p_{0}\right)}=\{0\} .
$$

We prove that the linear map $\eta_{p_{0}}: T_{p_{0}} P \rightarrow T_{u\left(p_{0}\right)} M$ is equal to zero. Suppose otherwise that $\eta_{p_{0}} \neq 0$ and choose $v_{0} \in T_{p_{0}} P$ such that $\eta_{p_{0}}\left(v_{0}\right) \neq 0$. Since the linear map $\eta_{p_{0}}: T_{p_{0}} P \rightarrow T_{u\left(p_{0}\right)} M$ vanishes on $p_{0} \cdot \mathfrak{g}$ we have $v_{0} \notin p_{0} \cdot \mathfrak{g}$. Denote

$$
x_{0}:=u\left(p_{0}\right), \quad z_{0}:=\pi\left(p_{0}\right), \quad \zeta_{0}:=d \pi\left(p_{0}\right) v_{0} \neq 0,
$$

and choose $v_{1} \in T_{p_{0}} P$ such that

$$
\zeta_{1}:=d \pi\left(p_{0}\right) v_{1}=-J_{\Sigma} \zeta_{0}, \quad \eta_{p_{0}}\left(v_{1}\right)=J\left(z_{0}, x_{0}\right) \eta_{p_{0}}\left(v_{0}\right) .
$$

The last identity follows from the fact that $\eta$ is a $(0,1)$-form. By $(33), \eta_{p_{0}}\left(v_{0}\right)$ and $\eta_{p_{0}}\left(v_{1}\right)$ cannot both lie in the image of the map $L_{x_{0}}: \mathfrak{g} \rightarrow T_{x_{0}} M$. Let us assume, without loss of generality, that

$$
\eta_{p_{0}}\left(v_{1}\right) \notin \operatorname{im} L_{x_{0}} .
$$

Since $\mathrm{G}_{x_{0}}=\{\mathbb{1}\}$ there exists a G-invariant neighbourhood $U_{0} \subset M$ of $x_{0}$ such that $\mathrm{G}_{x}=\{\mathbb{1}\}$ for every $x \in U_{0}$. Since $\mathrm{G}_{x}=\{\mathbb{1}\}$ for every $x \in U_{0}$ there exists a smooth G-invariant function $\hat{H}_{0}: M \rightarrow \mathbb{R}$, supported in $U_{0}$, such that

$$
d \hat{H}_{0}\left(x_{0}\right) \eta_{p_{0}}\left(v_{1}\right)>0
$$

Hence

$$
\begin{aligned}
\left\langle X_{\hat{H}_{0}}\left(u\left(p_{0}\right)\right), \eta_{p_{0}}\left(v_{0}\right)\right\rangle & =\omega\left(X_{\hat{H}_{0}}\left(x_{0}\right), J\left(z_{0}, x_{0}\right) \eta_{p_{0}}\left(v_{0}\right)\right) \\
& =\omega\left(X_{\hat{H}_{0}}\left(x_{0}\right), \eta_{p_{0}}\left(v_{1}\right)\right) \\
& =d \hat{H}_{0}\left(x_{0}\right) \eta_{p_{0}}\left(v_{1}\right) \\
& >0 .
\end{aligned}
$$

Now let $\zeta: \Sigma \rightarrow T \Sigma$ be a vector field such that $\zeta\left(z_{0}\right)=\zeta_{0}$. Choose a neighbourhood $V_{0}$ of $z_{0}$ such that, for every $p \in P$ and every $v \in T_{p} P$,

$$
\pi(p) \in V_{0}, \quad d \pi(p) v=\zeta(\pi(p)) \quad \Longrightarrow \quad\left\langle X_{\hat{H}_{0}}(u(p)), \eta_{p}(v)\right\rangle>0
$$


Now choose a cutoff function $\beta: \Sigma \rightarrow[0,1]$ which is supported in $V_{0}$ and satisfies $\beta\left(z_{0}\right)=1$. Define $\hat{H} \in \mathcal{H}$ by the conditions

$$
\hat{H}_{\zeta(z)}=\beta(z) \hat{H}_{0}, \quad \hat{H}_{J_{\Sigma} \zeta(z)}=0 .
$$

Then $\left\langle\eta, X_{\hat{H}}(u)\right\rangle>0$ at the point $z_{0}$ and $\left\langle\eta, X_{\hat{H}}(u)\right\rangle \geq 0$ everywhere. Hence

$$
\int_{\Sigma}\left\langle\eta, X_{\hat{H}}(u)\right\rangle \operatorname{dvol}_{\Sigma}>0
$$

in contradiction to (32). Thus we have proved that, for every $p \in P$,

$$
\mathrm{G}_{u(p)}=\{\mathbb{1}\} \quad \Longrightarrow \quad \eta(p)=0 .
$$

Moreover, by (31) and Proposition 4.8, we have that $\phi=0$. Since $\operatorname{ker} L_{u(p)}=$ 0 whenever $\mathrm{G}_{u(p)}=\{11\}$, it follows from (24) and (32) that

$$
\mathrm{G}_{u(p)}=\{\mathbb{1}\} \quad \Longrightarrow \quad \psi(p)=0 .
$$

Hence $\eta$ and $\psi$ vanish simultaneously on some open subset of $P$. Since $\mathcal{D}_{u, A}^{*}(\eta, 0, \psi)=0$ it follows by unique continuation for first order elliptic operators that $\eta=0$ and $\psi=0$.

Thus we have proved that the operator $\mathcal{D}_{u, A, H}$ has a dense range for every $(u, A, H) \in \widetilde{\mathcal{U}}^{\ell, c, K}$, as claimed. Since $\mathcal{D}_{u, A}$ is Fredholm, the operator $\mathcal{D}_{u, A, H}$ has a right inverse. Hence, by the implicit function theorem, $\mathcal{U}^{\ell, c, K}$ is a separable Banach manifold of class $C^{\ell-1}$. The projection

$$
\pi^{\ell, c, K}: \mathcal{U}^{\ell, c, K} \rightarrow \mathcal{H}^{\ell}
$$

is a Fredholm map of index $(n-\operatorname{dim} \mathrm{G}) \chi(\Sigma)+2\left\langle c_{1}^{\mathrm{G}}(T M), B\right\rangle$. Hence, for $\ell$ sufficiently large, it follows from the Sard-Smale theorem, that the set $\mathcal{H}_{\text {reg }}^{\ell, c, K}(\tau, J) \subset \mathcal{H}^{\ell}$ of regular values of $\pi^{\ell, c, K}$ is dense in $\mathcal{H}^{\ell}$. Moreover, the moduli space

$$
\mathcal{M}^{\ell, c, K}(H):=\left\{[u, A] \mid([u, A], H) \in \mathcal{U}^{\ell, c, K}\right\}
$$

is compact for every $H$. Hence the set $\mathcal{H}_{\text {reg }}^{\ell, c, K}(\tau, J)$ is open and dense in $\mathcal{H}^{\ell}$. Hence the set $\mathcal{H}_{\text {reg }}^{c, K}(\tau, J)=\mathcal{H}_{\text {reg }}^{\ell, c, K}(\tau, J) \cap \mathcal{H}$ is dense in $\mathcal{H}^{\ell}$ and hence is also dense in $\mathcal{H}$. That it is open follows again from compactness. Hence the set

$$
\mathcal{H}_{\text {reg }}(\tau, J)=\bigcap_{c, K} \mathcal{H}_{\text {reg }}^{c, K}(\tau, J)
$$


is a countable intersection of open and dense subsets of $\mathcal{H}$. This proves (i).

We sketch the proof of (ii). Assume $B$ is not a torsion class. Then, by Lemma 2.3, every solution $(u, A)$ of $(3)$ with $H=0$ representing the class $B$ satisfies $d_{A} u \not \equiv 0$ and so, by Lemma $2.5, d_{A} u \neq 0$ almost everywhere. Hence, for every irreducible solution $(u, A)$ of $(3)$ with $H=0$ that represents the class $B$, there exists a point $p_{0} \in P$ such that

$$
\mathrm{G}_{u\left(p_{0}\right)}=\{\mathbb{1}\}, \quad \operatorname{im} L_{u\left(p_{0}\right)} \cap \operatorname{im} J L_{u\left(p_{0}\right)}=\{0\}, \quad d_{A}\left(u\left(p_{0}\right)\right) \neq 0 .
$$

With this understood the proof of assertion (ii) is almost word by word the same as that of (i) and will be omitted.

\subsection{Cobordisms.}

Let $\left(\tau_{\lambda}, J_{\lambda}\right) \in Z(\mathfrak{g}) \times \mathcal{J}$ and $H_{\lambda} \in \mathcal{H}_{\text {reg }}\left(\tau_{\lambda}, J_{\lambda}\right)$ for $\lambda=0,1$. For every smooth homotopy

$$
\left\{\tau_{\lambda}, J_{\lambda}, H_{\lambda}\right\}_{0 \leq \lambda \leq 1} \in Z(\mathfrak{g}) \times \mathcal{J} \times \mathcal{H}
$$

from $\left(\tau_{0}, J_{0}, H_{0}\right)$ to $\left(\tau_{1}, J_{1}, H_{1}\right)$ we consider the space

$$
\mathcal{W}_{B, \Sigma}^{*}:=\mathcal{W}_{B, \Sigma}^{*}\left(\left\{\tau_{\lambda}, J_{\lambda}, H_{\lambda}\right\}_{\lambda}\right):=\bigcup_{0 \leq \lambda \leq 1}\{\lambda\} \cup \mathcal{M}_{B, \Sigma}^{*}\left(\tau_{\lambda} ; J_{\lambda}, H_{\lambda}\right)
$$

Definition 4.11. A homotopy $\left\{\tau_{\lambda}, J_{\lambda}, H_{\lambda}\right\}_{\lambda}$ is called regular (for the tuple $(B, \Sigma, M, \omega, \mu))$ if $H_{\lambda} \in \mathcal{H}_{\mathrm{reg}}\left(\tau_{\lambda}, J_{\lambda}\right)$ for $\lambda=0,1$ and, for every triple $(\lambda,[u, A]) \in \mathcal{W}_{B, \Sigma}^{*}$, we have

$$
\mathcal{E}_{u}=\operatorname{im} \mathcal{D}_{u, A}+\operatorname{span}^{\mathbb{R}} \zeta_{\lambda, u, A},
$$

where

$$
\zeta_{\lambda, u, A}:=\left(\begin{array}{c}
\nabla_{\lambda}\left(\bar{\partial}_{J_{\lambda}, H_{\lambda}, A}(u)\right) \\
0 \\
-\partial_{\lambda} \tau_{\lambda}
\end{array}\right)
$$

Here the expression $\nabla_{\lambda}\left(\bar{\partial}_{J_{\lambda}, H_{\lambda}, A}(u)\right)$ is independent of the (Hermitian) connection used to define it. If $\left\{\tau_{\lambda}, J_{\lambda}, H_{\lambda}\right\}_{\lambda}$ is a regular homotopy then the moduli space $\mathcal{W}_{B, \Sigma}^{*}\left(\left\{\tau_{\lambda}, J_{\lambda}, H_{\lambda}\right\}_{\lambda}\right)$ is a smooth finite dimensional manifold with boundary

$\partial \mathcal{W}_{B, \Sigma}^{*}\left(\left\{\tau_{\lambda}, J_{\lambda}, H_{\lambda}\right\}_{\lambda}\right)=\{0\} \times \mathcal{M}_{B, \Sigma}^{*}\left(\tau_{0} ; J_{0}, H_{0}\right) \cup\{1\} \times \mathcal{M}_{B, \Sigma}^{*}\left(\tau_{1} ; J_{1}, H_{1}\right)$. 
Let $\mathcal{H}^{[0,1]}\left(H_{0}, H_{1}\right)$ denote the space of smooth paths $[0,1] \rightarrow \mathcal{H}: \lambda \mapsto H_{\lambda}$ with fixed endpoints $H_{0}$ and $H_{1}$. Given a homotopy $\left\{\tau_{\lambda}, J_{\lambda}\right\}_{\lambda}$ from $\left(\tau_{0}, J_{0}\right)$ to $\left(\tau_{1}, J_{1}\right)$ denote by $\mathcal{H}_{\text {reg }}^{[0,1]}\left(H_{0}, H_{1} ;\left\{\tau_{\lambda}, J_{\lambda}\right\}_{\lambda}\right) \subset \mathcal{H}^{[0,1]}\left(H_{0}, H_{1}\right)$ the set of all smooth homotopies $\left\{H_{\lambda}\right\}_{\lambda}$ from $H_{0}$ to $H_{1}$ such that the triple $\left\{\tau_{\lambda}, J_{\lambda}, H_{\lambda}\right\}_{\lambda}$ is regular. The next theorem asserts the set of regular homotopies is of the second category in the sense of Baire.

Theorem 4.12. Assume $\mathrm{G}=T$ is a torus. Let $\left\{\tau_{\lambda}, J_{\lambda}\right\}_{0 \leq \lambda \leq 1}$ be a smooth homotopy in $C^{\infty}(\Sigma, Z(\mathfrak{g})) \times \mathcal{J}$ and let $H_{\lambda} \in \mathcal{H}_{\mathrm{reg}}\left(\tau_{\lambda}, J_{\lambda}\right)$ for $\lambda=0,1$. Then the set $\mathcal{H}_{\mathrm{reg}}^{[0,1]}\left(H_{0}, H_{1} ;\left\{\tau_{\lambda}, J_{\lambda}\right\}_{\lambda}\right)$ is a countable intersection of open and dense subsets of $\mathcal{H}^{[0,1]}\left(H_{0}, H_{1}\right)$.

Proof. The proof is similar to that of Theorem 4.10 and we only sketch the main points. Denote by $\hat{\mathcal{H}}^{\ell}$ the set of all $C^{\ell}$ homotopies from $H_{0}$ to $H_{1}$. Fix a constant $c>0$ and a compact set $K \subset M$ and consider the universal moduli space of all gauge equivalence classes of quadruples

$$
\left(\lambda, u, A,\left\{H_{\lambda}\right\}_{\lambda}\right) \in[0,1] \times W_{\mathrm{G}}^{1, p}(P, M) \times \mathcal{A}^{1, p}(P) \times \hat{\mathcal{H}}^{\ell}
$$

such that $(u, A) \in \widetilde{\mathcal{M}}_{B, \Sigma}^{*}\left(\tau_{\lambda} ; J_{\lambda}, H_{\lambda}\right)$ and $u$ and $A$ satisfy (28-29). The proof of Theorem 4.10 shows that this space is a separable Banach manifold. The projection

$$
\left(\lambda,[u, A],\left\{H_{\lambda}\right\}_{\lambda}\right) \mapsto\left\{H_{\lambda}\right\}_{\lambda}
$$

is then a Fredholm map and a smooth homotopy $\left\{H_{\lambda}\right\}_{\lambda}$ is a regular value of this projection for every triple $(K, c, \ell)$ if and only if

$$
\left\{H_{\lambda}\right\}_{\lambda} \in \mathcal{H}_{\mathrm{reg}}^{[0,1]}\left(H_{0}, H_{1} ;\left\{\tau_{\lambda}, J_{\lambda}\right\}_{\lambda}\right) .
$$

Hence the result follows from the Sard-Smale theorem.

\subsection{Orientation.}

In this subsection we shall prove that the moduli spaces $\mathcal{M}_{B, \Sigma}^{*}(M, \omega, \mu ; J, H)$ carry natural orientations. Consider the determinant line bundle

$$
\operatorname{det}(\mathcal{D}) \rightarrow \mathcal{B}=C_{\mathrm{G}}^{\infty}(P, M) \times \mathcal{A}(P)
$$

whose fibre over $(u, A)$ is the 1-dimensional real vector space

$$
\operatorname{det}\left(\mathcal{D}_{u, A}\right):=\Lambda^{\max }\left(\operatorname{ker} \mathcal{D}_{u, A}\right) \otimes \Lambda^{\max }\left(\operatorname{ker} \mathcal{D}_{u, A}^{*}\right)
$$


(See, for example, [31, Appendix A] for a detailed exposition.) Here

$$
\mathcal{D}_{u, A}=\mathcal{D}_{u, A}: T_{(u, A)} \mathcal{B} \rightarrow \mathcal{E}_{u}
$$

denotes the Fredholm operator given by equation (23). The orientation of the moduli spaces is an immediate consequence of the following proposition. The proof is reminiscent of the arguments in [23, 31].

Proposition 4.13. The determinant line bundle $\operatorname{det}(\mathcal{D}) \rightarrow \mathcal{B}$ admits a natural $\mathcal{G}$-invariant orientation.

Proof. The tangent space

$$
T_{(u, A)} \mathcal{B}=C^{\infty}\left(\Sigma, E_{u}\right) \oplus \Omega^{1}\left(\Sigma, \mathfrak{g}_{P}\right)
$$

admits a natural complex structure given by the complex structure $J_{u}$ on $E_{u}$ and the Hodge $*$-operator on $\Omega^{1}(\Sigma)$. The fibre

$$
\mathcal{E}_{u}=\Omega_{J_{u}}^{0,1}\left(\Sigma, E_{u}\right) \oplus \Omega^{0}\left(\Sigma, \mathfrak{g}_{P}\right) \oplus \Omega^{0}\left(\Sigma, \mathfrak{g}_{P}\right)
$$

also admits a complex structure given by the complex structure $J_{u}$ on $E_{u}$ and by the map $(\phi, \psi) \mapsto(-\psi, \phi)$ on $\Omega^{0}\left(\Sigma, \mathfrak{g}_{P}\right) \oplus \Omega^{0}\left(\Sigma, \mathfrak{g}_{P}\right)$. The only term in the formula $(23)$ for the operator $\mathcal{D}_{u, A}$ that is not necessarily complex linear, is the operator

$$
D_{u, A}: C^{\infty}\left(\Sigma, E_{u}\right) \rightarrow \Omega_{J_{u}}^{0,1}\left(\Sigma, E_{u}\right)
$$

However, by Remark 4.5, the complex anti-linear part of $D_{u, A}$ is of zeroth order and is therefore compact. Hence $\mathcal{D}_{u, A}$ is a compact perturbation of a complex linear operator and hence admits a natural orientation. It follows that the real line bundle $\operatorname{det}(\mathcal{D}) \rightarrow \mathcal{B}$ admits a natural orientation (see [23]).

Now let $g \in \mathcal{G}(P)$ and choose a pair $(u, A) \in \mathcal{B}$. Linearizing the action of the gauge group gives rise to isomorphisms

$$
\begin{aligned}
& \operatorname{ker} \mathcal{D}_{u, A} \rightarrow \operatorname{ker} \mathcal{D}_{g^{-1} u, g^{*} A}:(\xi, \alpha) \mapsto\left(g^{-1} \xi, g^{-1} \alpha g\right), \\
& \operatorname{ker} \mathcal{D}_{u, A}^{*} \rightarrow \operatorname{ker} \mathcal{D}_{g^{-1} u, g^{*} A}^{*}:(\eta, \phi, \psi) \mapsto\left(g^{-1} \eta, g^{-1} \phi g, g^{-1} \psi g\right) .
\end{aligned}
$$

We prove that the resulting isomorphism of determinant lines is orientation preserving with respect to the natural orientations introduced above. To see this, we assume first that $H=0$ and $J(z, \cdot)$ is integrable near $u(p)$ for every $z \in \Sigma$ and every $p \in P$ with $\pi(p)=z$. Then the operators $\mathcal{D}_{u, A}$ and 
$\mathcal{D}_{g^{-1} u, g^{*} A}$ are both complex linear, and hence the orientations of $\operatorname{det}\left(\mathcal{D}_{u, A}\right)$ and $\operatorname{det}\left(\mathcal{D}_{g^{-1} u, g^{*} A}\right)$ both agree with the orientations induced by the complex structures. Hence, in this case, the result follows from the fact that the maps (35) are complex linear. In general, the result follows from the fact that the spaces $\mathcal{H}$ and $\mathcal{J}$ are both connected and hence the isomorphism $\operatorname{det}\left(\mathcal{D}_{u, A}\right) \rightarrow \operatorname{det}\left(\mathcal{D}_{g^{-1} u, g^{*} A}\right)$ is orientation preserving for some pair $(H, J)$ if and only if it is orientation preserving for every pair $(H, J)$.

Proposition 4.14. Let $\left\{H_{\lambda}\right\}_{\lambda} \in \mathcal{H}_{\mathrm{reg}}^{[0,1]}\left(H_{0}, H_{1} ;\left\{\tau_{\lambda}, J_{\lambda}\right\}_{\lambda}\right)$. Then the moduli space $\mathcal{W}_{B, \Sigma}^{*}\left(\left\{\tau_{\lambda}, J_{\lambda}, H_{\lambda}\right\}_{\lambda}\right.$ is an oriented cobordism from $\mathcal{M}_{B, \Sigma}^{*}\left(\tau_{0} ; J_{0}, H_{0}\right)$ to $\mathcal{M}_{B, \Sigma}^{*}\left(\tau_{1} ; J_{1}, H_{1}\right)$.

Proof. The tangent space of $\mathcal{W}_{B, \Sigma}^{*}$ at a triple $(\lambda, u, A)$ is the kernel of the operator

$$
\hat{\mathcal{D}}_{\lambda, u, A}: \mathbb{R} \times T_{(u, A)} \mathcal{B} \rightarrow \mathcal{E}_{u}
$$

given by

$$
\hat{\mathcal{D}}_{\lambda, u, A}(\hat{\lambda}, \xi, \alpha):=\mathcal{D}_{u, A}(\xi, \alpha)+\hat{\lambda} \zeta_{\lambda, u, A}
$$

for $\hat{\lambda} \in \mathbb{R}$ and $(\xi, \alpha) \in T_{(u, A)} \mathcal{B}$, where $\zeta_{\lambda, u, A} \in \mathcal{E}_{u}$ is given by (34). Since $\mathcal{D}_{\lambda, u, A}$ is surjective, the orientation of the kernel is determined by the orientation of the determinant line. Thus we must examine the determinant line bundle

$$
\operatorname{det}(\hat{\mathcal{D}}) \rightarrow[0,1] \times \mathcal{B}
$$

whose fibre over a triple $(\lambda, u, A) \in[0,1] \times \mathcal{B}$ is $\operatorname{det}\left(\mathcal{D}_{\lambda, u, A}\right)$. The homotopy $t \mapsto t \zeta_{\lambda, u, A}$ yields a natural isomorphism

$$
\operatorname{det}(\hat{\mathcal{D}}) \cong \operatorname{pr}_{1}^{*} T[0,1] \otimes \operatorname{pr}_{2}^{*} \operatorname{det}(\mathcal{D}) \cong \operatorname{pr}_{2}^{*} \operatorname{det}(\mathcal{D}),
$$

where $\operatorname{pr}_{1}:[0,1] \times \mathcal{B} \rightarrow[0,1]$ and $\operatorname{pr}_{2}:[0,1] \times \mathcal{B} \rightarrow \mathcal{B}$ denote the obvious projections. This is because the tangent space $T_{\lambda}[0,1]$ is canonically isomorphic to $\mathbb{R}$ and, for $t=0$, we have

$$
\operatorname{ker} \hat{\mathcal{D}} \cong \mathbb{R} \times \operatorname{ker} \mathcal{D}, \quad \text { coker } \hat{\mathcal{D}} \cong \operatorname{coker} \mathcal{D} .
$$

Hence $\operatorname{det}(\hat{\mathcal{D}})$ inherits the orientation of $\operatorname{det}(\mathcal{D})$ and, since the orientation of $\operatorname{det}(\mathcal{D})$ is invariant under the action of $\mathcal{G}$ so is the orientation of $\operatorname{det}(\hat{\mathcal{D}})$. It follows that the manifold $\mathcal{W}_{B, \Sigma}^{*}$ admits a natural orientation.

Now choose a triple $(\lambda,[u, A]) \in \mathcal{W}_{B, \Sigma}^{*}$ such that $\mathcal{D}_{u, A}$ is onto. Then a positively oriented basis of $T_{(\lambda,[u, A])} \mathcal{W}_{B, \Sigma}^{*}$ has the form

$$
\left(1, \xi_{0}, \alpha_{0}\right),\left(0, \xi_{1}, \alpha_{1}\right), \ldots,\left(0, \xi_{k}, \alpha_{k}\right),
$$


where the vectors $\left(\xi_{1}, \alpha_{1}\right), \ldots,\left(\xi_{k}, \alpha_{k}\right)$ form a positively oriented basis of the kernel of $\mathcal{D}_{u, A}$. With the standard convention for orienting the boundary (the outward unit normal vector comes first) the result follows.

\section{Integer invariants.}

Let $(M, \omega, \mu)$ be a symplectic manifold of (real) dimension $2 n$ equipped with a Hamiltonian action by a compact Lie group $\mathrm{G}$ which is generated by a moment map $\mu: M \rightarrow \mathfrak{g}$. Suppose that the triple $(M, \omega, \mu)$ satisfies $(H 1-3)$. We shall define rational invariants of the triple $(M, \omega, \mu)$ for central regular values of the moment map under these hypotheses. Conditions $(H 1-2)$ are needed to prove that the moduli spaces are compact. It should be possible to remove condition $(H 3)$, however, the construction of the invariants without this condition will probably require considerably more analysis than has been carried out in the present paper. This would include a full version of compactness for the solutions of (3) without loss of energy and with preservation of the homotopy class in the limit, as well as the construction of virtual moduli cycles analogous to the definition of the Gromov-Witten invariants for general symplectic manifolds as in $[13,20,22,29]$. On the other hand, there are many interesting examples that satisfy $(H 1-3)$, such as linear actions on complex vector spaces. (In this case $(H 1)$ implies $(H 2)$, and (H3) is obvious.) In the present section we define integer invariants under the additional assumption that $\mathrm{G}$ acts freely on $\mu^{-1}(\tau)$ for some central element $\tau \in Z(\mathfrak{g})$. This hypothesis will in Section 7 be replaced by the assumption that $\mu$ has a central regular value.

\subsection{Smooth moduli spaces.}

Fix an equivariant homology class $B \in H_{2}\left(M_{\mathrm{G}} ; \mathbb{Z}\right)$ and a compact Riemann surface $\left(\Sigma, J_{\Sigma}, \operatorname{dvol}_{\Sigma}\right)$. Recall from Section 4 the definition of the moduli space

$$
\mathcal{M}(\tau ; J, H)=\mathcal{M}_{B, \Sigma}(\tau ; J, H)=\widetilde{\mathcal{M}}_{B, \Sigma}(\tau ; J, H) / \mathcal{G}(P)
$$

of gauge equivalence classes of solutions of (3) for a (family of) almost complex structures $J \in \mathcal{J}$ and a Hamiltonian perturbation $H \in \mathcal{H}$. Consider the set

$$
Z_{0}(\mathfrak{g}):=\left\{\tau \in Z(\mathfrak{g}) \mid \mathrm{G} \text { acts freely on } \mu^{-1}(\tau)\right\} .
$$

By $(H 1)$, this set is open and we assume here that it is nonempty. Choose a smooth function $\delta: Z_{0}(\mathfrak{g}) \rightarrow(0, \infty)$ such that

$$
|\mu(x)-\tau|^{2} \leq \delta(\tau) \quad \Longrightarrow \quad \mathrm{G}_{x}=\{1\}, \operatorname{im} L_{x} \cap \operatorname{im} J(z, x) L_{x}=\{0\}
$$


for all $(z, x) \in \Sigma \times M$ and $\tau \in Z_{0}(\mathfrak{g})$. If

$$
\frac{\langle[\omega+\tau-\mu], B\rangle+\left\|\Omega_{H}\right\|}{\operatorname{Vol}(\Sigma)} \leq \delta(\tau)
$$

then the moduli space $\mathcal{M}_{B, \Sigma}(\tau ; J, H)$ consists entirely of irreducible solutions (Lemma 4.2) and hence is a smooth manifold for a generic Hamiltonian function $H$ that satisfies (37) (Theorem 4.10). Moreover, $\mathcal{M}_{B, \Sigma}(\tau ; J, H)$ is compact whenever $H$ has compact support and $J$ agrees with the almost complex structure $J_{0}$ of hypothesis (H2) ouside of some compact subset of $M$ (Corollary 3.5 ). Let us denote by $\mathcal{J}_{0}$ the space of almost complex structures that agree with $J_{0}$ outside of a compact set, by $\mathcal{H}(\tau ; \delta)$ the space of compactly supported Hamiltonian perturbations that satisfy (37), and, for $\tau \in Z_{0}(\mathfrak{g})$ and $J \in \mathcal{J}_{0}$, denote by

$$
\mathcal{H}_{\mathrm{reg}}(\tau, J ; \delta):=\mathcal{H}_{\mathrm{reg}}(\tau, J) \cap \mathcal{H}(\tau ; \delta)
$$

the subset of regular perturbations in the sense of Definition 4.9. Let

$$
\mathcal{B}^{*} \subset \mathcal{B}=C_{\mathrm{G}}^{\infty}(P, M) \times \mathcal{A}(P)
$$

denote the subset of irreducible pairs $(u, A) \in \mathcal{B}$ (see Definition 4.1). By the local slice theorem (see Theorem B.1) the quotient space $\mathcal{B}^{*} / \mathcal{G}$ is an infinite dimensional Frêchet manifold (determined by $\mu, P$, and $\Sigma$ ). The next theorem summarizes our results about the moduli spaces $\mathcal{M}_{B, \Sigma}(\tau ; J, H)$.

Theorem 5.1. Assume $(H 1-3)$ and let $\tau \in Z_{0}(\mathfrak{g})$. Then the following holds.

(i) For every pair $(\tau, J) \in Z_{0}(\mathfrak{g}) \times \mathcal{J}_{0}$ the set $\mathcal{H}_{\text {reg }}(\tau, J ; \delta)$ is open and dense in $\mathcal{H}(\tau ; \delta)$.

(ii) For every pair $(\tau, J) \in Z_{0}(\mathfrak{g}) \times \mathcal{J}_{0}$ and every $H \in \mathcal{H}_{\mathrm{reg}}(\tau, J ; \delta)$ the moduli space $\mathcal{M}_{B, \Sigma}(\tau ; J, H)$ is a compact smooth naturally oriented submanifold of $\mathcal{B}^{*} / \mathcal{G}$ of dimension

$$
\operatorname{dim} \mathcal{M}_{B, \Sigma}(\tau ; J, H)=2 m:=(n-\operatorname{dim} \mathrm{G}) \chi(\Sigma)+2\left\langle c_{1}^{\mathrm{G}}(T M), B\right\rangle .
$$

(iii) For $\lambda=0,1$ let $\left(\tau_{\lambda}, J_{\lambda}\right) \in Z_{0}(\mathfrak{g}) \times \mathcal{J}_{0}$ and $H_{\lambda} \in \mathcal{H}_{\text {reg }}\left(\tau_{\lambda}, J_{\lambda} ; \delta\right)$. Suppose that $\tau_{0}$ and $\tau_{1}$ belong to the same component of $Z_{0}(\mathfrak{g})$. Then the moduli spaces $\mathcal{M}\left(\tau_{0} ; J_{0}, H_{0}\right)$ and $\mathcal{M}\left(\tau_{1} ; J_{1}, H_{1}\right)$ are oriented cobordant in $\mathcal{B}^{*} / \mathcal{G}$, i.e. there exists a compact oriented submanifold

$$
\mathcal{W} \subset[0,1] \times \mathcal{B}^{*} / \mathcal{G}
$$


of dimension $2 m+1$ such that

$$
\partial \mathcal{W}=\{1\} \times \mathcal{M}\left(\tau_{1} ; J_{1}, H_{1}\right)-\{0\} \times \mathcal{M}\left(\tau_{0} ; J_{0}, H_{0}\right) .
$$

Proof. By Corollary 3.5, the moduli space $\mathcal{M}(\tau ; J, H)$ is a compact subset of $\mathcal{B} / \mathcal{G}$ and, by Lemma 4.2 , it consists entirely of irreducible solutions of (3) for every $H \in \mathcal{H}(\tau ; \delta)$. Hence the set $\mathcal{H}_{\text {reg }}(\tau, J ; \delta)$ is open in $\mathcal{H}(\tau ; \delta)$. By Theorem 4.10, it is dense. This proves (i).

That $\mathcal{M}^{*}(\tau ; J, H)$ is a smooth submanifold of $\mathcal{B}^{*} / \mathcal{G}$ of dimension $2 m$ for $H \in \mathcal{H}_{\text {reg }}(\tau, J ; \delta)$ follows from the definitions, from Proposition 4.6, and from the implicit function theorem. That $\mathcal{M}(\tau ; J, H)=\mathcal{M}^{*}(\tau ; J, H)$ follows from Lemma 4.2, and that $\mathcal{M}(\tau ; J, H)$ is orientable follows from Proposition 4.13. This proves (ii). Assertion (iii) follows from Theorem 4.12, Corollary 3.5, and Proposition 4.14.

\subsection{Definition of the invariants.}

The evaluation map. The group $\mathcal{G} \times \mathrm{G}$ acts freely on the product $\mathcal{B}^{*} \times P$ by

$$
(g, h)^{*}(u, A, p):=\left(g^{-1} u, g^{*} A, p g(p)^{-1} h\right)
$$

for $g \in \mathcal{G}, h \in \mathrm{G},(u, A) \in \mathcal{B}^{*}$, and $p \in P$. Hence there is a principal G-bundle

$$
\mathcal{P}:=\left(\mathcal{B}^{*} \times P\right) / \mathcal{G} \longrightarrow \mathcal{B}^{*} / \mathcal{G} \times \Sigma .
$$

The classifying map $\mathcal{B}^{*} / \mathcal{G} \times \Sigma \rightarrow$ BG of this bundle lifts to a map $\theta$ : $\mathcal{B}^{*} \times P \rightarrow$ EG that is $\mathcal{G}$-invariant and G-equivariant:

$$
\theta\left(g^{-1} u, g^{*} A, p g(p)^{-1} h\right)=h^{-1} \theta(u, A, p) .
$$

Likewise, the evaluation map $\mathcal{B}^{*} \times P \rightarrow M:(u, A, p) \mapsto u(p)$ is $\mathcal{G}$-invariant and G-equivariant. These two maps together give rise to a map

$$
\mathrm{ev}_{\mathrm{G}}: \mathcal{B}^{*} / \mathcal{G} \times \Sigma \longrightarrow M_{\mathrm{G}}:=M \times_{\mathrm{G}} \mathrm{EG}
$$

given by

$$
\operatorname{ev}_{\mathrm{G}}([u, A, p]):=[u(p), \theta(u, A, p)] .
$$

The composition of $\mathrm{ev}_{\mathrm{G}}$ with the projection $\rho_{M}: M_{\mathrm{G}} \rightarrow \mathrm{BG}$ is the classifying map of $\mathcal{P}$ :

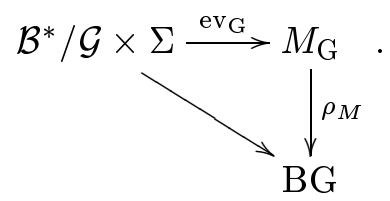


If $M$ is contractible, then the projection $\rho_{M}: M_{\mathrm{G}} \rightarrow \mathrm{BG}$ is a homotopy equivalence.

The projection. Now fix a point $p_{0} \in P$, denote by $\mathcal{G}_{0}:=\left\{g \in \mathcal{G} \mid g\left(p_{0}\right)=\right.$ id \} the based gauge group, and consider the space

$$
\mathcal{A}_{\mathcal{G}}:=\mathcal{A} \times_{\mathcal{G}} \mathrm{EG}
$$

where $\mathcal{G}$ acts by $g^{*}(A, e):=\left(g^{*} A, g\left(p_{0}\right)^{-1} e\right)$. This space can be identified with $\mathcal{A} / \mathcal{G}_{0} \times{ }_{\mathrm{G}}$ EG. Since $\mathcal{G}$ acts freely on $\mathcal{B}^{*}$ there is a principal G-bundle

$$
\mathcal{P}_{0}:=\mathcal{B}^{*} / \mathcal{G}_{0} \longrightarrow \mathcal{B}^{*} / \mathcal{G}
$$

The classifying map of this bundle lifts to a $\mathcal{G}$-equivariant map $\theta_{0}: \mathcal{B}^{*} \rightarrow \mathrm{EG}$, which is equal to the restriction of $\theta$ to $\mathcal{B}^{*} \times\left\{p_{0}\right\}$. It satisfies

$$
\theta_{0}\left(g^{-1} u, g^{*} A\right)=g\left(p_{0}\right)^{-1} \theta_{0}(u, A)
$$

and gives rise to a projection

$$
\pi_{\mathcal{A}}: \mathcal{B}^{*} / \mathcal{G} \rightarrow \mathcal{A}_{\mathcal{G}}
$$

given by

$$
\pi_{\mathcal{A}}([u, A]):=\left[A, \theta_{0}(u, A)\right] .
$$

The composition of $\pi_{\mathcal{A}}$ with the projection $\rho_{\mathcal{A}}: \mathcal{A}_{\mathcal{G}} \rightarrow$ BG is the classifying map of $\mathcal{P}_{0}$ :

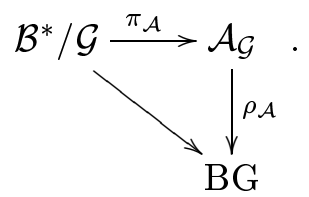

The invariants. We define invariants of the sixtuple $(M, \omega, \mu, \tau, B, \Sigma)$ with $\tau \in Z_{0}(\mathfrak{g})$ by integrating suitable cohomology classes over the moduli space $\mathcal{M}_{B, \Sigma}(\tau ; J, H)$. Such cohomology classes can be obtained by pulling back equivariant cohomology classes on $M$ under the evaluation map ev $\mathrm{G}_{\mathrm{G}}$ and equivariant cohomology classes on $\mathcal{A} / \mathcal{G}_{0}$ under the projection $\pi_{\mathcal{A}}$. Let $\alpha \in H^{*}\left(\mathcal{A}_{\mathcal{G}} ; \mathbb{Z}\right), \beta_{1}, \ldots, \beta_{k} \in H^{*}\left(M_{\mathrm{G}} ; \mathbb{Z}\right)$, and $\gamma_{1}, \ldots, \gamma_{k} \in H_{*}(\Sigma ; \mathbb{Z})$ such that

$$
\operatorname{deg}(\alpha)+\sum_{i=1}^{k} \operatorname{deg}\left(\beta_{i}\right)-\sum_{i=1}^{k} \operatorname{deg}\left(\gamma_{i}\right)=2 m
$$


and define

$$
\begin{aligned}
& \Phi_{B, \Sigma}^{M, \mu-\tau}\left(\alpha ; \beta_{1}, \ldots, \beta_{k} ; \gamma_{1}, \ldots, \gamma_{k}\right) \\
& :=\int_{\mathcal{M}_{B, \Sigma}(\tau ; J, H)} \pi_{\mathcal{A}}^{*} \alpha \smile \mathrm{ev}_{\mathrm{G}}^{*} \beta_{1} / \gamma_{1} \smile \ldots \smile \mathrm{ev}_{\mathrm{G}}^{*} \beta_{k} / \gamma_{k} .
\end{aligned}
$$

Here the map $H^{q}\left(\mathcal{B}^{*} / \mathcal{G} \times \Sigma ; \mathbb{Z}\right) \times H_{i}(\Sigma ; \mathbb{Z}) \rightarrow H^{q-i}\left(\mathcal{B}^{*} / \mathcal{G} ; \mathbb{Z}\right):(\beta, \gamma) \mapsto \beta / \gamma$ denotes the slant product, $J \in \mathcal{J}_{0}$, and $H \in \mathcal{H}_{\text {reg }}(\tau, J ; \delta)$, where the function $\delta: Z_{0}(\mathfrak{g}) \rightarrow(0, \infty)$ satisfies $(36)$.

Theorem 5.2. Assume $(H 1-3)$ and let $\tau \in Z_{0}(\mathfrak{g})$ (i.e. $\mathrm{G}$ acts freely on $\left.\mu^{-1}(\tau)\right)$. The invariant $\Phi_{B, \Sigma}^{M, \mu-\tau}\left(\alpha ; \beta_{i} ; \gamma_{i}\right)$ is independent of the almost complex structure $J$ and the Hamiltonian perturbation $H$ used to define it. It depends only on the triple $(M, \omega, \mu)$, on the (co)homology classes $B, \alpha, \beta_{i}, \gamma_{i}$, and on the component of $\tau$ in $Z_{0}(\mathfrak{g})$.

Proof. The space $\mathcal{B}^{*} / \mathcal{G}$ depends on $M$, the G-action, $\Sigma$, and $P$. The invariant is defined by pairing an integral cohomology class on $\mathcal{B}^{*} / \mathcal{G}$, determined by $\alpha, \beta_{i}, \gamma_{i}$, with the homology class $\left[\mathcal{M}_{B, \Sigma}(\tau ; J, H)\right] \in H_{*}\left(\mathcal{B}^{*} / \mathcal{G} ; \mathbb{Z}\right)$. By Theorem 5.1, the latter is independent of $J$ and $H$ and depends only on the component of $\tau$ in $Z_{0}(\mathfrak{g})$. That it is also independent of the metric on $\Sigma$ follows by a similar cobordism argument.

Remark 5.3. The hypothesis that the Hamiltonian is small (compared to the volume of $\Sigma$ ) is quite restrictive. If we allow for more general (abstract) perturbations of the symplectic vortex equations, then the condition that all solutions of (3) are irreducible can be replaced by the weaker condition that the gauge group acts freely on the space of solutions of (3). In the case of linear torus actions this condition is satisfied for every Hamiltonian perturbation whenever $\mathrm{G}$ acts freely on $\mu^{-1}\left(\tau_{0}\right)$, where $\tau_{0} \in \mathfrak{g}$ is defined by (20) (see Remark 4.3).

\section{G-moduli problems and the Euler class.}

In this section we review the results of [7] about the Euler class of G-moduli problems. They play a crucial role in the definition of the rational invariants in the presence of finite isotropy.

Definition 6.1. Let $\mathrm{G}$ be a compact oriented Lie group. A G-moduli problem is a triple $(\mathcal{B}, \mathcal{E}, \mathcal{S})$ with the following properties. 
- $\mathcal{B}$ is a Hilbert manifold equipped with a smooth G-action.

- $\mathcal{E}$ is a Hilbert space bundle over $\mathcal{B}$, also equipped with a smooth G-action, such that $\mathrm{G}$ acts by isometries on the fibres of $\mathcal{E}$ and the projection $\mathcal{E} \rightarrow \mathcal{B}$ is G-equivariant.

- $\mathcal{S}: \mathcal{B} \rightarrow \mathcal{E}$ is a smooth G-equivariant Fredholm section of constant Fredholm index such that the determinant bundle $\operatorname{det}(\mathcal{S}) \rightarrow \mathcal{B}$ is oriented, $\mathrm{G}$ acts by orientation preserving isomorphisms on the determinant bundle, and the zero set

$$
\mathcal{M}:=\{x \in \mathcal{B} \mid \mathcal{S}(x)=0\}
$$

is compact.

A finite dimensional G-moduli problem $(B, E, S)$ is called oriented if $B$ and $E$ are oriented and $\mathrm{G}$ acts on $B$ and $E$ by orientation preserving diffeomorphisms. A G-moduli problem $(\mathcal{B}, \mathcal{E}, \mathcal{S})$ is called regular if the isotropy subgroup $\mathrm{G}_{x}:=\left\{g \in \mathrm{G} \mid g^{*} x=x\right\}$ is finite for every $x \in \mathcal{M}$.

G-moduli problems form a category as follows.

Definition 6.2. Let $(\mathcal{B}, \mathcal{E}, \mathcal{S}),\left(\mathcal{B}^{\prime}, \mathcal{E}^{\prime}, \mathcal{S}^{\prime}\right)$ be G-moduli problems. A morphism from $(\mathcal{B}, \mathcal{E}, \mathcal{S})$ to $\left(\mathcal{B}^{\prime}, \mathcal{E}^{\prime}, \mathcal{S}^{\prime}\right)$ is a pair $(\psi, \Psi)$ with the following properties. $\psi: \mathcal{B}_{0} \rightarrow \mathcal{B}^{\prime}$ is a smooth G-equivariant embedding of a neighbourhood $\mathcal{B}_{0} \subset \mathcal{B}$ of $\mathcal{M}$ into $\mathcal{B}^{\prime}, \Psi: \mathcal{E}_{0}:=\left.\mathcal{E}\right|_{\mathcal{B}_{0}} \rightarrow \mathcal{E}^{\prime}$ is a smooth injective bundle homomorphism and a lift of $\psi$, and the sections $\mathcal{S}$ and $\mathcal{S}^{\prime}$ satisfy

$$
\mathcal{S}^{\prime} \circ \psi=\Psi \circ \mathcal{S}, \quad \mathcal{M}^{\prime}=\psi(\mathcal{M})
$$

Moreover, the linear operators $d_{x} \psi: T_{x} \mathcal{B} \rightarrow T_{\psi(x)} \mathcal{B}^{\prime}$ and $\Psi_{x}: \mathcal{E}_{x} \rightarrow \mathcal{E}_{\psi(x)}^{\prime}$ induce isomorphisms

$$
d_{x} \psi: \operatorname{ker} \mathcal{D}_{x} \rightarrow \operatorname{ker} \mathcal{D}_{\psi(x)}^{\prime}, \quad \Psi_{x}: \operatorname{coker} \mathcal{D}_{x} \rightarrow \operatorname{coker} \mathcal{D}_{\psi(x)}^{\prime},
$$

for $x \in \mathcal{M}$, and the resulting isomorphism from $\operatorname{det}(\mathcal{D})$ to $\operatorname{det}\left(\mathcal{D}^{\prime}\right)$ is orientation preserving.

Let $(\mathcal{B}, \mathcal{E}, \mathcal{S})$ and $\left(\mathcal{B}^{\prime}, \mathcal{E}^{\prime}, \mathcal{S}^{\prime}\right)$ be G-moduli problems and suppose that there exists a morphism from $(\mathcal{B}, \mathcal{E}, \mathcal{S})$ to $\left(\mathcal{B}^{\prime}, \mathcal{E}^{\prime}, \mathcal{S}^{\prime}\right)$. Then the indices of $\mathcal{S}$ and $\mathcal{S}^{\prime}$ agree. Moreover, $(\mathcal{B}, \mathcal{E}, \mathcal{S})$ is regular if and only if $\left(\mathcal{B}^{\prime}, \mathcal{E}^{\prime}, \mathcal{S}^{\prime}\right)$ is regular. 
Definition 6.3. Two regular G-moduli problems $\left(\mathcal{B}, \mathcal{E}_{i}, \mathcal{S}_{i}\right), i=0,1$, (over the same base) are called homotopic if there exists a G-equivariant Hilbert space bundle $\mathcal{E} \rightarrow[0,1] \times \mathcal{B}$ and a G-equivariant smooth section $\mathcal{S}:[0,1] \times$ $\mathcal{B} \rightarrow \mathcal{E}$ such that $\mathcal{E}_{i}=\left.\mathcal{E}\right|_{\{i\} \times \mathcal{B}}$ and $\mathcal{S}_{i}=\left.\mathcal{S}\right|_{\{i\} \times \mathcal{B}}$ for $i=0$, 1, the triple $\left(\mathcal{B}, \mathcal{E}_{t}, \mathcal{S}_{t}\right)$, defined by $\mathcal{E}_{t}:=\left.\mathcal{E}\right|_{\{t\} \times \mathcal{B}}$ and $\mathcal{S}_{t}=\left.\mathcal{S}\right|_{\{t\} \times \mathcal{B}}$, is a regular G-moduli problem for every $t \in[0,1]$, and the set $\mathcal{M}:=\left\{(t, x) \in[0,1] \times \mathcal{B} \mid \mathcal{S}_{t}(x)=0\right\}$ is compact.

The following theorem in proved in [7]. It states the properties of the Euler class. We denote by $H_{\mathrm{G}}^{*}(\mathcal{B})$ the equivariant cohomology with real coefficients.

Theorem 6.4. There exists a functor, called the Euler class, which assigns to each compact oriented Lie group $\mathrm{G}$ and each regular $\mathrm{G}-$ moduli problem $(\mathcal{B}, \mathcal{E}, \mathcal{S})$ a homomorphism $\chi^{\mathcal{B}, \mathcal{E}, \mathcal{S}}: H_{\mathrm{G}}^{*}(\mathcal{B}) \rightarrow \mathbb{R}$ and satisfies the following.

(Functoriality) If $(\psi, \Psi)$ is a morphism from $(\mathcal{B}, \mathcal{E}, \mathcal{S})$ to $\left(\mathcal{B}^{\prime}, \mathcal{E}^{\prime}, \mathcal{S}^{\prime}\right)$ then $\chi^{\mathcal{B}, \mathcal{E}, \mathcal{S}}\left(\psi^{*} \alpha\right)=\chi^{\mathcal{B}^{\prime}, \mathcal{E}^{\prime}, \mathcal{S}^{\prime}}(\alpha)$ for every $\alpha \in H_{\mathrm{G}}^{*}\left(\mathcal{B}^{\prime}\right)$.

(Thom class) If $(B, E, S)$ is a finite dimensional oriented regular Gmoduli problem and $\tau \in \Omega_{\mathrm{G}}^{*}(E)$ is an equivariant Thom form supported in an open neighbourhood $U \subset E$ of the zero section such that $U \cap E_{x}$ is convex for every $x \in B, U \cap \pi^{-1}(K)$ has compact closure for every compact set $K \subset B$, and $S^{-1}(U)$ has compact closure, then

$$
\chi^{B, E, S}(\alpha)=\int_{B / G} \alpha \wedge S^{*} \tau
$$

for every closed form $\alpha \in \Omega_{\mathrm{G}}^{*}(B)$.

(Transversality) If $\mathcal{S}$ is transverse to the zero section then

$$
\chi^{\mathcal{B}, \mathcal{E}, \mathcal{S}}(\alpha)=\int_{\mathcal{M} / \mathrm{G}} \alpha
$$

for every $\alpha \in H_{\mathrm{G}}^{*}(B)$, where $\mathcal{M}:=\mathcal{S}^{-1}(0)$.

(Homotopy) If $\left(\mathcal{B}, \mathcal{E}_{0}, \mathcal{S}_{0}\right)$ and $\left(\mathcal{B}, \mathcal{E}_{1}, \mathcal{S}_{1}\right)$ are regular homotopic $\mathrm{G}$-moduli problems then $\chi^{\mathcal{B}, \mathcal{E}_{0}, \mathcal{S}_{0}}(\alpha)=\chi^{\mathcal{B}, \mathcal{E}_{1}, \mathcal{S}_{1}}(\alpha)$ for every $\alpha \in H_{\mathrm{G}}^{*}(\mathcal{B})$.

(Subgroup) If $(\mathcal{B}, \mathcal{E}, \mathcal{S})$ is a regular $\mathrm{G}$-moduli problem and $\mathrm{H} \subset \mathrm{G}$ is a normal subgroup acting freely on $\mathcal{B}$ then

$$
\chi^{\mathcal{B} / \mathrm{H}, \mathcal{E} / \mathrm{H}, \mathcal{S} / \mathrm{H}}(\alpha)=\chi^{\mathcal{B}, \mathcal{E}, \mathcal{S}}\left(\pi^{*} \alpha\right)
$$


for every $\alpha \in H_{\mathrm{G} / \mathrm{H}}^{*}(\mathcal{B} / \mathrm{H})$, where $\pi^{*}: H_{\mathrm{G} / \mathrm{H}}^{*}(\mathcal{B} / \mathrm{H}) \rightarrow H_{\mathrm{G}}^{*}(\mathcal{B})$ is the homomorphism induced by the projection $\pi: \mathcal{B} \rightarrow \mathcal{B} / \mathrm{H}$.

(Rationality) If $\alpha \in H_{\mathrm{G}}^{*}(\mathcal{B} ; \mathbb{Q})$ then $\chi^{\mathcal{B}, \mathcal{E}, \mathcal{S}}(\alpha) \in \mathbb{Q}$.

The Euler class is uniquely determined by the (Functoriality) and (Thom class) axioms.

\section{Rational invariants.}

Our next goal is to drop the hypothesis that $\mathrm{G}$ acts freely on $\mu^{-1}(\tau)$ and construct invariants for every central regular values $\tau \in Z(\mathfrak{g})$ of the moment map $\mu: M \rightarrow \mathfrak{g}$. In this case we must deal with the presence of finite isotropy subgroups. We assume as before that the hypotheses $(H 1-3)$ are satisfied.

\subsection{The setup.}

Let us fix the following data:

- an equivariant homology class $B \in H_{2}\left(M_{\mathrm{G}} ; \mathbb{Z}\right)$,

- a compact connected Riemann surface $\left(\Sigma, J_{\Sigma}, \mathrm{dvol}_{\Sigma}\right)$ and a principal Gbundle $\pi: P \rightarrow \Sigma$ whose characteristic class is the image of $B$ under the homomorphism $H_{2}\left(M_{\mathrm{G}} ; \mathbb{Z}\right) \rightarrow H_{2}(\mathrm{BG} ; \mathbb{Z})$,

- a point $p_{0} \in P$ and an integer $k \geq 2$.

We emphasize that the purpose of fixing the point $p_{0}$ is not in the definition of the evaluation map, but to obtain an action of the gauge group on the classifying space EG of G. Throughout we shall denote by $\mathcal{G}:=\mathcal{G}^{k+1,2}(P)$ the group of gauge transformations of $P$ of class $W^{k+1,2}$ and by

$$
\mathcal{G}_{0}:=\mathcal{G}_{0}^{k+1,2}(P):=\left\{g \in \mathcal{G}^{k+1,2}(P) \mid g\left(p_{0}\right)=\mathbb{1}\right\}
$$

the (normal) subgroup of based gauge transformation. When the need arises we shall think of the gauge group $\mathcal{G}$ as acting on EG by $g^{*} e:=g\left(p_{0}\right)^{-1} e$. So the subgroup $\mathcal{G}_{0}$ acts trivially on EG.

The above data give rise to a $\mathrm{G}$-moduli problem $(\mathcal{B}, \mathcal{E}, \mathcal{S})$ as follows. The Hilbert manifold $\mathcal{B}$ is the quotient

$$
\mathcal{B}:=\frac{W_{\mathrm{G}}^{k, 2}(P, M ; B) \times \mathcal{A}^{k, 2}(P)}{\mathcal{G}_{0}^{k+1,2}(P)},
$$


where $W_{\mathrm{G}}^{k, 2}(P, M ; B):=\left\{\left(u \in W_{\mathrm{G}}^{k, 2}(P, M) \mid[u]=B\right\}\right.$. The Hilbert manifold $\mathcal{B}$ carries a $\mathrm{G}$-action since the quotient group $\mathcal{G} / \mathcal{G}_{0}$ is isomorphic to $\mathrm{G}$. Consider the bundle $\mathcal{E} \rightarrow \mathcal{B}$ with fibres

$$
\mathcal{E}_{(u, A)}:=W^{k-1,2}\left(\Sigma, \Lambda_{J_{\Sigma}}^{0,1} T^{*} \Sigma \otimes_{J} u^{*} T M / \mathrm{G}\right) \oplus W^{k-1,2}\left(\Sigma, \mathfrak{g}_{P}\right) .
$$

The action of the gauge group identifies $\mathcal{E}_{(u, A)}$ with $\mathcal{E}_{\left(g^{-1} u, g^{*} A\right)}$ for every $g \in \mathcal{G}$. Thus $\mathcal{E}$ carries a G-action. More precisely, the fibre of $\mathcal{E}$ over a point $[u, A] \in \mathcal{B}$ is the union of the spaces $\mathcal{E}_{\left(g^{-1} u, g^{*} A\right)}$ over all $g \in \mathcal{G}_{0}$ and any two such spaces are identified by the action of the based gauge group. Then the quotient group $\mathrm{G} \cong \mathcal{G} / \mathcal{G}_{0}$ acts on both $\mathcal{E}$ and $\mathcal{B}$ and the projection is G-equivariant. For every Hamiltonian perturbation $H$ the left hand side of equation (3) defines a G-equivariant section $\mathcal{S}: \mathcal{B} \rightarrow \mathcal{E}$ given by

$$
\mathcal{S}([u, A]):=\left[\bar{\partial}_{J, H, A}(u), * F_{A}+\mu(u)-\tau\right] .
$$

Lemma 7.1. Assume $(H 1-3)$ and let $\tau \in Z(\mathfrak{g})$ be a regular value of $\mu$.

(i) The triple $(\mathcal{B}, \mathcal{E}, \mathcal{S})$ defined by $(39),(40)$, and (41) is a $\mathrm{G}$-moduli problem (see Section 6$)$ of index

$$
\operatorname{index}(\mathcal{S})=(n-\operatorname{dim} \mathrm{G}) \chi(\Sigma)+2\left\langle c_{1}^{\mathrm{G}}(T M), B\right\rangle=: 2 m
$$

(ii) There exists a constant $\delta>0$ such that the $\mathrm{G}$-moduli problem $(\mathcal{B}, \mathcal{E}, \mathcal{S})$ is regular whenever

$$
\langle[\omega-\mu+\tau], B\rangle+\left\|\Omega_{H}\right\| \leq \delta \operatorname{Vol}(\Sigma) .
$$

Proof. That $\mathcal{E}$ is a Hilbert space bundle over a Hilbert manifold is a consequence of the local slice theorem (for $W^{k, 2}$ connections). That $\mathcal{S}$ is a Fredholm section follows from Proposition 4.6 and so does the index formula. That the zero set of $\mathcal{S}$ is compact follows from Corollary 3.5. This proves (i). Assertion (ii) follows from Lemma 4.2.

We can now evaluate the Euler class $\chi^{\mathcal{B}, \mathcal{E}, \mathcal{S}}$, defined in Section 6 , on equivariant cohomology classes of $\mathcal{B}$. As in Section 5.2, such cohomology classes can be obtained by pulling back equivariant cohomology classes on $M$ with the evaluation map and equivariant cohomology classes on $\mathcal{A} / \mathcal{G}_{0}$ with the projection onto the space of connections. More precisely, abbreviate 
$\mathcal{A}:=\mathcal{A}^{k, 2}(P)$ and $\mathcal{G}=\mathcal{G}^{k+1,2}(P)$. Consider the action of the group $\mathcal{G} \times \mathrm{G}$ on the space

$$
\mathcal{X}:=\mathcal{A} \times P \times \mathrm{EG}
$$

by

$$
(g, h)^{*}(A, p, e):=\left(g^{*} A, p g(p)^{-1} h, g\left(p_{0}\right)^{-1} e\right) .
$$

Lemma 7.2. There exists a continuous function $\theta: \mathcal{X} \rightarrow$ EG which is $\mathcal{G}$-invariant and $\mathrm{G}$-equivariant. Thus

$$
\theta\left(g^{*} A, p g(p)^{-1} h, g\left(p_{0}\right)^{-1} e\right)=h^{-1} \theta(A, p, e)
$$

for $(A, p, e) \in \mathcal{X}, g \in \mathcal{G}$, and $h \in \mathrm{G}$. Any two such maps $\theta_{0}, \theta_{1}: \mathcal{X} \rightarrow \mathrm{EG}$ are homotopic through maps satisfying (43). Moreover, $\theta$ can be chosen such that

$$
\theta\left(A, p_{0}, e\right)=e
$$

Proof. The group $\mathcal{G} \times \mathrm{G}$ acts freely on $\mathcal{X}$. Hence the quotient $\mathcal{X} / \mathcal{G}$ is a principal G-bundle over $\mathcal{X} /(\mathcal{G} \times \mathrm{G})$. The classifying map of this bundle lifts to a G-equivariant map from $\mathcal{X} / \mathcal{G}$ to EG. The composition of this map with the projection $\mathcal{X} \rightarrow \mathcal{X} / \mathcal{G}$ is the required map $\theta$. The last two assertions follow from the fact that any two classifying maps are equivariantly homotopic.

Note that we can identify the space $\mathcal{B} \times{ }_{\mathrm{G}}$ EG with the quotient of the space $W_{\mathrm{G}}^{k, 2}(P, M ; B) \times \mathcal{A}^{k, 2}(P) \times \mathrm{EG}$ by $\mathcal{G}=\mathcal{G}^{k+1,2}(P)$, where the action of the gauge group is given by $g^{*}(u, A, e):=\left(g^{-1} u, g^{*} A, g\left(p_{0}\right)^{-1} e\right)$. Hence there is an evaluation map

$$
\mathrm{ev}_{\mathrm{G}}:\left(\mathcal{B} \times_{\mathrm{G}} \mathrm{EG}\right) \times \Sigma \rightarrow M \times_{\mathrm{G}} \mathrm{EG},
$$

defined by

$$
\operatorname{ev}_{\mathrm{G}}([u, A, e], \pi(p)):=[u(p), \theta(A, p, e)],
$$

and a projection

$$
\pi_{\mathcal{A}}: \mathcal{B} \times_{\mathrm{G}} \mathrm{EG} \rightarrow \mathcal{A}_{\mathcal{G}}:=\mathcal{A} \times_{\mathcal{G}} \mathrm{EG}
$$

defined by

$$
\pi_{\mathcal{A}}([u, A, e]):=[A, e] .
$$




\subsection{Definition of the invariants.}

Let

$$
\alpha \in H^{*}\left(\mathcal{A}_{\mathcal{G}}\right), \quad \beta_{1}, \ldots, \beta_{k} \in H^{*}\left(M_{\mathrm{G}}\right), \quad \gamma_{1}, \ldots, \gamma_{k} \in H_{*}(\Sigma)
$$

such that

$$
\operatorname{deg}(\alpha)+\sum_{i=1}^{k} \operatorname{deg}\left(\beta_{i}\right)-\sum_{i=1}^{k} \operatorname{deg}\left(\gamma_{i}\right)=2 m
$$

and define

$$
\begin{aligned}
& \Phi_{B, \Sigma}^{M, \mu-\tau}\left(\alpha ; \beta_{1}, \ldots, \beta_{k} ; \gamma_{1}, \ldots, \gamma_{k}\right) \\
& :=\chi^{\mathcal{B}, \mathcal{E}, \mathcal{S}}\left(\pi_{\mathcal{A}}^{*} \alpha \smile \mathrm{ev}_{\mathrm{G}}^{*} \beta_{1} / \gamma_{1} \smile \cdots \smile \mathrm{ev}_{\mathrm{G}}^{*} \beta_{k} / \gamma_{k}\right) .
\end{aligned}
$$

Here the map $H^{q}\left(\left(\mathcal{B} \times{ }_{\mathrm{G}} \mathrm{EG}\right) \times \Sigma\right) \times H_{i}(\Sigma) \rightarrow H^{q-i}\left(\mathcal{B} \times{ }_{\mathrm{G}} \mathrm{EG}\right):(\beta, \gamma) \mapsto \beta / \gamma$ denotes the slant product, the $\mathrm{G}$-moduli problem $(\mathcal{B}, \mathcal{E}, \mathcal{S})$ is defined by $(39)$, (40), and (41), where the Hamiltonian perturbation $H$ satisfies (42), and the Euler class $\chi^{\mathcal{B}, \mathcal{E}, \mathcal{S}}: H_{\mathrm{G}}^{*}(\mathcal{B}) \rightarrow \mathbb{R}$ is defined in Section 6 .

Theorem 7.3. Assume $(H 1-3)$ and let $\tau \in Z(\mathfrak{g})$ be a regular value of $\mu$. The invariant $\Phi_{B, \Sigma}^{M, \mu-\tau}\left(\alpha ; \beta_{i} ; \gamma_{i}\right)$ is independent of the almost complex structure $J$, the Hamiltonian perturbation $H$, the point $p_{0} \in P$, and the integer $k$ used to define it. It depends only on $(M, \omega, \mu)$, on the genus of $\Sigma$, on the component of $\tau$ in the (open) set of central regular values of $\mu$, and on the (co)homology classes $B, \alpha, \beta_{i}, \gamma_{i}$.

Proof. The independence of $k$ follows from the fact that a finite dimensional reduction for $k=2$ is also a finite dimensional reduction for every $k>2$, and that the classifying map $\theta^{k, 2}: \mathcal{X}^{k, 2} \rightarrow$ EG can be defined as the restriction of the classifying map $\theta^{2,2}$ to the subspace $\mathcal{X}^{k, 2} \subset \mathcal{X}^{2,2}$. The independence of $J, H, J_{\Sigma}, \mathrm{dvol}_{\Sigma}$, and $\tau$ follows from the (Homotopy) axiom for the Euler class.

We prove the independence of the basepoint $p_{0}$. Let $p_{1} \in P$ and suppose that $H=0$ and that $J$ is independent of the point $z \in \Sigma$. Choose a diffeomorphism $\phi: \Sigma \rightarrow \Sigma$ that is isotopic to the identity and a G-equivariant lift $\psi: P \rightarrow P$ such that $\psi\left(p_{1}\right)=p_{0}$. Then the G-moduli problem with $p_{0}, J_{\Sigma}$, and $\mathrm{dvol}_{\Sigma}$ replaced by $p_{1}=\psi^{-1}\left(p_{0}\right), \phi^{*} J_{\Sigma}$, and $\phi^{*} \mathrm{dvol}_{\Sigma}$, respectively, is diffeomorphic to the original one. The diffeomorphism is given by $[u, A] \mapsto\left[u \circ \psi, \psi^{*} A\right]$. Hence the invariants are the same. 
Remark 7.4. We emphasize again that the condition (42) on the Hamiltonian perturbation is quite restrictive and that much more general regularity criteria are available in the abelian case. For example, if $\mathrm{G}$ is abelian and acts linearly on $M=\mathbb{C}^{n}$ and the element $\tau_{0} \in \mathfrak{g}$, defined by (20), is a regular value of $\mu$ then, for every Hamiltonian perturbation and every almost complex structure, the gauge group acts on the space of solutions of (3) with finite isotropy (see Remarks 4.3). So in this case the smallness condition (42) on the Hamiltonian can be dropped. Such more general criteria can also be obtained in the nonabelian case.

Theorem 7.5. Assume $(H 1-3)$ and let $\tau \in Z(\mathfrak{g})$ such that $\mathrm{G}$ acts freely on $\mu^{-1}(\tau)$. Then the invariant $\Phi_{B, \Sigma}^{M, \mu-\tau}\left(\alpha ; \beta_{i} ; \gamma_{i}\right)$ defined in this section agrees with the one defined in Section 5.2.

Proof. By Theorem 5.1, there exists a Hamiltonian perturbation $H$ that satisfies (42) and is regular in the sense of Definition 4.9. For such a perturbation the section $\mathcal{S}: \mathcal{B} \rightarrow \mathcal{E}$, defined by (41), is transverse to the zero section. Hence the result follows from the (Transversality) axiom for the Euler class.

\subsection{Relations.}

There are two kinds of relations between the invariants defined in Section 7.2, namely those arising from relations between the slant product and the cup product and others arising from relations between certain universal bundles in gauge theory.

Proposition 7.6. Let $\iota: \Sigma \rightarrow \Sigma \times \Sigma$ denote the inclusion of the diagonal and suppose that $\beta, \beta^{\prime}, \beta^{\prime \prime} \in H^{*}\left(M_{\mathrm{G}} ; \mathbb{Z}\right)$ and $\gamma, \gamma_{i}^{\prime}, \gamma_{i}^{\prime \prime} \in H_{*}(\Sigma ; \mathbb{Z})$ satisfy

$$
\beta=\beta^{\prime} \smile \beta^{\prime \prime}, \quad \iota_{*} \gamma=\sum_{i=1}^{m} \gamma_{i}^{\prime} \otimes \gamma_{i}^{\prime \prime} .
$$

Then

$$
\begin{aligned}
& \Phi_{B, \Sigma}^{M, \mu-\tau}\left(\alpha ; \beta, \beta_{1}, \ldots, \beta_{k} ; \gamma, \gamma_{1}, \ldots, \gamma_{k}\right) \\
& =\sum_{i=1}^{m}(-1)^{\operatorname{deg}\left(\gamma_{i}^{\prime}\right) \operatorname{deg}\left(\beta^{\prime \prime} / \gamma_{i}^{\prime \prime}\right)} \Phi_{B, \Sigma}^{M, \mu-\tau}\left(\alpha ; \beta^{\prime}, \beta^{\prime \prime}, \beta_{1}, \ldots, \beta_{k} ; \gamma_{i}^{\prime}, \gamma_{i}^{\prime \prime}, \gamma_{1}, \ldots, \gamma_{k}\right) .
\end{aligned}
$$


In particular, if $\gamma_{i}=[\mathrm{pt}]$ for every $i$, then

$$
\Phi_{B, \Sigma}^{M, \mu-\tau}\left(\alpha ; \beta_{1}, \ldots, \beta_{k} ; \mathrm{pt}, \ldots, \mathrm{pt}\right)=\Phi_{B, \Sigma}^{M, \mu-\tau}\left(\alpha ; \beta_{1} \smile \cdots \smile \beta_{k} ; \mathrm{pt}\right) .
$$

Proof. This follows from the formula

$$
\int_{\gamma} \alpha^{\prime} \smile \alpha^{\prime \prime}=\sum_{i=1}^{m} \int_{\gamma_{i}^{\prime}} \alpha^{\prime} \int_{\gamma_{i}^{\prime \prime}} \alpha^{\prime \prime}
$$

for $\alpha^{\prime}, \alpha^{\prime \prime} \in H^{*}(\Sigma ; \mathbb{Z})$.

Let us denote by

$$
\theta_{\mathcal{A}}: \mathcal{A}_{\mathcal{G}} \times \Sigma \rightarrow \mathrm{BG}
$$

the map

$$
\theta_{\mathcal{A}}([A, e], \pi(p)):=[\theta(A, p, e)],
$$

where $\theta$ is as in Lemma 7.2. This is a classifying map for the bundle

$$
\mathcal{P}_{\mathcal{A}}:=(\mathcal{A} \times P \times \mathrm{EG}) / \mathcal{G} \rightarrow \mathcal{A}_{\mathcal{G}} \times \Sigma,
$$

where the gauge group $\mathcal{G}$ acts by $g^{*}(A, p, e):=\left(g^{*} A, p g(p)^{-1}, g\left(p_{0}\right)^{-1} e\right)$. Recall that $\rho_{M}: M_{\mathrm{G}} \rightarrow \mathrm{BG}$ denotes the projection.

Proposition 7.7. For every $c \in H^{*}(\mathrm{BG} ; \mathbb{Z})$ and every $\gamma \in H_{*}(\Sigma ; \mathbb{Z})$,

$$
\begin{aligned}
& \Phi_{B, \Sigma}^{M, \mu-\tau}\left(\alpha ; \rho_{M}^{*} c, \beta_{1}, \ldots, \beta_{k} ; \gamma, \gamma_{1}, \ldots, \gamma_{k}\right) \\
& =\Phi_{B, \Sigma}^{M, \mu-\tau}\left(\alpha \smile\left(\theta_{\mathcal{A}}^{*} c / \gamma\right) ; \beta_{1}, \ldots, \beta_{k} ; \gamma_{1}, \ldots, \gamma_{k}\right) .
\end{aligned}
$$

Proof. By definition of the maps, there is a commuting diagram

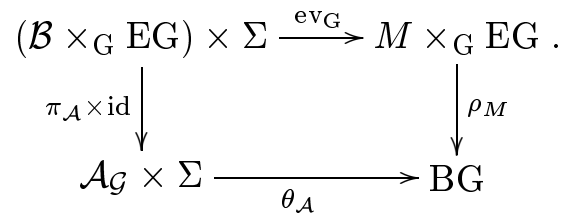

Hence, for every class $c \in H^{*}(\mathrm{BG} ; \mathbb{Z})$ and every $\gamma \in H_{*}(\Sigma ; \mathbb{Z})$, we have

$$
\operatorname{ev}_{G}^{*} \rho_{M}^{*} c / \gamma=\left(\left(\pi_{\mathcal{A}} \times \mathrm{id}\right)^{*} \theta_{\mathcal{A}}^{*} c\right) / \gamma=\pi_{\mathcal{A}}^{*}\left(\theta_{\mathcal{A}}^{*} c / \gamma\right)
$$

This proves the proposition. 
Let us now consider the abelian case $\mathrm{G}=T$. Then the constant gauge transformations act trivially on $\mathcal{A}$. Hence there is a principal bundle

$$
\mathcal{P}_{\mathcal{A} / \mathcal{G}}:=(\mathcal{A} \times P) / \mathcal{G}_{0} \rightarrow \mathcal{A} / \mathcal{G} \times \Sigma,
$$

where $\mathcal{G}_{0}$ is the based gauge group. Let us denote by

$$
\theta_{\mathcal{A} / \mathcal{G}}: \mathcal{A} / \mathcal{G} \times \Sigma \rightarrow \text { BG. }
$$

It lifts to a map $\theta_{0}: \mathcal{A} \times P \rightarrow$ EG that satisfies

$$
\theta_{0}\left(g^{*} A, p g(p)^{-1}\right)=g\left(p_{0}\right)^{-1} \theta_{0}(A, p) .
$$

Consider the homomorphism $\mu_{\mathcal{A} / \mathcal{G}}: H^{q}(\mathrm{BG} ; \mathbb{Z}) \times H_{i}(\Sigma ; \mathbb{Z}) \rightarrow H^{q-i}(\mathcal{A} / \mathcal{G} ; \mathbb{Z})$ defined by

$$
\mu_{\mathcal{A} / \mathcal{G}}(c, \gamma):=\theta_{\mathcal{A} / \mathcal{G}^{c}} / \gamma \in H^{*}(\mathcal{A} / \mathcal{G} ; \mathbb{Z})
$$

Let $\pi_{\mathcal{A} / \mathcal{G}}: \mathcal{A}_{\mathcal{G}} \rightarrow \mathcal{A} / \mathcal{G}$ denote the obvious projection.

Proposition 7.8. Assume the abelian case $\mathrm{G}=T$. Then, for every $c \in$ $H^{*}(\mathrm{BG} ; \mathbb{Z})$ and every $\gamma \in H_{*}(\Sigma ; \mathbb{Z})$ such that

$$
\operatorname{deg}(\gamma)>0
$$

we have

$$
\begin{aligned}
& \Phi_{B, \Sigma}^{M, \mu-\tau}\left(\alpha ; \rho_{M}^{*} c, \beta_{1}, \ldots, \beta_{k} ; \gamma, \gamma_{1}, \ldots, \gamma_{k}\right) \\
& =\Phi_{B, \Sigma}^{M, \mu-\tau}\left(\alpha \smile \pi_{\mathcal{A} / \mathcal{G}}^{*} \mu_{\mathcal{A} / \mathcal{G}}(c, \gamma) ; \beta_{1}, \ldots, \beta_{k} ; \gamma_{1}, \ldots, \gamma_{k}\right) .
\end{aligned}
$$

Proof. In the abelian case the projection

$$
\mathcal{A}_{\mathcal{G}} \rightarrow \mathcal{A} / \mathcal{G} \times \mathrm{BG}
$$

is a homeomorphism. We define the tensor product of two principal $T$ bundles $\pi_{1}: P_{1} \rightarrow X$ and $\pi_{2}: P_{2} \rightarrow X$ as the quotient

$$
P_{1} \otimes P_{2}:=\left\{\left(p_{1}, p_{2}\right) \in P_{1} \times P_{2} \mid \pi_{1}\left(p_{1}\right)=\pi_{2}\left(p_{2}\right)\right\} / T
$$

by the diagonal action. With this notation

$$
\mathcal{P}_{\mathcal{A}} \cong\left(\mathcal{P}_{A / \mathcal{G}} \times \mathrm{BG}\right) \otimes(\mathcal{A} / \mathcal{G} \times \Sigma \times \mathrm{EG}) \longrightarrow \mathcal{A} / \mathcal{G} \times \Sigma \times \mathrm{BG}
$$

An explicit bundle isomorphism is $[A, p, e] \mapsto\left[\left([A, p]_{0},[e]\right),([A],[p], e)\right]$, where $[A, p]_{0} \in \mathcal{P}_{\mathcal{A} / \mathcal{G}}=(\mathcal{A} \times P) / \mathcal{G}_{0}$ denotes the equivalence class under 
the action of $\mathcal{G}_{0}$, and $[A]$, respectively $[p]$ and $[e]$, denote the equivalence classes under the action of $\mathcal{G}$, respectively G. Hence

$$
\operatorname{deg}(\gamma)>0 \quad \Longrightarrow \quad \theta_{\mathcal{A}}^{*} c / \gamma=\pi_{\mathcal{A} / \mathcal{G}}^{*}\left(\theta_{\mathcal{A} / \mathcal{G}}^{*} c / \gamma\right)=\pi_{\mathcal{A} / \mathcal{G}}^{*} \mu_{\mathcal{A} / \mathcal{G}}(c, \gamma)
$$

and hence the result follows from Proposition 7.7.

The classes $\mu_{A / \mathcal{G}}(c, \gamma)$ are easy to compute and they generate the cohomology of $\mathcal{A} / \mathcal{G}=\mathcal{A} / \mathcal{G}_{0}$. Let $\Lambda:=\exp ^{-1}(\mathbb{1}) \subset \mathfrak{t}$ and denote by $\mathrm{W} \subset \mathfrak{t}$ the dual lattice (of elements whose periods on $\Lambda$ are integer multiples of $2 \pi$ ). Then every $\mathrm{w} \in \mathrm{W}$ determines a homomorphism

$$
\rho_{\mathrm{w}}: T \rightarrow S^{1},
$$

given by $\rho_{\mathrm{w}}(\exp (\tau)):=\exp (i\langle\mathrm{w}, \tau\rangle)$ and hence complex line bundles

$$
\mathcal{L}^{\mathrm{w}}:=\mathrm{EG} \times \rho_{\mathrm{w}} \mathbb{C} \rightarrow \mathrm{BG}, \quad L^{\mathrm{w}}:=P \times \rho_{\mathrm{w}} \mathbb{C} \rightarrow \Sigma .
$$

The first Chern class of $\mathcal{L}^{\mathrm{w}}$ will be denoted by

$$
c_{\mathrm{w}}:=c_{1}\left(\mathcal{L}^{\mathrm{w}}\right) \in H^{2}(\mathrm{BG} ; \mathbb{Z}) .
$$

We describe the map $\gamma \mapsto \mu_{\mathcal{A} / \mathcal{G}}\left(c_{\mathrm{w}}, \gamma\right)$ explicitly. Every $\mathrm{w} \in \mathrm{W}$ and every loop $\gamma: S^{1} \rightarrow \Sigma$ determine a real valued 1 -form on $\mathcal{A}$ given by

$$
T_{A} \mathcal{A}=\Omega^{1}(\Sigma, \mathfrak{t}) \rightarrow \mathbb{R}: \alpha \mapsto-\frac{1}{2 \pi} \int_{\gamma}\langle\mathrm{w}, \alpha\rangle
$$

This 1 -form is closed and $\mathcal{G}$-invariant, so it descends to a closed 1 -form $\tilde{\mu}_{\mathrm{w}}(\gamma) \in \Omega^{1}(\mathcal{A} / \mathcal{G})$. Similarly, every 2 -chain $\sigma$ on $\Sigma$ induces a function $\tilde{\mu}(\sigma)$ on $\mathcal{A} / \mathcal{G}$ defined by

$$
\tilde{\mu}_{\mathrm{w}}(\sigma)([A]):=-\frac{1}{2 \pi} \int_{\sigma}\left\langle\mathrm{w}, F_{A}\right\rangle
$$

By Stokes' Theorem, $d \tilde{\mu}_{\mathrm{w}}(\sigma)=\tilde{\mu}_{\mathrm{w}}(\partial \sigma)$ and hence there are induced homomorphisms $\tilde{\mu}_{\mathrm{w}}: H_{1}(\Sigma ; \mathbb{Z}) \rightarrow H^{1}(\mathcal{A} / \mathcal{G} ; \mathbb{Z})$ and $\tilde{\mu}_{\mathrm{w}}: H_{2}(\Sigma ; \mathbb{Z}) \rightarrow H^{0}(\mathcal{A} / \mathcal{G} ; \mathbb{Z})$. The following lemma asserts that $\tilde{\mu}_{\mathrm{w}}(\gamma)=\mu_{\mathcal{A} / \mathcal{G}}\left(c_{\mathrm{w}}, \gamma\right)$.

Lemma 7.9. For every $\mathrm{w} \in \mathrm{W}$ the following holds.

(i) $\mu_{\mathcal{A} / \mathcal{G}}\left(c_{\mathrm{w}},[p t]\right)=0$ and $\mu_{\mathcal{A} / \mathcal{G}}\left(c_{\mathrm{w}},[\Sigma]\right)=\left\langle c_{1}\left(L^{\mathrm{w}}\right),[\Sigma]\right\rangle \in H^{0}(\mathcal{A} / \mathcal{G} ; \mathbb{Z})$. 
(ii) For $\gamma \in H_{1}(\Sigma ; \mathbb{Z})$ the class $\mu_{\mathcal{A} / \mathcal{G}}\left(c_{\mathrm{w}}, \gamma\right) \in H^{1}(\mathcal{A} / \mathcal{G} ; \mathbb{Z})$ is represented by the closed 1 -form on $\mathcal{A} / \mathcal{G}$ induced by (44). The Poincaré dual of $\mu_{\mathcal{A} / \mathcal{G}}\left(c_{\mathrm{w}}, \gamma\right)$ is represented by the cycle

$$
C_{\mathcal{A} / \mathcal{G}}(\mathrm{w}, \gamma):=\left\{[A] \in \mathcal{A} / \mathcal{G} \mid \int_{\gamma}\left\langle\mathrm{w}, A-A_{0}\right\rangle=0\right\}
$$

with the orientation determined by $-\mathrm{w}$.

(iii) The map

$$
\mathrm{W} \otimes H_{1}(\Sigma ; \mathbb{Z}) \rightarrow H^{1}(\mathcal{A} / \mathcal{G} ; \mathbb{Z}):(\mathrm{w}, \gamma) \mapsto \mu_{\mathcal{A} / \mathcal{G}}\left(c_{\mathrm{w}}, \gamma\right)
$$

induces an isomorphism from the exterior algebra on the free $\mathbb{Z}$-module $\mathrm{W} \otimes H_{1}(\Sigma ; \mathbb{Z})$ to $H^{*}(\mathcal{A} / \mathcal{G} ; \mathbb{Z})$.

Proof. The bundle $\mathcal{P}_{\mathcal{A} / \mathcal{G}}=(\mathcal{A} \times P) / \mathcal{G}_{0} \rightarrow \mathcal{A} / \mathcal{G} \times \Sigma$ carries a universal connection induced by the $\mathcal{G}_{0}$-invariant 1 -form $\mathbb{A} \in \Omega^{1}(\mathcal{A} \times P, \mathfrak{t})$,

$$
\mathbb{A}_{(A, p)}(\alpha, v)=A_{p}(v)+\left(d^{*} d\right)^{-1} d^{*} \alpha(p) .
$$

Here $d^{*} d$ denotes the isomorphism

$$
d^{*} d: \Omega^{0}(\Sigma, \mathfrak{t}) \rightarrow \operatorname{im}\left(d^{*}: \Omega^{1}(\Sigma, \mathfrak{t}) \rightarrow \Omega^{0}(\Sigma, \mathfrak{t})\right)
$$

The curvature $F_{\mathbb{A}}$ of $\mathbb{A}$ is given by

$$
\left(F_{\mathbb{A}}\right)_{([A], z)}((\alpha, v),(\beta, w))=F_{A}(v, w)+\alpha_{z}(w)-\beta_{z}(v)
$$

for $v, w \in T_{z} \Sigma$ and $\alpha, \beta \in \Omega^{1}(\Sigma, i \mathbb{R})$. Let $[\gamma] \in H_{1}(\Sigma ; \mathbb{Z})$ and $[A] \in$ $H_{1}(\mathcal{A} / \mathcal{G} ; \mathbb{Z})$ be represented by loops $\gamma: \mathbb{R} / \mathbb{Z} \rightarrow \Sigma$ and $A: \mathbb{R} \rightarrow \mathcal{A}$ such that $A(t+1)=g^{*} A(t)$ for some $g \in \mathcal{G}$. Since the closed 2 -form $-\left\langle\mathrm{w}, F_{\mathbb{A}}\right\rangle / 2 \pi$ represents the cohomology class $c_{1}\left(\theta_{\mathcal{A} / \mathcal{G}}^{*} \mathcal{L}^{\mathrm{w}}\right)=\theta_{\mathcal{A} / \mathcal{G}}^{*} c_{\mathrm{w}}$, we have

$$
\begin{aligned}
\left\langle\mu_{\mathcal{A} / \mathcal{G}}\left(c_{\mathrm{w}},[\gamma]\right),[A]\right\rangle & =-\frac{1}{2 \pi} \int_{0}^{1} \int_{0}^{1}\left\langle\mathrm{w}, F_{\mathbb{A}}(\dot{A}(s), \dot{\gamma}(t))\right\rangle d s d t \\
& =-\int_{0}^{1}\left(\frac{1}{2 \pi} \int_{\gamma}\langle\mathrm{w}, \dot{A}(s)\rangle\right) d s \\
& =\left\langle\tilde{\mu}_{\mathrm{w}}([\gamma]),[A]\right\rangle .
\end{aligned}
$$


This proves (ii). Assertion (i) is proved similarly. To prove (iii) let $m:=$ $\operatorname{dim} T$ and pick integer bases $\mathrm{w}_{1}, \ldots, \mathrm{w}_{m}$ of $\mathrm{W}$ and $\gamma_{1}, \ldots, \gamma_{2 g}$ of $H_{1}(\Sigma ; \mathbb{Z})$. Now let $\alpha_{i j} \in \Omega^{1}(\Sigma, \mathfrak{t})$ be a harmonic 1-form such that

$$
-\frac{1}{2 \pi} \int_{\gamma_{i}}\left\langle\mathrm{w}_{j}, \alpha_{i^{\prime} j^{\prime}}\right\rangle=\delta_{i i^{\prime}} \delta_{j j^{\prime}}
$$

for $i, i^{\prime}=1, \ldots, 2 g$ and $j, j^{\prime}=1, \ldots, m$. Then the map

$$
\mathbb{T}^{2 g m}=\mathbb{R}^{2 g m} / \mathbb{Z}^{2 g m} \rightarrow \mathcal{A} / \mathcal{G}:[t] \mapsto A_{0}+\sum_{i, j} t_{i j} \alpha_{i j}
$$

is a homotopy equivalence and identifies the cohomology class of the 1-form $d t_{i j}$ with $\mu_{\mathcal{A} / \mathcal{G}}\left(c_{\mathrm{w}_{j}}, \gamma_{i}\right)$.

\section{Relative periodic orbits.}

Let $\mathrm{G}$ be a compact Lie group and $(M, \omega, \mu)$ be a symplectic manifold with a Hamiltonian G-action. Let $\mathbb{R} \times M \rightarrow \mathbb{R}:(t, x) \mapsto H_{t}(x)=H_{t+1}(x)$ be a G-invariant Hamiltonian. A relative periodic orbit is a pair $\left(x_{0}, g_{0}\right)$, where $g_{0} \in \mathrm{G}$ and $x_{0}: \mathbb{R} \rightarrow M$ is a smooth function such that

$$
\dot{x}_{0}(t)+X_{H_{t}}\left(x_{0}(t)\right)=0, \quad x_{0}(t+1)=g_{0} x_{0}(t) .
$$

It follows from the G-invariance of $H_{t}$ that the function $t \mapsto \mu\left(x_{0}(t)\right)$ is constant for every relative periodic orbit $\left(x_{0}, g_{0}\right)$. The group $\mathrm{G}$ acts on the space of relative periodic orbits by

$$
g^{*}\left(x_{0}, g_{0}\right):=\left(g^{-1} x_{0}, g^{-1} g_{0} g\right) .
$$

If $g_{0}$ belongs to the identity component of $\mathrm{G}$ then there exists a smooth function $g: \mathbb{R} \rightarrow \mathrm{G}$ such that

$$
g(t+1)=g_{0} g(t)
$$

Define $x: \mathbb{R} \rightarrow M$ and $\xi: \mathbb{R} \rightarrow \mathfrak{g}$ by

$$
x(t):=g(t)^{-1} x_{0}(t), \quad \xi(t):=g(t)^{-1} \dot{g}(t) .
$$

Then

(47) $\dot{x}(t)+X_{\xi(t)}(x(t))+X_{H_{t}}(x(t))=0, \quad x(t+1)=x(t), \quad \xi(t+1)=\xi(t)$.

A solution $(x, \xi)$ of $(47)$ is called contractible if the loop $x: \mathbb{R} / \mathbb{Z} \rightarrow M$ is contractible. A solution $\left(x_{0}, g_{0}\right)$ of $(45)$ is called contractible if there exists a smooth path $g: \mathbb{R} \rightarrow$ G satisfying (46) such that the loop $g^{-1} x_{0}: \mathbb{R} / \mathbb{Z} \rightarrow$ $M$ is contractible. 
Remark 8.1. The loop group $L \mathrm{G}:=C^{\infty}(\mathbb{R} / \mathbb{Z}, \mathrm{G})$ acts on the space of solutions of (47) by

$$
g^{*}(x, \xi):=\left(g^{-1} x, g^{-1} \dot{g}+g^{-1} \xi g\right) .
$$

If $M$ is compact then this action preserves the space of contractible loops. The proof uses Floer homology (see for example [24, Chapter 10]).

Remark 8.2. If $(x, \xi)$ is a solution of $(47)$ then

$$
\frac{d}{d t} \mu(x(t))+[\xi(t), \mu(x(t))]=0 .
$$

In particular, if $\mu(x(t)) \in Z(\mathfrak{g})$ for some $t \in \mathbb{R}$ then the function $t \mapsto \mu(x(t))$ is constant.

Theorem 8.3. Assume $(H 1-3)$ and suppose that $\tau \in Z(\mathfrak{g})$ is a regular value of $\mu$ such that $\mu^{-1}(\tau) \neq \emptyset$. Then, for every time dependent $\mathrm{G}$-invariant Hamiltonian $H_{t}=H_{t+1}: M \rightarrow \mathbb{R}$, there exists a contractible relative periodic orbit in $\mu^{-1}(\tau)$.

Corollary 8.4 (Gromov). Let $(M, \omega)$ be a compact symplectic manifold such that $\left\langle[\omega], \pi_{2}(M)\right\rangle=0$. Then every time-dependent 1-periodic Hamiltonian system on $M$ has a contractible periodic orbit.

Proof. Theorem 8.3 with $\mathrm{G}=\{\mathbb{1}\}$.

Corollary 8.5. Assume $(H 1-3)$ and suppose that $\mathrm{G}$ is abelian. Then, for every time dependent G-invariant Hamiltonian $H_{t}:=H_{t+1}: M \rightarrow \mathbb{R}$ and every $\tau \in \mathfrak{g}$ such that $\mu^{-1}(\tau) \neq \emptyset$, there exists a contractible relative periodic orbit in $\mu^{-1}(\tau)$.

Proof. We may assume without loss of generality that $\mathrm{G}=T$ is a torus and $M$ is connected. Then there exists a subgroup $\mathrm{H} \subset \mathrm{G}$, called the principal orbit type, such that $\mathrm{H}=\mathrm{G}_{x}$ for every $x$ in an open dense subset of $M$ (see Audin [1]). Since $\mathrm{H} \subset \mathrm{G}_{x}$ for every $x$ it follows that $\langle d \mu(x) v, \eta\rangle=0$ for all $v \in T_{x} M$ and all $\eta \in \mathfrak{h}:=\operatorname{Lie}(\mathrm{H})$. Since $M$ is connected this shows that the image of $\mu$ is contained in an affine subspace $\mathfrak{g}_{0} \subset \mathfrak{g}$ parallel to $\mathfrak{h}^{\perp}$. The assertion about the principal orbit type now shows that $\mu(M)$ is equal to the closure of its interior relative to $\mathfrak{g}_{0}$. Hence, for every $\tau \in \mu(M)$, there 
exists a sequence $\tau_{\nu} \in \mu(M)$ converging to $\tau$ such that $\tau_{\nu}$ is a regular value of the composition $\mu_{0}: M \rightarrow \mathfrak{g}_{0}$ of the moment map with the projection onto $\mathfrak{g}_{0}$. Now apply Theorem 8.3 to the action of $\mathrm{G} / \mathrm{H}$ on $M$ to obtain, for every $\nu$, a contractible relative periodic orbit $\left(x_{\nu}, g_{\nu}\right)$ in $\mu^{-1}\left(\tau_{\nu}\right)$. Every such sequence has a convergent subsequence.

Example 8.6. Consider a Hamiltonian action of $\mathrm{U}(2)$ on $(M, \omega)$ which factors through the determinant $\mathrm{U}(2) \rightarrow S^{1}$. Then the moment map has no central regular values.

Conjecture 8.7. Assume $(H 1)$. Then, for every time dependent G-invariant Hamiltonian $H_{t}:=H_{t+1}: M \rightarrow \mathbb{R}$ and every $\tau \in \mathfrak{g}$ such that $\mu^{-1}(\tau) \neq \emptyset$, there exists a contractible relative periodic orbit in $\mu^{-1}(\tau)$.

Example 8.8. Hypothesis $(H 1)$ cannot be removed in Conjecture 8.7. For example, consider the case $\mathrm{G}=\{\mathbb{1}\}$ and $M=\mathbb{T}^{2} \times \mathbb{R}^{2}$ with the Hamiltonian function $H(x, y)=a_{1} y_{1}+a_{2} y_{2}$, where $a_{1}$ and $a_{2}$ are rationally independent. Then there are no (relative) periodic orbits and the moment map is not proper.

Remark 8.9. It should be possible to extend the techniques developed in this paper to the case where (H3) is not satisfied, however, the moduli spaces will then no longer be compact. Such an extension should give rise to a proof of Conjecture 8.7 under hypotheses $(H 1)$ and $(H 2)$.

Remark 8.10. The proof of Theorem 8.3 shows that the result continues to hold if hypothesis $(H 3)$ is replaced by the condition

$$
\int_{0}^{1}\left(\max H_{t}-\min H_{t}\right) d t \leq \frac{1}{2} \min _{\substack{\text { const } \neq v: S^{2} \rightarrow M \\ \bar{\delta}_{J}(v)=0}} \int_{S^{2}} v^{*} \omega .
$$

Proof of Theorem 8.3. The proof is the analogue of Gromov's argument, with pseudoholomorphic curves replaced by the solutions of the perturbed symplectic vortex equations.

Let $P:=S^{2} \times \mathrm{G}$ be the trivial bundle and $B:=0 \in H_{2}\left(M \times_{\mathrm{G}} \mathrm{EG} ; \mathbb{Z}\right)$. We prove that

$$
\Phi_{0, S^{2}}^{M, \mu-\tau}(\alpha)=\int_{\mu^{-1}(\tau) / \mathrm{G}} \alpha
$$


for every $\alpha \in \Omega^{\operatorname{dim} M-2 \operatorname{dim} G}\left(M \times_{\mathrm{G}} \mathrm{EG}\right)$. To see this note that, by Lemma 2.3, every solution $(u, A) \in C^{\infty}\left(S^{2}, M\right) \times \Omega^{1}\left(S^{2}, \mathfrak{g}\right)$ of the unperturbed equation (1) over $S^{2}$ is horizontal. Since every flat G-connection on the trivial bundle over $S^{2}$ is gauge equivalent to the zero connection, it follows that every solution of (1) is gauge equivalent to a solution of the form

$$
u(z) \equiv x, \quad A=0 .
$$

For any such solution and any almost complex structure $J \in \mathcal{J}_{\mathrm{G}}(M, \omega)$ it follows from Remark 4.5 (with $H=0$ ) that the Cauchy-Riemann operator $D_{u, A}: C^{\infty}\left(S^{2}, u^{*} T M\right) \rightarrow \Omega^{0,1}\left(S^{2}, u^{*} T M\right)$ is complex linear. Moreover, the bundle $u^{*} T M \rightarrow S^{2}$ is a direct sum of complex line bundles of degree zero. Hence it follows from the Riemann-Roch theorem that $D_{u, A}$ is surjective. Combining these observations with Proposition 4.7 we find that the operator $\mathcal{D}_{u, A}$, defined by (23), is surjective. Now consider the setup of Section 7 and let $(\mathcal{B}, \mathcal{E}, \mathcal{S})$ be the G-moduli problem defined by $(39),(40)$, and (41). Let $(B, E, S)$ be the finite dimensional G-moduli problem defined by

$$
B:=E:=\mu^{-1}(\tau), \quad S \equiv 0 .
$$

Since $\mathcal{D}_{u, A}$ is surjective for every $[u, A] \in \mathcal{S}^{-1}(0)$, the obvious inclusions $B \hookrightarrow \mathcal{B}$ and $E \hookrightarrow \mathcal{E}$ define a morphism from $(B, E, S)$ to $(\mathcal{B}, \mathcal{E}, \mathcal{S})$ in the sense of Definition 6.2. Hence (48) follows from the (Functoriality) and (Transversality) axioms of the Euler class.

By Kirwan's theorem [19], the homomorphism

$$
H_{\mathrm{G}}^{*}(M) \rightarrow H_{\mathrm{G}}^{*}\left(\mu^{-1}(\tau)\right)
$$

is surjective for every $\tau \in Z(\mathrm{G})$. Since $\mu^{-1}(\tau)$ is nonempty and $\mathrm{G}$ acts with finite isotropy, there exists a G-invariant horizontal volume form on $\mu^{-1}(\tau)$. This implies that there exists a G-closed equivariant differential form $\alpha \in \Omega_{\mathrm{G}}^{\operatorname{dim} M-2 \operatorname{dim} \mathrm{G}}(M)$ such that

$$
\int_{\mu^{-1}(\tau) / G} \alpha \neq 0
$$

Hence, by Lemma 7.1 and Theorem 7.3, there exists a constant $\delta>0$ such that, for every metric on $S^{2}$, every $J \in \mathcal{J}_{\mathrm{G}}(M, \omega)$, and every compactly supported Hamiltonian perturbation $\widehat{H} \in \Omega^{1}\left(S^{2}, C_{\mathrm{G}}^{\infty}(M)\right)$,

$$
\left\|\Omega_{\widehat{H}}\right\| \leq \delta \operatorname{Vol}\left(S^{2}\right) \quad \Longrightarrow \quad \mathcal{M}_{0, S^{2}}(\tau ; J, \widehat{H}) \neq \emptyset
$$


For $T>0$ choose a metric on $S^{2}=\mathbb{C} \cup\{\infty\}$ such that the map $[-T, T] \times$ $\mathbb{R} / \mathbb{Z} \rightarrow \mathbb{C}:(s, t) \mapsto e^{2 \pi(s+i t)}$ is an isometric embedding. Let $\rho_{T}:[-T, T] \rightarrow$ $[0,1]$ be a smooth cutoff function sucht that $\pm \dot{\rho}_{T}(s) \geq 0$ for $\pm s \geq 0$ and $\rho_{T}(s)=1$ for $|s| \leq T-1$. Fix a compactly supported 1-periodic G-invariant Hamiltonian function $\mathbb{R} / \mathbb{Z} \times M \rightarrow \mathbb{R}:(t, x) \mapsto H_{t}(x)$. On the cylinder $[-T, T] \times \mathbb{R} / \mathbb{Z}$ consider the Hamiltonian perturbation $\widehat{H}_{T}:=\rho_{T}(s) H_{t}(x) d t$ and extend it by zero to all of $S^{2}$. The Hofer norm of the curvature of $\widehat{H}_{T}$ is given by

$$
\left\|\Omega_{\widehat{H}_{T}}\right\|=2\|H\|, \quad\|H\|:=\int_{0}^{1}\left(\max H_{t}-\min H_{t}\right) d t .
$$

Hence it follows from (49), that

$$
\mathcal{M}_{0, S^{2}}\left(\tau ; J, \widehat{H}_{T}\right) \neq \emptyset
$$

for $T$ sufficiently large. This implies that for $T \geq T_{0}$ there exist functions $u=u_{T}:[-T, T] \times \mathbb{R} / \mathbb{Z} \rightarrow M$ and $\Phi=\Phi_{T}, \Psi=\Psi_{T}:[-T, T] \times \mathbb{R} / \mathbb{Z} \rightarrow \mathfrak{g}$ such that

$$
\begin{array}{r}
\partial_{s} u+X_{\Phi}(u)+J\left(\partial_{t} u+X_{\Psi}(u)+\rho_{T}(s) X_{H_{t}}(u)\right)=0 \\
\partial_{s} \Psi-\partial_{t} \Phi+[\Phi, \Psi]+\mu(u)-\tau=0
\end{array}
$$

and

$$
\int_{0}^{1} \int_{-T+1}^{T-1}\left(\left|\partial_{t} u+L_{u} \Psi+X_{H_{t}}(u)\right|^{2}+|\mu(u)-\tau|^{2}\right) d s d t \leq 2\|H\| .
$$

The inequality (51) follows from the energy identity in Proposition 2.2. Choose $s_{T} \in[-T+1, T-1]$ such that

$$
\int_{0}^{1}\left(\left|\partial_{t} u+L_{u} \Psi+X_{H_{t}}(u)\right|^{2}+|\mu(u)-\tau|^{2}\right)\left(s_{T}, t\right) d t \leq \frac{\|H\|}{T-1} .
$$

Gauge transforming the solution at $s=s_{T}$ we may assume, without loss of generality, that

$$
\Psi_{T}\left(s_{T}, t\right)=: \xi_{T}, \quad\left|\xi_{T}\right| \leq c,
$$

where $c$ is the diameter of $\mathrm{G}$ with respect to our biinvariant metric. Namely, choose $g: \mathbb{R} / \mathbb{Z} \rightarrow \mathrm{G}$ such that

$$
\partial_{t} g(t)+\Psi\left(s_{T}, t\right) g(t)=0, \quad g(0)=\mathbb{1} .
$$


Then write $g(1)^{-1}=\exp \left(\xi_{T}\right)$, where $\left|\xi_{T}\right| \leq c$, and gauge transform $u_{T}$ and $\Psi_{T}$ by the product $g(t) \exp \left(t \xi_{T}\right)$. Now (i.e. after gauge transforming) define $x_{T}: \mathbb{R} / \mathbb{Z} \rightarrow M$ by

$$
x_{T}(t):=u_{T}\left(s_{T}, t\right)
$$

Then

$$
\lim _{T \rightarrow \infty} \int_{0}^{1}\left(\left|\dot{x}_{T}(t)+L_{x_{T}(t)} \xi_{T}+X_{H_{t}}\left(x_{T}(t)\right)\right|^{2}+\left|\mu\left(x_{T}(t)\right)-\tau\right|^{2}\right) d t=0 .
$$

This shows that the $L^{2}$ norm of $\dot{x}_{T}$ is bounded and so $x_{T}$ is bounded and equicontinuous. Hence, by the Arzela-Ascoli theorem, there exists a sequence $T_{i} \rightarrow \infty$ such that $x_{T_{i}}$ converges uniformly, $\dot{x}_{T_{i}}$ converges weakly in $L^{2}$, and $\xi_{T_{i}}$ converges in $\mathfrak{g}$. The limit $(x, \xi)$ is the required solution of (47). Since $x_{T}$ is contractible for every $T$, so is $x$. This proves the theorem for compactly supported Hamiltonian functions. The general case follows by cutting off the Hamiltonian function outside of $\mu^{-1}(\tau)$.

\section{Weighted projective space.}

Consider the symplectic manifold $M=\mathbb{C}^{n}$ with the standard symplectic form and the $S^{1}$-action

$$
\lambda x=\left(\lambda^{\ell_{1}} x_{1}, \ldots, \lambda^{\ell_{n}} x_{n}\right),
$$

where $\ell_{1}, \ldots, \ell_{n}$ are positive integers. Then a moment map is given by

$$
\mu_{\ell}(x)=-\frac{i}{2} \sum_{\nu=1}^{n} \ell_{\nu}\left|x_{\nu}\right|^{2} .
$$

Suppose that $\Sigma$ has genus $g$ and let $P \rightarrow \Sigma$ be an $S^{1}$-bundle of degree $d$. Consider the complex line bundle

$$
L:=P \times_{S^{1}} \mathbb{C} \rightarrow \Sigma,
$$

where $S^{1}$ acts on $P \times \mathbb{C}$ by

$$
\lambda^{*}(p, \zeta)=\left(p \lambda, \lambda^{-1} \zeta\right)
$$

Then the symplectic vortex equations (1) with $\mu=\mu_{\ell}$ given by (52) can be written in the form

$$
\bar{\partial}_{A} u_{\nu}=0, \quad * i F_{A}+\sum_{\nu=1}^{n} \frac{\ell_{\nu}\left|u_{\nu}\right|^{2}}{2}=\tau,
$$


where $u_{\nu}$ is a section of $L^{\otimes \ell_{\nu}}$ for $\nu=1, \ldots, n$ and $A \in \mathcal{A}(L)$ is a Hermitian connection on $L$. Let us denote by $\mathcal{M}_{d, g}$ the space of gauge equivalence classes of solutions $\left(u_{1}, \ldots, u_{n}, A\right)$ of (53). The moduli space is nonempty only if

$$
\tau>\frac{2 \pi d}{\operatorname{Vol}(\Sigma)}
$$

Moreover, $\mathcal{M}_{d, g}$ has virtual dimension

$$
\operatorname{dim} \mathcal{M}_{d, g}=2\left(d \sum_{\nu=1}^{n} \ell_{\nu}-(n-1)(g-1)\right)=: 2 m .
$$

For $d$ sufficiently large the dimension is positive. We write $\Phi_{d, g}^{\mathbb{C}^{n}, \mu_{\ell}}:=$ $\Phi_{B, \Sigma}^{\mathbb{C}^{n}, \mu_{\ell}+i \tau}$ for the invariant in the nontrivial chamber. Let $c \in H_{S^{1}}^{2}\left(\mathbb{C}^{n} ; \mathbb{Z}\right) \cong$ $H^{2}\left(\mathrm{~B} S^{1} ; \mathbb{Z}\right) \cong \mathbb{Z}$ denote the positive generator.

Theorem 9.1. Assume

$$
m:=\sum_{\nu=1}^{n}\left(d \ell_{\nu}-g+1\right)+g-1 \geq 0 .
$$

Then, for $\alpha=0, \beta=c^{m}$, and $\gamma=\mathrm{pt}$, the invariant is

$$
\Phi_{d, g}^{\mathbb{C}^{n}, \mu_{\ell}}\left(0, c^{m} ; \mathrm{pt}\right)=\left(\sum_{\nu=1}^{n} \ell_{\nu}\right)^{g} \prod_{\nu=1}^{n} \ell_{\nu}^{-d \ell_{\nu}+g-1} .
$$

In the case $\ell_{\nu}=1$ and $d>2 g-2$ Theorem 9.1 was proved by BertramDaskalopoulos-Wentworth [2]. In this case the invariant $\Phi_{d, g}^{\mathbb{C}^{n}, \mu_{\ell}}\left(0, c^{m} ; \mathrm{pt}\right)$ corresponds to the Gromov-Witten invariant given by counting holomorphic maps $u: \Sigma \rightarrow \mathbb{C} P^{n-1}$ of degree $d$ (with a fixed complex structure on $\Sigma$ and a generic Hamiltonian perturbation) that pass at $m$ distinct specified points on $\Sigma$ through $m$ specified hyperplanes in $\mathbb{C} P^{n-1}$. For a proof of this correspondence in the case $\Sigma=S^{2}$ see [15]. We emphasize that in the higher genus case the Hamiltonian perturbation is needed in order to destroy the constant holomorphic maps $\Sigma \rightarrow \mathbb{C} P^{n-1}$ which are not regular. If one wants to work with the unperturbed Cauchy-Riemann equations one has to work with stable maps.

Proof of Theorem 9.1. We simplify (53) and consider instead the equations

$$
\bar{\partial}_{A} u_{\nu}=0, \quad * i F_{A}=\frac{2 \pi d}{\operatorname{Vol}(\Sigma)}, \quad \sum_{\nu=1}^{n} \ell_{\nu}\left\|u_{\nu}\right\|_{L^{2}}^{2}=1
$$


for $u_{\nu} \in \Omega^{0}\left(\Sigma, L^{\otimes \ell_{\nu}}\right)$ and $A \in \mathcal{A}(L)$. There are two ways to establish the correspondence between equations (53) and (54). One can use the action of the complexified gauge group and the Kazdan-Warner equation, or one can show that the corresponding $S^{1}$-moduli problems are homotopic. We use the latter approach. Consider the 1-parameter family of equations

$$
\bar{\partial}_{A} u_{\nu}=0, \quad * i F_{A}-\frac{2 \pi d}{\operatorname{Vol}(\Sigma)}=\frac{\varepsilon}{2}\left(\frac{1}{\operatorname{Vol}(\Sigma)}-\sum_{\nu=1}^{n} \ell_{\nu}\left|u_{\nu}\right|^{2}\right)
$$

for $0 \leq \varepsilon \leq 1$. For $\varepsilon=0$ this equation is equivalent to (54) and for $\varepsilon=1$ it is equivalent to $(53)$ with $\tau=(2 \pi d+1 / 2) / \operatorname{Vol}(\Sigma)$. Note, in particular, that $\sum_{\nu} \ell_{\nu}\left\|u_{\nu}\right\|_{L^{2}}^{2}=1$ for every $\varepsilon>0$ and every solution of (55). Thus we may formulate the $S^{1}$-moduli problems as follows. We shall not bother with Sobolev completions and formulate the problems in terms of smooth sections.

Fix a point $z_{0} \in \Sigma$ and consider the based gauge group

$$
\mathcal{G}_{0}:=\left\{g \in C^{\infty}\left(\Sigma, S^{1}\right) \mid g\left(z_{0}\right)=1\right\} .
$$

Define $\mathcal{B}$ by

$$
\begin{aligned}
& \mathcal{B}:=\left\{\left(A, u_{1}, \ldots, u_{n}\right) \mid A \in \mathcal{A}(L),\right. \\
&\left.u_{\nu} \in \Omega^{0}\left(\Sigma, L^{\otimes \ell_{\nu}}\right), \sum_{\nu=1}^{n} \ell_{\nu}\left\|u_{\nu}\right\|_{L^{2}}^{2}=1\right\} / \mathcal{G}_{0} .
\end{aligned}
$$

The bundle $\mathcal{H} \rightarrow \mathcal{B}$ has fibres

$$
\mathcal{H}_{A, u}:=\Omega^{0,1}\left(\Sigma, L^{\otimes \ell_{1}}\right) \oplus \cdots \oplus \Omega^{0,1}\left(\Sigma, L^{\otimes \ell_{n}}\right) \oplus \Omega_{0}^{0}(\Sigma)
$$

over $[A, u]=\left[A, u_{1}, \ldots, u_{n}\right] \in \mathcal{B}$, where $\Omega_{0}^{0}(\Sigma)$ denotes the space of smooth real valued functions of mean value zero. The section $\mathcal{S}_{\varepsilon}: \mathcal{B} \rightarrow \mathcal{H}$ is given by

$$
\begin{aligned}
\mathcal{S}_{\varepsilon}(A, u):=\left(\bar{\partial}_{A} u_{1}, \ldots, \bar{\partial}_{A} u_{n}, * i F_{A}-\right. & \frac{2 \pi d}{\operatorname{Vol}(\Sigma)} \\
& \left.-\frac{\varepsilon}{2}\left(\frac{1}{\operatorname{Vol}(\Sigma)}-\sum_{\nu=1}^{n} \ell_{\nu}\left|u_{\nu}\right|^{2}\right)\right) .
\end{aligned}
$$

With appropriate Sobolev completions this gives rise to a homotopy of regular $S^{1}$-moduli problems $\left(\mathcal{B}, \mathcal{H}, \mathcal{S}_{\varepsilon}\right)$. In fact, if $d \ell_{\nu}>2 g-2$ for every $\nu$ then, 
by Serre duality, $\mathcal{S}_{\varepsilon}$ is transverse to the zero section for every $\varepsilon$ and so the zero sets of $\mathcal{S}_{\varepsilon}$ give rise to a (trivial) cobordism from the moduli space of solutions of (54) to the moduli space of solutions of (53). In any case (even without $d \ell_{\nu}>2 g-2$ ) it follows from the (Homotopy) axiom for the Euler class that our invariant is given by

$$
\Phi_{d, g}^{\mathbb{C}^{n}, \mu_{\ell}}\left(c^{m}\right)=\chi^{\mathcal{B}, \mathcal{H}, \mathcal{S}_{1}}\left(\pi^{*} c^{m}\right)=\chi^{\mathcal{B}, \mathcal{H}, \mathcal{S}_{0}}\left(\pi^{*} c^{m}\right)
$$

where $\pi: \mathcal{B} \times{ }_{S^{1}} \mathrm{E} S^{1} \rightarrow \mathrm{B} S^{1}$ denotes the obvious projection. We shall now compute the last term in (56) using a localization formula for circle actions and the index theorem for families.

Fix a reference connection $A_{0} \in \mathcal{A}(L)$ and consider the space

$$
\mathcal{A}^{\text {coul }}:=\left\{A \in \mathcal{A}(L) \mid * i F_{A}=\frac{2 \pi d}{\operatorname{Vol}(\Sigma)}, d^{*}\left(A-A_{0}\right)=0\right\}
$$

of projectively flat connections in Coulomb gauge relative to $A_{0}$. The group

$$
\mathcal{G}^{\text {coul }}:=\left\{g \in C^{\infty}\left(\Sigma, S^{1}\right) \mid d^{*}\left(g^{-1} d g\right)=0\right\}
$$

of harmonic gauge transformations acts on $\mathcal{A}^{\text {coul }}$ and the quotient $\mathcal{A}^{\text {coul }} / \mathcal{G}^{\text {coul }}$ is diffeomorphic to the torus $\mathbb{T}^{2 g}$ (the Jacobian of degree $d$ line bundles over $\Sigma$ ). An explicit diffeomorphism can be constructed as follows. Choose $2 g$ embedded loops $\gamma_{1}, \ldots, \gamma_{2 g}$ in $\Sigma$ such that

$$
\gamma_{j} \cdot \gamma_{g+j}=1
$$

and $\gamma_{j} \cdot \gamma_{j^{\prime}}=0$ for $j^{\prime} \neq j \pm g$. Choose a dual basis $\alpha_{j}:=\operatorname{PD}\left(\gamma_{j}\right) \in H^{1}(\Sigma)$ of the space of harmonic 1-forms so that

$$
\int_{\gamma} \alpha_{j}=\gamma_{j} \cdot \gamma, \quad \int_{\Sigma} \alpha_{j} \wedge \alpha_{g+j}=1
$$

Let $\mathbb{Z}^{2 g} \rightarrow \mathcal{G}^{\text {coul }}: k \mapsto g_{k}$ be a group homomorphism such that

$$
\frac{1}{2 \pi i} \int_{\gamma_{j}} g_{k}^{-1} d g_{k}=k_{j}
$$

for every $k \in \mathbb{Z}^{2 g}$ and every $j \in\{1, \ldots, 2 g\}$. Then the map $\mathbb{R}^{2 g} \rightarrow \mathcal{A}^{\text {coul }}$ : $t \mapsto A_{t}$, defined by

$$
A_{t}:=A_{0}+\sum_{j=1}^{2 g} 2 \pi i t_{j} \alpha_{j}
$$


descends to a diffeomorphism $\mathbb{T}^{2 g} \rightarrow \mathcal{A}^{\text {coul }} / \mathcal{G}^{\text {coul }}$. Note that

$$
A_{t+k}=g_{k}^{*} A_{t}
$$

for $t \in \mathbb{R}^{2 g}$ and $k \in \mathbb{Z}^{2 g}$. Now consider the action of $\mathbb{Z}^{2 g}$ on $\mathbb{R}^{2 g} \times L$ via

$$
k \cdot(t,[p, \zeta]):=\left(t+k,\left[p, g_{k}(p)^{-1} \zeta\right]\right) .
$$

This action gives rise to a universal line bundle

$$
\mathbb{L}:=\frac{\mathbb{R}^{2 g} \times L}{\mathbb{Z}^{2 g}} \rightarrow \mathbb{T}^{2 g} \times \Sigma .
$$

For any integer $k \in \mathbb{Z}$ we denote by $\mathbb{L}^{k}=\mathbb{L} \otimes \cdots \otimes \mathbb{L}$ the $k$ th tensor power of $\mathbb{L}$. From now on we denote by $t$ an equivalence class in $\mathbb{T}^{2 g}=\mathbb{R}^{2 g} / \mathbb{Z}^{2 g}$. For $t \in \mathbb{T}^{2 g}$ denote by $\mathbb{L}_{t}^{k} \rightarrow \Sigma$ the restriction of $\mathbb{L}^{k}$ to $t \times \Sigma$. By (57), the bundle $\mathbb{L}_{t}^{k}$ is equipped with a connection $A_{t}^{k}$ and hence with a Cauchy-Riemann operator

$$
\bar{\partial}_{t}^{k}: \Omega^{0}\left(\Sigma, \mathbb{L}_{t}^{k}\right) \rightarrow \Omega^{0,1}\left(\Sigma, \mathbb{L}_{t}^{k}\right) .
$$

Denote the topological index (as a $K$-theory class) of this family of CauchyRiemann operators by

$$
\mathcal{I N} \mathcal{D}^{k}:=\bigcup_{t}\{t\} \times \operatorname{ker} \bar{\partial}_{t}^{k} \ominus \operatorname{coker} \bar{\partial}_{t}^{k} \in K\left(\mathbb{T}^{2 g}\right) .
$$

Now consider the vector bundle

$$
\mathbb{E}:=\mathbb{L}^{\ell_{1}} \oplus \cdots \oplus \mathbb{L}^{\ell_{n}} \rightarrow \mathbb{T}^{2 g} \times \Sigma .
$$

For $t \in \mathbb{T}^{2 g}$ denote by $\mathbb{E}_{t}$ the restriction of $\mathbb{E}$ to $\{t\} \times \Sigma$ and by

$$
\bar{\partial}_{t}: \Omega^{0}\left(\Sigma, \mathbb{E}_{t}\right) \rightarrow \Omega^{0,1}\left(\Sigma, \mathbb{E}_{t}\right)
$$

the corresponding Cauchy-Riemann operator. The $L^{2}$-norm of a section $u \in \Omega^{0}\left(\Sigma, \mathbb{E}_{t}\right)$ is given by

$$
\|u\|_{L^{2}}:=\sqrt{\sum_{\nu=1}^{n} \ell_{\nu}\left\|u_{\nu}\right\|_{L^{2}}^{2}}
$$

where the sections $u_{\nu} \in \Omega^{0}\left(\Sigma, \mathbb{L}_{t}^{\ell_{\nu}}\right)$ denote the components of $u$. This gives rise to an $S^{1}$-moduli problem as follows. Define

$$
\mathbb{B}:=\left\{(t, u) \mid t \in \mathbb{T}^{2 g}, u_{\nu} \in \Omega^{0}\left(\Sigma, \mathbb{E}_{t}\right),\|u\|_{L^{2}}=1\right\} .
$$


The circle $S^{1}$ acts on $\mathbb{B}$ by

$$
\lambda^{*}\left(t, u_{1}, \ldots, u_{n}\right):=\left(t, \lambda^{-\ell_{1}} u_{1}, \ldots, \lambda^{-\ell_{n}} u_{n}\right)
$$

for $\lambda \in S^{1}$. The bundle $\mathbb{H} \rightarrow \mathbb{B}$ has fibres

$$
\mathbb{H}_{t, u}:=\Omega^{0,1}\left(\Sigma, \mathbb{L}_{t}^{\ell_{1}}\right) \oplus \cdots \oplus \Omega^{0,1}\left(\Sigma, \mathbb{L}_{t}^{\ell_{n}}\right)=\Omega^{0,1}\left(\Sigma, \mathbb{E}_{t}\right)
$$

and the section $\mathbb{S}: \mathbb{B} \rightarrow \mathbb{H}$ is given by

$$
\mathbb{S}(t, u):=\bar{\partial}_{t} u \text {. }
$$

The obvious embeddings define a morphism from $(\mathbb{B}, \mathbb{H}, \mathbb{S})$ to $\left(\mathcal{B}, \mathcal{H}, \mathcal{S}_{0}\right)$ and so, by the (Functoriality) axiom for $S^{1}$-moduli problems and (56), we have

$$
\Phi_{d, g}^{\mathbb{C}^{n}, \mu_{\ell}}\left(c^{m}\right)=\chi^{\mathbb{B}, \mathbb{H}, \mathbb{S}}\left(\pi_{\mathbb{B}}^{*} c^{m}\right),
$$

where $\pi_{\mathbb{B}}: \mathbb{B} \times_{S^{1}} \mathrm{E} S^{1} \rightarrow \mathrm{B} S^{1}$ is the projection. Here the action of $S^{1}$ on $\mathbb{B} \times \mathrm{E} S^{1}$ is given by $\lambda^{*}(t, u, e)=\left(t, \lambda^{-\ell_{1}} u_{1}, \ldots, \lambda^{-\ell_{n}} u_{n}, \lambda^{-1} e\right)$.

Now the $S^{1}$-moduli problem $(\mathbb{B}, \mathbb{H}, \mathbb{S})$ satisfies the hypotheses of the localization formula for circle actions in [7, Theorem 11.1] and we get

$$
\Phi_{d, g}^{\mathbb{C}^{n}, \mu_{\ell}}\left(c^{m}\right)=\int_{\mathbb{T}^{2 g}} \frac{1}{\prod_{\nu=1}^{n} c\left(\mathcal{I N} \mathcal{D}^{\ell_{\nu}}, \ell_{\nu}\right)} .
$$

Here $c(\mathcal{I N D}, \cdot)$ denotes the Chern series of the $K$-theory class $\mathcal{I N D} \in$ $K\left(\mathbb{T}^{2 g}\right)$. It is defined by

$$
c(\mathcal{I N} \mathcal{D}(\mathcal{D}), \eta):=\sum_{j \geq 0} \eta^{\operatorname{index}(\mathcal{D})-j} c_{j}(\mathcal{I N} \mathcal{D}(\mathcal{D}))
$$

where $\operatorname{index}(\mathcal{D}):=\operatorname{dim} \operatorname{ker} \mathcal{D}-\operatorname{dim}$ coker $\mathcal{D}$ is the Fredholm index.

The right hand side of (58) can be computed by means of the AtiyahSinger index theorem for families (see [31]). It asserts that

$$
\operatorname{ch}\left(\mathcal{I N} \mathcal{D}^{k}\right)=\int_{\Sigma} \operatorname{td}(T \Sigma) \operatorname{ch}\left(\mathbb{L}^{k}\right) \in H^{*}\left(\mathbb{T}^{2 g}\right)
$$

Here the Todd class and the Chern character of a line bundle $L$ with first Chern class $c_{1}(L)=x$ are defined as

$$
\operatorname{td}(L):=\frac{x}{1-e^{-x}}, \quad \operatorname{ch}(L):=e^{x} .
$$


The Todd class of $T \Sigma$ is given by

$$
\operatorname{td}(T \Sigma)=1+(1-g) \sigma
$$

where $\sigma \in H^{2}(\Sigma ; \mathbb{Z})$ denotes the positive generator, represented by a volume form with respect to which $\Sigma$ has volume one. By Lemma 7.9, the first Chern class of $\mathbb{L}^{k}$ is given by

$$
c_{1}\left(\mathbb{L}^{k}\right)=k\left(\sum_{j=1}^{2 g} \alpha_{j} \wedge \tau_{j}+d \sigma\right)
$$

where $\alpha_{j}=\operatorname{PD}\left(\gamma_{j}\right) \in H^{1}(\Sigma)$ and $\tau_{j}:=\left[d t_{j}\right] \in H^{1}\left(\mathbb{T}^{2 g} ; \mathbb{Z}\right)$. Let us denote by

$$
\Omega:=\sum_{j=1}^{g} \tau_{j} \wedge \tau_{g+j}
$$

the cohomology class of the standard symplectic form on $\mathbb{T}^{2 g}$. Then the Chern character of $\mathbb{L}^{k}$ is given by

$$
\begin{aligned}
\operatorname{ch}\left(\mathbb{L}^{k}\right) & =1+c_{1}\left(\mathbb{L}^{k}\right)+\frac{c_{1}\left(\mathbb{L}^{k}\right)^{2}}{2} \\
& =1+d k \sigma-k^{2} \sigma \wedge \Omega+k \sum_{j=1}^{2 g} \alpha_{j} \wedge \tau_{j} .
\end{aligned}
$$

Hence

$$
\operatorname{td}(T \Sigma) \operatorname{ch}\left(\mathbb{L}^{k}\right)=1+(d k+1-g) \sigma-k^{2} \sigma \wedge \Omega+k \sum_{j=1}^{2 g} \alpha_{j} \wedge \tau_{j},
$$

and integration over the fibre gives

$$
\operatorname{ch}\left(\mathcal{I N} \mathcal{D}^{k}\right)=\int_{\Sigma} \operatorname{td}(T \Sigma) \operatorname{ch}\left(\mathbb{L}^{k}\right)=d k+1-g-k^{2} \Omega .
$$

This implies (cf. [31])

$$
c_{1}\left(\mathcal{I N} \mathcal{D}^{k}\right)=-k^{2} \Omega, \quad c_{j}\left(\mathcal{I N} \mathcal{D}^{k}\right)=\frac{1}{j !} c_{1}\left(\mathcal{I N} \mathcal{D}^{k}\right)^{j},
$$

and so the Chern series of $\mathcal{I N D} \mathcal{D}^{k}$ is given by

$$
c\left(\mathcal{I N} \mathcal{D}^{k}, \eta\right)=\eta^{d k+1-g} \exp \left(-\eta^{-1} k^{2} \Omega\right) .
$$


Since the integral of $\Omega^{g} / g$ ! over $\mathbb{T}^{2 g}$ is one, we obtain from (58) that

$$
\begin{aligned}
& \Phi_{d, g}^{\mathbb{C}^{n}, \mu_{\ell}}\left(c^{m}\right)=\int_{\mathbb{T}^{2 g}} \frac{1}{\prod_{\nu=1}^{n} c\left(\mathcal{I} \mathcal{N} \mathcal{D}^{\ell_{\nu}}, \ell_{\nu}\right)} \\
& =\prod_{\nu=1}^{n} \ell_{\nu}^{-d \ell_{\nu}+g-1} \int_{\mathbb{T}^{2 g}} \frac{1}{\prod_{\nu=1}^{n} \exp \left(-\ell_{\nu} \Omega\right)} \\
& =\prod_{\nu=1}^{n} \ell_{\nu}^{-d \ell_{\nu}+g-1} \int_{\mathbb{T}^{2 g}} \prod_{\nu=1}^{n} \exp \left(\ell_{\nu} \Omega\right) \\
& =\prod_{\nu=1}^{n} \ell_{\nu}^{-d \ell_{\nu}+g-1} \int_{\mathbb{T}^{2 g}} \exp \left(\sum_{\nu=1}^{n} \ell_{\nu} \Omega\right) \\
& =\left(\sum_{\nu=1}^{n} \ell_{\nu}\right)^{g} \prod_{\nu=1}^{n} \ell_{\nu}^{-d \ell_{\nu}+g-1} \text {. }
\end{aligned}
$$

This proves the theorem.

\section{Seiberg-Witten invariants.}

In this section we explain how the Seiberg-Witten invariants of a product

$$
X=\Sigma \times S
$$

are related to our invariants is the case where either $S$ or $\Sigma$ is a sphere. The relation will be established by considering the symplectic vortex equations over $\Sigma$ with a suitable target manifold $M_{S}$. The space $M_{S}$ is a symplectic manifold with a circle action and the quotient $M_{S} / / S^{1}$ is the $d$-fold symmetric product of $S$. In fact, the space $M_{S}$ itself consists of (gauge equivalence classes of) solutions to the vortex equations over $S$. It is a special case of the socalled master space for the vortex equations constructed in [5]. Here is how this works.

Let $\left(S, J_{S}, \mathrm{dvol}_{S}\right)$ be a compact Riemann surface of genus $g_{S}$ and $L \rightarrow S$ be a complex Hermitian line bundle of degree

$$
\operatorname{deg}(L)=d>2 g_{S}-2 .
$$

For a Hermitian connection $A \in \mathcal{A}(L)$ and a section $\Theta \in \Omega^{0}(S, L)$ consider the vortex equations

$$
\bar{\partial}_{A} \Theta=0, \quad * i F_{A}+\frac{|\Theta|^{2}}{2}-\frac{1}{2 \operatorname{Vol}(S)} \int_{S}|\Theta|^{2} \operatorname{dvol}_{S}=\frac{2 \pi d}{\operatorname{Vol}(S)} .
$$


The gauge group $\mathcal{G}_{S}:=C^{\infty}\left(S, S^{1}\right)$ acts on the space of solutions of (59) and the action is free whenever $\Theta \neq 0$. Fix a point $x_{0} \in S$ and consider the homomorphism $\rho_{0}: \mathcal{G}_{S} \rightarrow S^{1}$ defined by

$$
\rho_{0}(g):=\exp \left(-\xi\left(x_{0}\right)\right) g\left(x_{0}\right), \quad d^{*} d \xi=d^{*}\left(g^{-1} d g\right), \quad \int_{S} \xi \operatorname{dvol}_{S}=0 .
$$

Its kernel is the subgroup $\mathcal{G}_{S 0} \subset \mathcal{G}_{S}$ of all smooth maps $g: S \rightarrow S^{1}$ of the form $g=g_{0} \exp (\xi)$, where $g_{0}: S \rightarrow S^{1}$ is a harmonic map that vanishes at $x_{0}$ and $\xi: S \rightarrow i \mathbb{R}$ has mean value zero. Thus the Lie algebra of $\mathcal{G}_{S 0}$ is the space of imaginary valued functions of mean value zero:

$$
\operatorname{Lie}\left(\mathcal{G}_{S 0}\right)=\Omega_{0}^{0}(S, i \mathbb{R}):=\left\{\xi \in \Omega^{0}(S, i \mathbb{R}) \mid \int_{S} \xi \mathrm{dvol}_{S}=0\right\}
$$

Let us denote the space of solutions of (59) by

$$
\tilde{M}_{S}:=\left\{(A, \Theta) \in \mathcal{A}(L) \times \Omega^{0}(S, L) \mid A \text { and } \Theta \text { satisfy (59) }\right\}
$$

and the quotient by the action of $\mathcal{G}_{S 0}$ by

$$
M_{S}:=\tilde{M}_{S} / \mathcal{G}_{S 0}
$$

The tangent space of $M_{S}$ at a pair $(A, \Theta)$ can be identified with the space of all pairs $(\alpha, \theta) \in \Omega^{1}(S, i \mathbb{R}) \times \Omega^{0}(S, L)$ that satisfy the linearized equation

$$
\begin{aligned}
& \bar{\partial}_{A} \theta+\alpha^{0,1} \Theta=0, \\
& * i d \alpha+\langle\Theta, \theta\rangle-\frac{1}{\operatorname{Vol}(S)} \int_{S}\langle\Theta, \theta\rangle \mathrm{dvol}_{S}=0, \\
&-d^{*} \alpha+i\langle i \Theta, \theta\rangle-\frac{1}{\operatorname{Vol}(S)} \int_{S} i\langle i \Theta, \theta\rangle \mathrm{dvol}_{S}=0 .
\end{aligned}
$$

Here the last equation asserts that the pair $(\alpha, \theta)$ belongs to the local slice of the $\mathcal{G}_{S 0}$-action, i.e. it is $L^{2}$ orthogonal to the $\mathcal{G}_{S 0}$-orbit of the pair $(A, \Theta)$. The left hand side of $(60)$ defines a surjective Fredholm operator from $\Omega^{0}(S, L) \oplus \Omega^{1}(S, i \mathbb{R})$ to $\Omega^{0,1}(S, L) \oplus \Omega_{0}^{0}(S, \mathbb{C})$ whenever $\Theta \neq 0$. The condition $d>2 g_{S}-2$ guarantees surjectivity also in the case $\Theta=0$. So in this case $M_{S}$ is a manifold of dimension $2 d+2$. Unfortunately, the case $d>2 g_{S}-2$ is only interesting when $S$ has genus zero (see Remark 10.5 below). If $d \leq 2 g_{S}-2$ the space $M_{S}$ has singularities at the points where $\Theta=0$. In the case $d \leq 2 g_{S}-2$ and $g_{S}>0$ the space $M_{S}$ can be desingularized by a blowup construction, however this leads to holomorphic spheres in the ambient space $M_{S}$ and so our theory does not apply in its present form. 
A symplectic form $\omega_{S}$ on $M_{S}$ is given by

$$
\omega_{S}\left((\alpha, \theta),\left(\alpha^{\prime}, \theta^{\prime}\right)\right):=-\int_{S} \alpha \wedge \alpha^{\prime}+\int_{S}\left\langle i \theta, \theta^{\prime}\right\rangle \operatorname{dvol}_{S}
$$

for two solutions $(\alpha, \theta)$ and $\left(\alpha^{\prime}, \theta^{\prime}\right)$ of $(60)$. One can think of $M_{S}$ as the symplectic quotient of the space of all pairs $(A, \Theta)$ that satisfy $\bar{\partial}_{A} \Theta=0$ by the (Hamiltonian) $\mathcal{G}_{S 0}$-action. The linear map

$$
(\alpha, \theta) \mapsto(* \alpha, i \theta)
$$

on the space of solutions of (60) defines a complex structure $J_{S}$ on $M_{S}$ that is compatible with $\omega_{S}$. Thus $\left(M_{S}, \omega_{S}, J_{S}\right)$ is a Kähler manifold.

Now the circle $S^{1}$ acts on $M_{S}$ through the constant gauge transformations. This action is Hamiltonian with moment map

$$
\mu_{S}(A, \Theta)=-\frac{i}{2 \operatorname{Vol}(S)} \int_{S}|\Theta|^{2} \operatorname{dvol}_{S}
$$

The factor $1 / \operatorname{Vol}(S)$ arises from the fact that we identify the circle with the subgroup of $\mathcal{G}_{S}$ of constant gauge transformations and use the standard $L^{2}$ metric on $\operatorname{Lie}\left(\mathcal{G}_{S}\right)=\Omega^{0}(S, i \mathbb{R})$ to define the moment map as a function with values in the Lie algebra, and not its dual.

Let us recall some standard facts about the space $M_{S}$ (see $\left.[3,16]\right)$. There is a one-to-one correspondence between Hermitian connections $A \in \mathcal{A}(L)$ and holomorphic structures on $L$ via $A \mapsto \bar{\partial}_{A}$. Moreover, the KazdanWarner equation shows that every pair $(A, \Theta) \in \mathcal{A}(L) \times C^{\infty}(S, L)$ such that $\bar{\partial}_{A} \Theta=0$ is complex gauge equivalent to a solution of (59) by a gauge transformation of the form $g=e^{f}$ where $f: S \rightarrow \mathbb{R}$ has mean value zero (see Proposition A.3). Hence the space $M_{S}$ can be identified with the space of $\mathcal{G}_{S 0}^{c}$-gauge equivalence classes of the space of pairs $(A, \Theta)$ that satisfy $\bar{\partial}_{A} \Theta=0$. For $d>2 g_{S}-2$ it follows from Serre duality that this is a vector bundle over the Picard variety of holomorphic bundles of degree $d$ over $S$, $\operatorname{Pic}^{d}(S) \cong T^{2 g}$, with fibre $\mathbb{C}^{d+1-g_{S}}$. The circle acts trivially on the base and by the standard action on the fibres.

This shows that the triple $\left(M_{S}, \omega_{S}, \mu_{S}\right)$ satisfies hypotheses $(H 1-3)$, namely, $\mu_{S}$ is proper, the moment map is convex at infinity, and $\pi_{2}\left(M_{S}\right)=0$. Moreover every nonzero imaginary number is a regular value of $\mu_{S}$ and $S^{1}$ acts freely on the preimage under $\mu_{S}$. The quotient is nonempty if and only if the imaginary part is negative.

Remark 10.1. The symplectic quotient

$$
\bar{M}_{S}:=M_{S} / / S^{1}(-i / 2 \operatorname{Vol}(S))
$$


is a bundle over $T^{2 g}$ with fibre $\mathbb{C} P^{d-g_{S}}$. On the other hand, this quotient is the space of effective divisors of degree $d$ on $S$, so $\bar{M}_{S} \cong \operatorname{Sym}^{d}(S)$ is the $d$-fold symmetric product of $S$.

The next theorem states that the invariants of the triple $\left(M_{S}, \omega_{S}, \mu_{S}\right)$ for a Riemann surface $\Sigma$ agree with the Seiberg-Witten invariants of the product $\Sigma \times S$. We denote by $c \in H^{2}\left(\mathrm{~B} S^{1} ; \mathbb{Z}\right)$ the positive generator and by $\pi_{S}: M_{S} \times_{S^{1}} \mathrm{E} S^{1} \rightarrow \mathrm{B} S^{1}$ the obvious projection. For a nonnegative integer $k \in Z$ denote by

$$
\Phi_{k, \Sigma}^{M_{S}, \mu_{S}}:=\Phi_{k, \Sigma}^{M_{S}, \mu_{S}+i / 2}
$$

the invariant in the nontrivial chamber. Let

$$
E_{k, d} \rightarrow \Sigma \times S
$$

be the complex line bundle which has degree $k$ over $\Sigma$ and degree $d$ over $S$, and denote by $\gamma_{k, d} \in \operatorname{Spin}^{c}(\Sigma \times S)$ the $\operatorname{spin}^{c}$ structure obtained by twisting the standard $\operatorname{spin}^{c}$ structure $\gamma_{0}$ (associated to the complex structure) by $E_{k, d}$. If both $\Sigma$ and $S$ have positive genus the four-manifold $\Sigma \times S$ has $b^{+}>1$ and carries a well-defined Seiberg-Witten invariant

$$
S W_{\Sigma \times S}: \operatorname{Spin}^{c}(\Sigma \times S) \rightarrow \mathbb{Z} .
$$

If $\Sigma$ or $S$ is the sphere then $b^{+}=1$, so there are two chambers for the Seiberg-Witten invariants. In this case we denote by $\mathrm{SW}_{\Sigma \times S}$ the SeibergWitten invariant in the positive chamber, where "positive" is defined in the proof of Theorem 10.2 below. A result similar to the next theorem was proved in [27].

Theorem 10.2. Let $S$ and $\Sigma$ be a compact Riemann surfaces of genera $g_{S}$ and $g_{\Sigma}$, respectively, and $k, d$ be nonnegative integers such that

$$
m:=d\left(1-g_{\Sigma}\right)+k\left(1-g_{S}\right)+d k \geq 0, \quad d>2 g_{S}-2 .
$$

Then

$$
\Phi_{k, \Sigma}^{M_{S}, \mu_{S}}\left(0, \pi_{S}^{*} c^{m} ; \mathrm{pt}\right)=\mathrm{SW}_{\Sigma \times S}\left(\gamma_{k, d}\right) .
$$

If $m<0$ then both invariants are zero.

Corollary 10.3 ([21, 28]). Let $S$ be the Riemann sphere, $\Sigma$ a compact Riemann surface of genus $g_{\Sigma}$ and $k, d$ be nonnegative integers such that

$$
m:=d\left(1-g_{\Sigma}\right)+(d+1) k \geq 0 .
$$

Then

$$
\operatorname{SW}_{\Sigma \times S}\left(\gamma_{k, d}\right)=(d+1)^{g_{\Sigma}}
$$


Proof. Since $S$ is the Riemann sphere the manifold $M_{S}$ is diffeomorphic to $\mathbb{C}^{d+1}$ as a Kähler manifold with an $S^{1}$ action. Hence the result follows from Theorem 10.2 and Theorem 9.1 with $n=d+1$ and $\ell_{1}=\cdots=\ell_{n}=1$.

Corollary 10.4. Let $S$ and $\Sigma$ be a compact Riemann surfaces of genera $g_{S}$ and $g_{\Sigma}$, respectively, and $k, d$ be nonnegative integers such that

$$
m:=d\left(1-g_{\Sigma}\right)+k\left(1-g_{S}\right)+d k \geq 0, \quad d>2 g_{S}-2, \quad k>2 g_{\Sigma}-2 .
$$

Then

$$
\Phi_{k, \Sigma}^{M_{S}, \mu_{S}}\left(0, \pi_{S}^{*} c^{m} ; \mathrm{pt}\right)=\Phi_{d, S}^{M_{\Sigma}, \mu_{\Sigma}}\left(0, \pi_{\Sigma}^{*} c^{m} ; \mathrm{pt}\right)
$$

where $M_{S}$ is associated to a bundle of degree $d$ over $S$ via (59) and $M_{\Sigma}$ is defined analogously, with $S$ and $d$ replaced by $\Sigma$ and $k$.

Proof. Interchange the roles of $\Sigma$ and $S$ in the proof of Theorem 10.2.

Remark 10.5. The statements of Theorem 10.2 and Corollary 10.4 are only interesting when one of the two surfaces has genus zero. Otherwise both invariants are zero. To see this, suppose that both genera are positive. Then $\Sigma \times S$ is a minimal Kähler surface with $b^{+}>1$. It follows (see for example [31]) that the Seiberg-Witten invariant is nonzero only for the canonical $\operatorname{spin}^{c}$ structure and its dual, i.e. for $d=k=0$ or $d=2 g_{S}-2$ and $k=2 g_{\Sigma}-2$. These cases are excluded by our hypotheses.

Proof of Theorem 10.2. Our proof follows the argument outlined in [6]. The Seiberg-Witten equations for the $\operatorname{spin}^{c}$ structure $\gamma_{k, d}$ on $X:=\Sigma \times S$ have the form

$$
\begin{gathered}
\bar{\partial}_{B} \Theta_{0}+\bar{\partial}_{B}^{*} \Theta_{2}=0, \quad F_{B}^{0,2}-\left\langle\Theta_{0}, \Theta_{2}\right\rangle=0, \\
i\left(F_{B}\right)_{\Omega}+\frac{\left|\Theta_{0}\right|^{2}-\left|\Theta_{2}\right|^{2}}{2}=\tau
\end{gathered}
$$

where $\tau$ is a real number, $B \in \mathcal{A}(E)$ is a connection on $E:=E_{k, d}, \Theta_{0} \in$ $\Omega^{0,0}(X, E)$, and $\Theta_{2} \in \Omega^{0,2}(X, E)$. Here we denote by $\langle\cdot, \cdot\rangle$ a Hermitian inner product on $E$, i.e. the inner product takes values in $\mathbb{C}$, it is complex antilinear in the first variable and complex linear in the second variable. In the last term the function $\Omega^{2}(X, i \mathbb{R}) \rightarrow \Omega^{0}(X, i \mathbb{R}), \eta \mapsto \eta_{\Omega}$ is defined by

$$
\eta_{\Omega}:=*(\eta \wedge \Omega)
$$


where $p_{\Sigma}: X \rightarrow \Sigma$ and $p_{S}: X \rightarrow S$ denote the projections,

$$
\Omega:=p_{\Sigma}^{*} \operatorname{dvol}_{\Sigma}+p_{S}^{*} \operatorname{dvol}_{S} \in \Omega^{2}(X)
$$

denotes the symplectic form, and $*$ denotes the Hodge $*$-operator on $X$.

In the Kähler case it follows from (62) that either $\Theta_{0}$ or $\Theta_{2}$ vanishes. The positive chamber for the Seiberg-Witten invariants corresponds to the condition

$$
\tau>\frac{2 \pi k}{\operatorname{Vol}(\Sigma)}+\frac{2 \pi d}{\operatorname{Vol}(S)}
$$

In the Kähler case this condition implies $\Theta_{2}=0$.

The $S^{1}$-moduli problem associated to equations (62) and (63) is defined as follows. As in the proof of Theorem 9.1 we shall not explain the (obvious) Sobolev completions and describe the problem in terms of smooth data. Fix a point $\left(z_{0}, x_{0}\right) \in \Sigma \times S$ and denote by $\mathcal{G}_{X 0}$ the based gauge group of all smooth functions $g: X \rightarrow S^{1}$ such that the restriction of $g$ to $\left\{z_{0}\right\} \times S$ belongs to the subgroup $\mathcal{G}_{S 0} \subset \mathcal{G}_{S}$ determined by the point $x_{0}$ :

$$
\mathcal{G}_{X 0}:=\left\{g \in C^{\infty}\left(\Sigma \times S, S^{1}\right)|g|_{\left\{z_{0}\right\} \times S} \in \mathcal{G}_{S 0}\right\} .
$$

Then the base $\mathcal{B}^{\mathrm{SW}}$ is the quotient

$$
\mathcal{B}^{\mathrm{SW}}:=\frac{\left\{\left(B, \Theta_{0}, \Theta_{2}\right) \in \mathcal{A}(E) \times \Omega^{0}(X, E) \times \Omega^{0,2}(X, E) \mid(63)\right\}}{\mathcal{G}_{X 0}},
$$

the bundle $\mathcal{E}^{\mathrm{SW}} \rightarrow \mathcal{B}^{\mathrm{SW}}$ is given by

$$
\mathcal{E}^{\mathrm{SW}}:=\frac{\mathcal{B}^{\mathrm{SW}} \times\left(\Omega^{0,1}(X, E) \oplus \Omega^{0,2}(X)\right)}{\mathcal{G}_{X 0}}
$$

and the section $\mathcal{S}^{\mathrm{SW}}: \mathcal{B}^{\mathrm{SW}} \rightarrow \mathcal{E}^{\mathrm{SW}}$ is given by

$$
\mathcal{S}^{\mathrm{SW}}\left(B, \Theta_{0}, \Theta_{2}\right):=\left(\bar{\partial}_{B} \Theta_{0}+\bar{\partial}_{B}^{*} \Theta_{2}, F_{B}^{0,2}-\left\langle\Theta_{0}, \Theta_{2}\right\rangle\right) .
$$

With appropriate Sobolev completions this is a regular $S^{1}$-moduli problem in the sense of Definition 6.1 and the Seiberg-Witten invariant can be expressed in the form

$$
\mathrm{SW}_{\Sigma \times S}\left(\gamma_{k, d}\right)=\chi^{\mathcal{B}^{\mathrm{SW}}, \mathcal{E}^{\mathrm{SW}}, \mathcal{S}^{\mathrm{SW}}}\left(\pi_{S W}^{*} c^{m}\right),
$$

where $m=d\left(1-g_{\Sigma}\right)+k\left(1-g_{S}\right)+d k, \pi_{S W}: \mathcal{B}^{\mathrm{SW}} \times_{S^{1}} \mathrm{E} S^{1} \rightarrow \mathrm{B} S^{1}$ denotes the projection, and $c \in H^{2}\left(\mathrm{~B} S^{1} ; \mathbb{Z}\right)$ is the positive generator. 
Now let us examine the symplectic vortex equations with values in $M_{S}$. Let $P \rightarrow \Sigma$ be a principal $S^{1}$-bundle of degree $k$. Consider the associated bundle

$$
L_{P}:=P \times_{S^{1}} \mathbb{C} \rightarrow \Sigma
$$

and denote by

$$
E_{P}:=p_{\Sigma}^{*} L_{P} \otimes p_{S}^{*} L
$$

the corresponding bundle over $X=\Sigma \times S$, where $p_{\Sigma}: X \rightarrow \Sigma$ and $p_{S}$ : $X \rightarrow S$ denote the projections. In explicit terms it can be represented as the quotient

$$
E_{P}:=\frac{P \times L}{S^{1}}, \quad \lambda^{*}((z, p),(x, v)):=\left((z, p \lambda),\left(x, \lambda^{-1} v\right)\right) .
$$

This vector bundle has degree $k$ over $\Sigma$ and degree $d$ over $S$ and hence is isomorphic to $E$. Henceforth we shall drop the subscript $P$ and write $E:=E_{P}$.

The space $\mathcal{A}(P) \times C_{S^{1}}^{\infty}\left(P, M_{S}\right)$ embeds into the space $\mathcal{A}(E) \times \Omega^{0}(X, E)$ as follows. A connection $A_{\Sigma}$ on $P$ determines a connection $p_{\Sigma}^{*} A_{\Sigma}$ on $p_{\Sigma}^{*} L_{P}$. An $S^{1}$-equivariant function $u: P \rightarrow M_{S}$ consists of an $S^{1}$-invariant function $A: P \rightarrow \mathcal{A}(L)$ and an $S^{1}$-equivariant function $\Theta: P \rightarrow \Omega^{0}(S, L)$. The latter can be interpreted as a section of $E$ and the two connections together determine a connection

$$
p_{\Sigma}^{*} A_{\Sigma} \otimes 1+1 \otimes p_{S}^{*} A \in \mathcal{A}(E)
$$

To understand this correspondence better let us choose holomorphic local coordinates $s+i t \in U \subset \mathbb{C}$ on $\Sigma$ and a trivialization of $P$ along this coordinate chart. In such a trivialization the connection $A_{\Sigma}$ has the form $\Phi_{1} d s+\Psi_{1} d t$ where $\Phi_{1}, \Psi_{1}: U \rightarrow i \mathbb{R}$. The function $u$ is a map $U \rightarrow \mathcal{A}(L) \times \Omega^{0}(S, L)$ denoted by

$$
U \rightarrow \mathcal{A}(L):(s, t) \mapsto A(s, t), \quad U \rightarrow \Omega^{0}(S, L):(s, t) \mapsto \Theta(s, t)
$$

The corresponding connection on $E$ is given in this local frame by $A(s, t)+$ $\Phi_{1}(s, t) d s+\Psi_{1}(s, t) d t$. The pair $\left(A_{\Sigma}, u\right)$ satisfies equation (1) if and only if 
there exist functions $\Phi_{0}, \Psi_{0}: U \rightarrow \Omega_{0}^{0}(S, i \mathbb{R})$ such that, for all $s$ and $t$,

$$
\begin{aligned}
\bar{\partial}_{A} \Theta & =0 \\
\partial_{s} \Theta+\left(\Phi_{0}+\Phi_{1}\right) \Theta+i\left(\partial_{t} \Theta+\left(\Psi_{0}+\Psi_{1}\right) \Theta\right) & =0 \\
\partial_{s} A-d \Phi_{0}+*_{S}\left(\partial_{t} A-d \Psi_{0}\right) & =0 \\
*_{S} i F_{A}+\frac{1}{2}|\Theta|^{2}-\frac{1}{2 \operatorname{Vol}(S)} \int_{S}|\Theta|^{2} \operatorname{dvol}_{S} & =\frac{2 \pi d}{\operatorname{Vol}(S)}, \\
\lambda^{-2}\left(\partial_{s} \Psi_{1}-\partial_{t} \Phi_{1}\right)-\frac{i}{2 \operatorname{Vol}(S)} \int_{S}|\Theta|^{2} \operatorname{dvol}_{S} & =-i\left(\tau-\frac{2 \pi d}{\operatorname{Vol}(S)}\right) .
\end{aligned}
$$

Here $*_{S}$ denotes the Hodge $*$-operator on $S$ and $\lambda: U \rightarrow(0, \infty)$ represents the volume form $\lambda^{2} d s \wedge d t$ on $\Sigma$. The factor $\lambda^{-2}$ in the last term arises from the Hodge $*$-operator on $\Sigma$. The functions $\Phi_{0}$ and $\Psi_{0}$ are needed to project the terms in the second and third equation onto the quotient by the gauge group $\mathcal{G}_{S 0}$. Let us abbreviate

$$
\Phi:=\Phi_{0}+\Phi_{1}, \quad \Psi:=\Psi_{0}+\Psi_{1}
$$

Then (65) can be written in the form

$$
\begin{aligned}
\bar{\partial}_{A} \Theta & =0, \\
\partial_{s} \Theta+\Phi \Theta+i\left(\partial_{t} \Theta+\Psi \Theta\right) & =0, \\
\partial_{s} A-d \Phi+*_{S}\left(\partial_{t} A-d \Psi\right) & =0, \\
*_{S} i F_{A}+\frac{1}{2}|\Theta|^{2}-\frac{1}{2 \operatorname{Vol}(S)} \int_{S}|\Theta|^{2} \operatorname{dvol}_{S} & =\frac{2 \pi d}{\operatorname{Vol}(S)}, \\
\frac{i \lambda^{-2}}{\operatorname{Vol}(S)} \int_{S}\left(\partial_{s} \Psi-\partial_{t} \Phi\right) \mathrm{dvol}_{S}+\frac{1}{2 \operatorname{Vol}(S)} \int_{S}|\Theta|^{2} \operatorname{dvol}_{S} & =\tau-\frac{2 \pi d}{\operatorname{Vol}(S)}
\end{aligned}
$$

(cf. [6]). Now consider the connection

$$
B:=A(s, t)+\Phi(s, t) d s+\Psi(s, t) d t
$$

on $E$ and think of $\Theta$ as a section of $E$. Then the first two equations in (66) are equivalent to $\bar{\partial}_{B} \Theta=0$. The curvature of $B$ is the 2 -form

$$
F_{B}=F_{A}+d s \wedge\left(\partial_{s} A-d \Phi\right)+d t \wedge\left(\partial_{t} A-d \Psi\right)+\left(\partial_{s} \Psi-\partial_{t} \Phi\right) d s \wedge d t .
$$

The third equation in (66) asserts that $F_{B}^{0,2}=0$. The last two equations can be written in the form

$$
\frac{i \lambda^{-2}}{\operatorname{Vol}(S)} \int_{S}\left(\partial_{s} \Psi-\partial_{t} \Phi\right) \operatorname{dvol}_{S}+*_{S} i F_{A}+\frac{1}{2}|\Theta|^{2}=\tau,
$$


or in terms of the connection $B$,

$$
\frac{1}{\operatorname{Vol}(S)} \int_{S} * i F_{B}+* i\left(F_{B} \wedge \operatorname{dvol}_{\Sigma}\right)+\frac{|\Theta|^{2}}{2}=\tau .
$$

Here the integral denotes integration over the fibre. Hence a pair $\left(A_{\Sigma}, u\right) \in$ $\mathcal{A}(P) \times C_{S^{1}}^{\infty}\left(P, M_{S}\right)$ satisfies the symplectic vortex equation (1) if and only if the corresponding pair $(B, \Theta) \in \mathcal{A}(E) \times \Omega^{0}(X, E)$ satisfies equation (67) and

$$
\bar{\partial}_{B} \Theta=0, \quad F_{B}^{0,2}=0 .
$$

Integration of equation (67) over $\Sigma$ yields

$$
\frac{2 \pi k}{\operatorname{Vol}(\Sigma)}+\frac{2 \pi d}{\operatorname{Vol}(S)}+\frac{1}{2 \operatorname{Vol}(\Sigma)} \int_{\Sigma}|\Theta|^{2} \operatorname{dvol}_{\Sigma}=\tau
$$

If (64) holds then $\Theta \neq 0$ for every solution of (67). Hence equations (67) and (68) give rise to an $S^{1}$-moduli problem as follows. The space $\mathcal{B}^{0}$ is the quotient

$$
\mathcal{B}^{0}:=\frac{\left\{(B, \Theta) \in \mathcal{A}(E) \times \Omega^{0}(X, E) \mid(67)\right\}}{\mathcal{G}_{X 0}},
$$

the bundle $\mathcal{E}^{0} \rightarrow \mathcal{B}^{0}$ is given by

$$
\mathcal{E}^{0}:=\frac{\mathcal{B}^{0} \times \Omega^{0,1}(X, E)}{\mathcal{G}_{X 0}},
$$

and the section $\mathcal{S}^{0}: \mathcal{B}^{0} \rightarrow \mathcal{E}^{0}$ is

$$
\mathcal{S}^{0}(B, \Theta):=\bar{\partial}_{B} \Theta .
$$

Since $\Theta \neq 0$ the first equation in (67) implies the second equation. Hence the zero set of $\mathcal{S}^{0}$ is the space of gauge equivalence classes of solutions $(B, \Theta)$ of $(67)$ and (68).

At first glance $\mathcal{S}^{0}$ doesn't look like a Fredholm section. Note, however, that $\mathcal{S}^{0}$ is a two-dimensional Cauchy-Riemann operator in disguise. The condition $\bar{\partial}_{B} \Theta=0$ assserts, at the same time, that the restriction of $(B, \Theta) \in$ $\mathcal{B}^{0}$ to every slice $\{z\} \times S$ belongs to the finite dimensional manifold $M_{S}$ and that, as a function $P \rightarrow M_{S}$, this map is a solution of the (two dimensional) symplectic vortex equations. Hence, with appropriate Sobolev completions, the triple $\left(\mathcal{B}^{0}, \mathcal{E}^{0}, \mathcal{S}^{0}\right)$ is a regular $S^{1}$-moduli problem and

$$
\Phi_{k, \Sigma}^{M_{S}, \mu_{S}}\left(0, \pi_{S}^{*} c^{m} ; \mathrm{pt}\right)=\chi^{\mathcal{B}^{0}, \mathcal{E}^{0}, \mathcal{S}^{0}}\left(\pi_{0}^{*} c^{m}\right),
$$


where $\pi_{0}: \mathcal{B}^{0} \times_{S^{1}} \mathrm{E} S^{1} \rightarrow \mathrm{B} S^{1}$ is the projection.

A morphism from $\left(\mathcal{B}^{0}, \mathcal{E}^{0}, \mathcal{S}^{0}\right)$ to $\left(\mathcal{B}^{\mathrm{SW}}, \mathcal{E}^{\mathrm{SW}}, \mathcal{S}^{\mathrm{SW}}\right)$ can be defined as follows. The group of complex gauge transformations $g: X \rightarrow \mathbb{C}^{*}$ acts on the space of solutions of (67) via

$$
g^{*}(B, \Theta):=\left(B+g^{-1} \bar{\partial} g-\bar{g}^{-1} \partial \bar{g}, g^{-1} \Theta\right) .
$$

Given a pair $(B, \Theta) \in \mathcal{B}^{0}$, we look for a complex gauge transformation of the form $g=e^{f}$, where $f: X \rightarrow \mathbb{R}$, such that the triple $\left(B_{f}, \Theta_{f}, 0\right):=$ $\left(e^{f}\right)^{*}(B, \Theta, 0)$, given by

$$
B_{f}=B+\bar{\partial} f-\partial f, \quad \Theta_{f}=e^{-f} \Theta_{0},
$$

satisfies equation (63):

$$
i\left(F_{B_{f}}\right)_{\Omega}+\frac{\left|\Theta_{f}\right|^{2}}{2}=\tau .
$$

A short computation yields

$$
2 i \partial \bar{\partial} f=-d_{\Sigma}^{*} d_{\Sigma} f \mathrm{dvol}_{\Sigma}-d_{S}^{*} d_{S} f \mathrm{dvol}_{S},
$$

where $d_{S}: \Omega^{0}(S) \rightarrow \Omega^{1}(S)$ and $d_{\Sigma}: \Omega^{0}(\Sigma) \rightarrow \Omega^{1}(\Sigma)$ are the respective differentials and $d_{S}^{*}$ and $d_{\Sigma}^{*}$ their $L^{2}$-adjoints. Therefore $(2 i \partial \bar{\partial} f)_{\Omega}=-d^{*} d f$, and equation (63) for $\left(B_{f}, \Theta_{f}, 0\right)$ is equivalent to the Kazdan-Warner equation

$$
-d^{*} d f+\frac{|\Theta|^{2}}{2} e^{-2 f}=\tau-i\left(F_{B}\right)_{\Omega} .
$$

It follows from the theorem of Kazdan and Warner ([18], see also Appendix A) that this equation has a unique solution $f$ whenever

$$
\tau>\frac{1}{\operatorname{Vol}(\Sigma) \operatorname{Vol}(S)} \int_{\Sigma \times S} i F_{B_{0}} \wedge \Omega=\frac{2 \pi k}{\operatorname{Vol}(\Sigma)}+\frac{2 \pi d}{\operatorname{Vol}(S)}
$$

(see (64)). So we have constructed a map

$$
\mathcal{B}^{0} \rightarrow \mathcal{B}^{\mathrm{SW}}:(B, \Theta) \mapsto\left(B_{f}, \Theta_{f}, 0\right) .
$$

We claim that the image of this map is the submanifold of all triples of the form $(B, \Theta, 0) \in \mathcal{B}^{\mathrm{SW}}$.

A left inverse $\mathcal{B}^{\mathrm{SW}} \rightarrow \mathcal{B}^{0}$ can be constructed as follows. Given a triple $(B, \Theta, 0) \in \mathcal{B}^{\mathrm{SW}}$ we must find a complex gauge transformation of the form $g=e^{f}$, where $f: X \rightarrow \mathbb{R}$, such that the pair $\left(B_{f}, \Theta_{f}\right)$, given by

$$
B_{f}:=B+\bar{\partial} f-\partial f, \quad \Theta_{f}:=e^{-f} \Theta,
$$


satisfies (67):

$$
\frac{1}{\operatorname{Vol}(S)} \int_{S} * i F_{B_{f}}+* i\left(F_{B_{f}} \wedge \mathrm{dvol}_{\Sigma}\right)+\frac{\left|\Theta_{f}\right|^{2}}{2}=\tau .
$$

This translates into the equation

$$
-d_{\Sigma}^{*} d_{\Sigma} f_{\Sigma}-d_{S}^{*} d_{S} f+e^{-2 f} \frac{|\Theta|^{2}}{2}=\tau-\frac{1}{\operatorname{Vol}(S)} \int_{S} * i F_{B}-* i\left(F_{B} \wedge \operatorname{dvol}_{\Sigma}\right),
$$

where

$$
f_{\Sigma}:=\frac{1}{\operatorname{Vol}(S)} \int_{S} f \mathrm{dvol}_{S}: \Sigma \rightarrow \mathbb{R}
$$

By Theorem A.1 of the appendix, this equation has a unique solution $f \in$ $C^{0}\left(\Sigma, W^{2, p}(S)\right)$. If $B$ and $\Theta$ are smooth one checks easily that $f$ is smooth. This shows that for every pair $(B, \Theta, 0) \in \mathcal{B}^{\mathrm{SW}}$ there exists a unique complex gauge transformation of the form $g=e^{f}$ such that $g^{*}(B, \Theta) \in \mathcal{B}^{0}$. That this map is a left inverse of the map $\mathcal{B}^{0} \rightarrow \mathcal{B}^{\text {SW }}$ follows from the uniqueness statement in Theorem A.1: Let $(B, \Theta) \in \mathcal{B}^{0}$ and $g=e^{f}$ be a complex gauge transformation such that $g^{*}(B, \Theta) \in \mathcal{B}^{0}$. Then $f$ satisfies the equation

$$
-d_{\Sigma}^{*} d_{\Sigma} f_{\Sigma}-d_{S}^{*} d_{S} f+e^{-2 f} \frac{|\Theta|^{2}}{2}=\frac{|\Theta|^{2}}{2}
$$

and $f=0$ by uniqueness.

It follows that the map $\mathcal{B}^{0} \rightarrow \mathcal{B}^{\mathrm{SW}}$ defines an embedding of Fréchet manifolds and lifts naturally to an embedding of $\mathcal{E}^{0}$ into $\mathcal{E}^{\mathrm{SW}}$ which intertwines the two sections and idenitifies the kernels and cokernels of the linearized operators along the zero set of $\mathcal{S}^{0}$. The proof of [7, Theorem 7.4] shows that there exists a finite dimensional reduction $\left(B^{0}, E^{0}, S^{0}\right)$ of $\left(\mathcal{B}^{0}, \mathcal{E}^{0}, \mathcal{S}^{0}\right)$ in the smooth category (not involving Sobolev completions). The composition of the inclusion $B^{0} \rightarrow \mathcal{B}^{0}$ with the inclusion $\mathcal{B}^{0} \rightarrow \mathcal{B}^{\mathrm{SW}}$ (and of their lifts to the vector bundles) now defines a morphism of $S^{1}$-moduli problems as in Definition 6.2. With this established, the result follows from the (Functoriality) axiom for the Euler class.

Remark 10.6. There should be an analogue of Theorem 10.2 in the case where the product $\Sigma \times S$ is replaced by a topological Lefschetz fibration $X \rightarrow S^{2}$ on a symplectic manifold $[8,9]$. Here $\Sigma$ should be replaced by $S^{2}$ and $S$ by the generic fibre of $X$. To carry this out one has to overcome several major technical difficulties. The interesting case is where the degree $d$ of the bundle over the fibre satisfies $d \leq 2 g_{S}-2$, and so the 
space $M_{S}$ has singularities. Moroever, one has to deal with the singularities of the fibration as in [10]. In addition, the complex techniques with the Kazdan-Warner equation only work in the Kähler case. In the nonintegrable case the correspondence between the Seiberg-Witten equations (62), (63) and the symplectic vortex equations (67), (68) is much more subtle and requires a hard adiabatic limit analysis as in the proof of the Atiyah-Floer conjecture [11] or as in [15] (see [30] for an outline of the Seiberg-Witten analogue). If this program can be carried out then, combined with the work of Donaldson-Smith in [10], it might lead to an alternative proof of Taubes' theorem [33, 34, 35] about the relation between the Seiberg-Witten and the Gromov invariants.

\section{Appendix.}

\section{A. The coupled Kazdan-Warner equation.}

Let $\left(\Sigma, J_{\Sigma}, \operatorname{dvol}_{\Sigma}\right)$ and $\left(S, J_{S}, \mathrm{dvol}_{S}\right)$ be compact connected Riemann surfaces. Fix a constant $p>1$. Given a function $u \in L^{p}(\Sigma \times S)$ we define $u_{\Sigma} \in L^{p}(\Sigma)$ by

$$
u_{\Sigma}(z):=\frac{1}{\operatorname{Vol}(S)} \int_{S} u(z, \cdot) \operatorname{dvol}_{S}
$$

for $z \in \Sigma$. In the following we shall denote by $d_{S}: \Omega^{0}(S) \rightarrow \Omega^{1}(S)$ and $d_{\Sigma}: \Omega^{0}(\Sigma) \rightarrow \Omega^{1}(\Sigma)$ the respective differentials and by $d_{S}^{*}$ and $d_{\Sigma}^{*}$ their $L^{2}$-adjoint operators.

Theorem A.1. Let $p>1$ and $f, h \in C^{0}(\Sigma \times S)$ such that

$$
h \geq 0, \quad \int_{\Sigma \times S} h>0, \quad \int_{\Sigma \times S} f>0 .
$$

Then there exists a unique function $u \in C^{0}\left(\Sigma, W^{2, p}(S)\right)$ such that $u_{\Sigma} \in$ $W^{2, p}(\Sigma)$ and

$$
d_{\Sigma}^{*} d_{\Sigma} u_{\Sigma}+d_{S}^{*} d_{S} u+e^{u} h=f .
$$

Moreover, if $h$ and $f$ are smooth then so is the unique solution $u$ of (69).

The proof of the theorem is based on a lemma and two propositions. 
Lemma A.2. Let $S$ be a compact Riemann surface. Then there exists a constant $c_{S}>0$ such that the following holds. Let $p>1, C \geq 0$, and $0<a \leq A$. If $h \in C^{0}(S)$ and $u \in W^{2, p}(S)$ satisfy

$$
h \geq 0, \quad h_{0}:=\frac{1}{\operatorname{Vol}(S)} \int_{S} h \operatorname{dvol}_{S}>0,
$$

and

$$
a-e^{u} h \leq d_{S}^{*} d_{S} u \leq A-f^{-1}\left(e^{u}\right) h
$$

almost everywhere, where $f(r):=r e^{C r}$, then

$$
\log \left(\frac{a}{\|h\|_{L^{\infty}}}\right) \leq u \leq \log \left(\frac{A}{h_{0}}\right)+\frac{A}{h_{0}}\left(C+c_{S}\|h\|_{L^{\infty}}\right) .
$$

Proof. Assume first that $u$ and $h$ are smooth. Choose $c_{S}>0$ such that

$$
\int_{S} v \operatorname{dvol}_{S}=0 \quad \Longrightarrow \quad 2\|v\|_{L^{\infty}} \leq c_{S}\left\|d_{S}^{*} d_{S} v\right\|_{L^{\infty}}
$$

for every $v \in C^{\infty}(S)$. Let $v \in C^{\infty}(S)$ be the unique solution of the equation

$$
d_{S}^{*} d_{S} v=h-h_{0}, \quad \int_{S} v \mathrm{dvol}_{S}=0 .
$$

Since $\left\|h-h_{0}\right\|_{L^{\infty}} \leq\|h\|_{L^{\infty}}$ it follows that

$$
\max _{S} v-\min _{S} v \leq c_{S}\|h\|_{L^{\infty}} .
$$

Now fix a constant $\varepsilon>0$ and denote

$$
w_{\varepsilon}:=\frac{A+\varepsilon}{h_{0}} v+u \text {. }
$$

Choose $x_{\varepsilon} \in S$ such that $w_{\varepsilon}\left(x_{\varepsilon}\right)=\sup _{S} w_{\varepsilon}$. Then

$$
\begin{aligned}
0 & \leq d_{S}^{*} d_{S} w_{\varepsilon}\left(x_{\varepsilon}\right) \\
& =\frac{A+\varepsilon}{h_{0}} d_{S}^{*} d_{S} v\left(x_{\varepsilon}\right)+d_{S}^{*} d_{S} u\left(x_{\varepsilon}\right) \\
& \leq \frac{A+\varepsilon}{h_{0}}\left(h\left(x_{\varepsilon}\right)-h_{0}\right)+A-h\left(x_{\varepsilon}\right) f^{-1}\left(e^{u\left(x_{\varepsilon}\right)}\right) \\
& =-\varepsilon+h\left(x_{\varepsilon}\right)\left(\frac{A+\varepsilon}{h_{0}}-f^{-1}\left(e^{u\left(x_{\varepsilon}\right)}\right)\right) .
\end{aligned}
$$


It follows that $h\left(x_{\varepsilon}\right)>0$ and $f^{-1}\left(e^{u\left(x_{\varepsilon}\right)}\right)<(A+\varepsilon) / h_{0}$. Since $f$ is strictly monotone, this implies

$$
u\left(x_{\varepsilon}\right)<\log \left(f\left(\frac{A+\varepsilon}{h_{0}}\right)\right)=\log \left(\frac{A+\varepsilon}{h_{0}}\right)+C \frac{A+\varepsilon}{h_{0}} .
$$

Since $w_{\varepsilon}(x) \leq w_{\varepsilon}\left(x_{\varepsilon}\right)$ for all $x \in S$ it follows that

$$
\begin{aligned}
u(x) & \leq u\left(x_{\varepsilon}\right)+\frac{A+\varepsilon}{h_{0}}\left(v\left(x_{\varepsilon}\right)-v(x)\right) \\
& \leq \log \left(\frac{A+\varepsilon}{h_{0}}\right)+\frac{A+\varepsilon}{h_{0}}\left(C+\max _{S} v-\min _{S} v\right) \\
& \leq \log \left(\frac{A+\varepsilon}{h_{0}}\right)+\frac{A+\varepsilon}{h_{0}}\left(C+c_{S}\|h\|_{L^{\infty}}\right) .
\end{aligned}
$$

This holds for every $\varepsilon>0$ and every $x \in S$. Hence

$$
\sup _{S} u \leq \log \left(\frac{A}{h_{0}}\right)+\frac{A}{h_{0}}\left(C+c_{S}\|h\|_{L^{\infty}}\right) .
$$

To prove the first inequality we choose $x_{0} \in S$ such that $u\left(x_{0}\right)=\inf _{S} u$. Then

$$
0 \geq d_{S}^{*} d_{S} u\left(x_{0}\right) \geq a-e^{u\left(x_{0}\right)} h\left(x_{0}\right) \geq a-e^{u\left(x_{0}\right)}\|h\|_{L^{\infty}}
$$

and hence

$$
\inf _{S} u=u\left(x_{0}\right) \geq \log \left(\frac{a}{\|h\|_{L^{\infty}}}\right) .
$$

This proves the lemma in the smooth case.

Now suppose that $h \in C^{0}(S)$ and $u \in W^{2, p}(S)$ satisfy the hypotheses of the lemma. Then $u$ is continuous and (70) shows that $d_{S}^{*} d_{S} u \in L^{\infty}(S)$. Choose sequences $a_{\nu} \rightarrow a$ and $A_{\nu} \rightarrow A$ such that

$$
0<a_{\nu}<a \leq A<A_{\nu}
$$

Then there exist sequences of smooth functions $u_{\nu}, h_{\nu} \in C^{\infty}(S)$ such that $h_{\nu}$ converges uniformly to $h, u_{\nu}$ converges to $u$ in the $W^{2, p}$-norm, $h_{\nu} \geq 0$, and

$$
a_{\nu}-e^{u_{\nu}} h_{\nu} \leq d_{S}^{*} d_{S} u_{\nu} \leq A_{\nu}-f^{-1}\left(e^{u_{\nu}} h_{\nu}\right) .
$$

To see this, we may first choose a sequence $w_{\nu} \in C^{\infty}(S)$ converging to $d_{S}^{*} d_{S} u$ in the $L^{p}$ norm and satisfying $a_{\nu}-e^{u} h<w_{\nu}<A_{\nu}-f^{-1}\left(e^{u} h\right)$. Then define $u_{\nu}$ as the solution of the equation $d_{S}^{*} d_{S} u_{\nu}=w_{\nu}$ with $\int_{S}\left(u_{\nu}-u\right) \mathrm{dvol}_{S}=0$ and choose any sequence $h_{\nu} \in C^{\infty}(S)$ converging uniformly to $h$ to obtain 
the required estimate for $u_{\nu}$ and $h_{\nu}$. It then follows that $u_{\nu}$ and $h_{\nu}$ satisfy the hypotheses of the lemma with $a$ and $A$ replaced by $a_{\nu}$ and $A_{\nu}$, respectively. Hence they satisfy the conclusion and so the required estimate for $u$ and $h$ follows by taking the limit $\nu \rightarrow \infty$.

Proposition A.3. Let $p>1$. For every $t \in \mathbb{R}$ and every $h \in C^{0}(S)$ such that $h \geq 0$ there exists a unique solution $u \in W^{2, p}(S)$ of the equation

$$
d_{S}^{*} d_{S} u+e^{u} h=\frac{1}{\operatorname{Vol}(S)} \int_{S} e^{u} h \operatorname{dvol}_{S}, \quad \frac{1}{\operatorname{Vol}(S)} \int_{S} u \operatorname{dvol}_{S}=t .
$$

Moreover, if $h \in W^{k, p}(S)$ for some integer $k \geq 1$ then $u \in W^{k+2, p}(S)$. If $k p>2$ then the map $(h, t) \mapsto u$ which assigns to each pair $(h, t) \in$ $W^{k, p}(S) \times \mathbb{R}$ that satisfies $h \geq 0$ the unique solution $u \in W^{k+2, p}(S)$ of (71) extends to a smooth map between open subsets of Banach spaces.

Proof. The proof has three steps.

Step 1. For every $p>1$ and every $c>0$ there exists a constant $c_{p}>0$ such that, if $h \in C^{0}(S)$ and $t \in \mathbb{R}$ satisfy

$$
h \geq 0, \quad h_{0}:=\frac{1}{\operatorname{Vol}(S)} \int_{S} h \operatorname{dvol}_{S}>\frac{1}{c}, \quad\|h\|_{L^{\infty}} \leq c, \quad|t| \leq c,
$$

then

$$
\|u\|_{W^{2, p}} \leq c_{p}
$$

for every solution $u \in W^{2, p}(S)$ of (71).

Let $u$ be a solution of (71) and denote

$$
a:=\frac{1}{\operatorname{Vol}(S)} \int_{S} e^{u} h \mathrm{dvol}_{S} .
$$

Then, by (72) and Lemma A.2 with $C=0$ and $A=a$, we have

$$
\log \left(\frac{a}{c}\right) \leq u \leq \log \left(\frac{a}{h_{0}}\right)+\frac{c c_{S} a}{h_{0}} .
$$

Integrating the first inequality over $S$ gives

$$
\log \left(\frac{a}{c}\right) \leq \frac{1}{\operatorname{Vol}(S)} \int_{S} u \operatorname{dvol}_{S}=t \leq c
$$


and hence $a \leq c e^{c}$. Moreover,

$$
e^{u} \leq \frac{a}{h_{0}} e^{c c_{S} a / h_{0}} \leq a c e^{c^{2} c_{S} a} \leq c^{2} e^{c} e^{c^{3} c_{S} e^{c}} .
$$

Hence $e^{u}$ satisfies a uniform upper bound, depending only on $S$ and $c$. Hence there exists a constant $c^{\prime}=c^{\prime}(c)>0$ such that $\left\|d_{S}^{*} d_{S} u\right\|_{L^{\infty}} \leq c^{\prime}$ for every solution of (71). Since $\operatorname{Vol}(S)^{-1} \int_{S} u \mathrm{dvol}_{S}=t \in[-c, c]$, Step 1 follows from elliptic regularity for the Laplace operator on $S$.

Step 2. Consider the Banach spaces

$$
\mathcal{X}:=W^{2, p}(S), \quad \mathcal{Y}:=L_{0}^{p}(S) \times \mathbb{R},
$$

where $L_{0}^{p}(S)$ denotes the space of $L^{p}$-functions on $S$ with mean value zero. For $h \in C^{0}(S)$ define $\mathcal{F}_{h}: \mathcal{X} \rightarrow \mathcal{Y}$ by

$$
\mathcal{F}_{h}(u):=\left(d_{S}^{*} d_{S} u+e^{u} h-\frac{1}{\operatorname{Vol}(S)} \int_{S} e^{u} h \operatorname{dvol}_{S}, \frac{1}{\operatorname{Vol}(S)} \int_{S} u \operatorname{dvol}_{S}\right) .
$$

If $h \geq 0$ then the differential $\mathcal{F}_{h}(u): \mathcal{X} \rightarrow \mathcal{Y}$ is a Banach space isomorphism for every $u \in \mathcal{X}$.

The differential of $\mathcal{F}_{h}$ is given by

$$
d \mathcal{F}_{h}(u) \xi=\left(d_{S}^{*} d_{S} \xi+e^{u} h \xi-\frac{1}{\operatorname{Vol}(S)} \int_{S} e^{u} h \xi \operatorname{dvol}_{S}, \frac{1}{\operatorname{Vol}(S)} \int_{S} \xi \operatorname{dvol}_{S}\right) .
$$

Hence $d \mathcal{F}_{h}(u): \mathcal{X} \rightarrow \mathcal{Y}$ is a Fredholm operator of index zero. Multiplying the first component of $d \mathcal{F}_{h}(u) \xi$ by $\xi$ and integrating over $S$ we find that the kernel of $d \mathcal{F}_{h}(u)$ consists of all functions $\xi \in W^{2, p}(S)$ that satisfy

$$
\int_{S}\left|d_{S} \xi\right|^{2} \operatorname{dvol}_{S}+\int_{S} e^{u} h|\xi|^{2} \operatorname{dvol}_{S}=0, \quad \int_{S} \xi \operatorname{dvol}_{S}=0 .
$$

Hence $d \mathcal{F}_{h}(u)$ is bijective whenever $h \geq 0$.

Step 3. We prove the proposition.

If $h=0$ then every solution of (71) is constant and hence $u \equiv t$ is the only solution. Now assume $h=1$ and let $u \in W^{2, p}(S)$ be a solution of (71). Then

$$
d_{S}^{*} d_{S} u+e^{u}=\frac{1}{\operatorname{Vol}(S)} \int_{S} e^{u} \operatorname{dvol}_{S}
$$

and hence, by Lemma A.2,

$$
e^{u} \geq \frac{1}{\operatorname{Vol}(S)} \int_{S} e^{u} \mathrm{dvol}_{S}
$$


This implies $e^{u} \equiv$ constant and hence $u \equiv t$. Thus we have proved the existence and uniqueness statament in the cases $h=0$ and $h=1$. Now let $h \in C^{0}(S)$ be any nonnegative function such that $\int_{S} h \mathrm{dvol}_{S}>0$, and define

$$
h_{\varepsilon}:=(1-\varepsilon) h+\varepsilon .
$$

We prove that the number of solutions of (71) with $h$ replaced by $h_{\varepsilon}$ is independent of $\varepsilon$. To see this consider the set

$$
\mathcal{M}:=\left\{(\varepsilon, u) \mid 0 \leq \varepsilon \leq 1, u \in W^{2, p}(S), \mathcal{F}_{h_{\varepsilon}}(u)=(0, t)\right\} .
$$

By Step 2, this set is a smooth 1-manifold with boundary and the projection $\mathcal{M} \rightarrow[0,1]:(\varepsilon, u) \mapsto \varepsilon$ is a submersion. That $\mathcal{M}$ is compact follows from the fact that, by Step 1, there exists a constant $c_{p}>0$ such that

$$
(\varepsilon, u) \in \mathcal{M} \quad \Longrightarrow \quad\|u\|_{W^{2, p}} \leq c_{p}
$$

Hence every sequence $\left(\varepsilon_{i}, u_{i}\right) \in \mathcal{M}$ has a subsequence such that $u_{i}$ converges in $C^{0}(S)$ and $\varepsilon_{i}$ converges. Hence, for this subsequence, $e^{u_{i}} h_{\varepsilon_{i}}$ converges in $C^{0}(S)$ and so, by elliptic regularity for the Laplace operator on $S, u_{i}$ converges in $W^{2, p}(S)$ (for any $p>1$ ). Thus $\mathcal{M}$ is compact and so the number $\# \mathcal{F}_{h_{\varepsilon}}^{-1}(0, t)$ is independent of $\varepsilon \in[0,1]$. For $\varepsilon=1$ this number is one and this proves the existence and uniqueness statement. That $h \in W^{k, p}$ implies $u \in W^{k+2, p}$ follows from elliptic regularity for the Laplace operator. That the map $(h, t) \mapsto u$ is smooth follows from the implicit function theorem and Step 2.

Proposition A.4. Let $a>0$ and $\mathcal{H} \subset C^{0}(\Sigma \times S)$ be a compact set such that $h \geq 0$ for every $h \in \mathcal{H}$. Then there exists a constant $\delta=\delta(\Sigma, S, \mathcal{H}, a)>0$ such that the following holds. If $p>1$ and $u \in C^{0}\left(\Sigma, W^{2, p}(S)\right)$ satisfies $u_{\Sigma} \in W^{2, p}(\Sigma)$ and

$$
d_{\Sigma}^{*} d_{\Sigma} u_{\Sigma}+d_{S}^{*} d_{S} u+e^{u} h=a
$$

for some $h \in \mathcal{H}$ then $d_{\Sigma}^{*} d_{\Sigma} u_{\Sigma}$ is continuous and

$$
\delta h_{\Sigma} \leq a-d_{\Sigma}^{*} d_{\Sigma} u_{\Sigma} \leq \delta^{-1} \sup _{S} h, \quad \delta \leq e^{u} \leq \delta^{-1} .
$$

Proof. The proof has three steps.

Step 1. Let $c_{S}$ be the constant of Lemma A.2 and choose $c>0$ such that

$$
h \in \mathcal{H} \quad \Longrightarrow \quad\|h\|_{L^{\infty}} \leq c .
$$


Define $f:[0, \infty) \rightarrow[0, \infty)$ by $f(r):=r e^{c c_{S} r}$. Then

$$
f^{-1}\left(e^{u}\right) h_{\Sigma} \leq a-d_{\Sigma}^{*} d_{\Sigma} u_{\Sigma} \leq e^{u} \sup _{S} h
$$

for every $h \in \mathcal{H}$ and every solution $u$ of $(73)$.

Integrating (73) over $S$ we obtain

$$
a-d_{\Sigma}^{*} d_{\Sigma} u_{\Sigma}=\frac{1}{\operatorname{Vol}(S)} \int_{S} e^{u} h \mathrm{dvol}_{S} \geq 0
$$

In particular, $d_{\Sigma}^{*} d_{\Sigma} u_{\Sigma}$ is continuous. If $\left.h\right|_{\{z\} \times S} \equiv 0$ then $a=d_{\Sigma}^{*} d_{\Sigma} u_{\Sigma}(z)$ and $h_{\Sigma}(z)=0$. So the assertion of Step 1 holds trivially at the point $z$. If $\left.h\right|_{\{z\} \times S} \not \equiv 0$ then $a-d_{\Sigma}^{*} d_{\Sigma} u_{\Sigma}(z)>0$ and so the restrictions of $h$ and $u$ to $\{z\} \times S$ satisfy the requirements of Lemma A.2 with $C=0$ and $a=A$ replaced by the constant $a-d_{\Sigma}^{*} d_{\Sigma} u_{\Sigma}(z)$. Hence

$$
\log \left(\frac{a-d_{\Sigma}^{*} d_{\Sigma} u_{\Sigma}}{\sup _{S} h}\right) \leq u \leq \log \left(\frac{a-d_{\Sigma}^{*} d_{\Sigma} u_{\Sigma}}{h_{\Sigma}}\right)+\frac{c c_{S}\left(a-d_{\Sigma}^{*} d_{\Sigma} u_{\Sigma}\right)}{h_{\Sigma}}
$$

This implies the assertion of Step 1 in the case $h_{\Sigma}(z) \neq 0$.

Step 2. There exists a constant $\delta=\delta(\Sigma, S, \mathcal{H}, a)>0$ such that

$$
\delta \leq e^{u_{\Sigma}} \leq \delta^{-1}
$$

for every $h \in \mathcal{H}$ and every solution $u$ of $(73)$.

By the proof of Step 1, we have

$$
\log \left(\frac{a-d_{\Sigma}^{*} d_{\Sigma} u_{\Sigma}}{c}\right) \leq u_{\Sigma} \leq \log \left(\frac{a-d_{\Sigma}^{*} d_{\Sigma} u_{\Sigma}}{h_{\Sigma}}\right)+\frac{c c_{S}\left(a-d_{\Sigma}^{*} d_{\Sigma} u_{\Sigma}\right)}{h_{\Sigma}}
$$

whenever $h_{\Sigma}(z)>0$ and hence

$$
a-c e^{u_{\Sigma}} \leq d_{\Sigma}^{*} d_{\Sigma} u_{\Sigma} \leq a-f^{-1}\left(e^{u_{\Sigma}}\right) h_{\Sigma} .
$$

Hence $u_{\Sigma} \in W^{2, p}(\Sigma)$ satisfies the second inequality in (70) with $A=a$, $C=c c_{S}$, and $h$ replaced by $h_{\Sigma}$. It satisfies the first inequality with $h$ replaced by $c$. Hence, by Lemma A.2,

$$
\log \left(\frac{a}{c}\right) \leq u_{\Sigma} \leq \log \left(\frac{a \operatorname{Vol}(\Sigma)}{\int_{\Sigma} h_{\Sigma} \operatorname{dvol}_{\Sigma}}\right)+\frac{c\left(c_{S}+c_{\Sigma}\right) a \operatorname{Vol}(\Sigma)}{\int_{\Sigma} h_{\Sigma} \operatorname{dvol}_{\Sigma}} .
$$

This proves Step 2. 
Step 3. We prove the proposition.

For $h \in \mathcal{H}$ and $z \in \Sigma$ denote by $h_{z}: S \rightarrow \mathbb{R}$ the function $h_{z}(x):=h(z, x)$ and let $\mathcal{T}_{h_{z}}: \mathbb{R} \rightarrow W^{2, p}(S)$ be the map which assigns to every $t \in \mathbb{R}$ the unique solution $u=\mathcal{T}_{h_{z}}(t) \in W^{2, p}(S)$ of (71) with $h$ replaced by $h_{z}$. Then every solution $u: \Sigma \times S \rightarrow \mathbb{R}$ of (73) satisfies

$$
u(z, \cdot)=\mathcal{T}_{h_{z}}\left(u_{\Sigma}(z)\right)
$$

By Proposition A.3, the map $\mathcal{H} \times \Sigma \times \mathbb{R} \rightarrow W^{2, p}(S):(h, z, t) \mapsto \mathcal{T}_{h_{z}}(t)$ is continuous. Since $\mathcal{H}$ is compact it follows that there exists an $\varepsilon>0$ such that

$$
h \in \mathcal{H}, \quad z \in \Sigma, \quad \delta \leq e^{t} \leq \delta^{-1} \quad \Longrightarrow \quad\left\|\mathcal{T}_{h_{z}}(t)\right\|_{L^{\infty}(S)} \leq|\log (\varepsilon)| .
$$

This implies $\varepsilon \leq e^{u} \leq \varepsilon^{-1}$ for every $h \in \mathcal{H}$ and every solution $u$ of (73). The inequality for $a-d_{\Sigma}^{*} d_{\Sigma} u_{\Sigma}$ now follows from Step 1.

Proof of Theorem A.1. The proof has four steps.

Step 1. It suffices to prove the theorem if $f$ is constant.

Let $v_{\Sigma} \in W^{2, p}(\Sigma)$ be a solution of the equation

$$
d_{\Sigma}^{*} d_{\Sigma} v_{\Sigma}=f_{\Sigma}-a, \quad a:=\frac{1}{\operatorname{Vol}(\Sigma)} \int_{\Sigma} f_{\Sigma} \mathrm{dvol}_{\Sigma}
$$

and let $v \in C^{0}\left(\Sigma, W^{2, p}(S)\right)$ be the unique solution of the equation

$$
d_{S}^{*} d_{S} v=f-f_{\Sigma}, \quad \frac{1}{\operatorname{Vol}(S)} \int_{S} v \operatorname{dvol}_{S}=v_{\Sigma}
$$

This equation is understood pointwise for $z \in \Sigma$. Then $v$ is continuous and

$$
d_{\Sigma}^{*} d_{\Sigma} v_{\Sigma}+d_{S}^{*} d_{S} v=f-a .
$$

Note that if $f$ is smooth then so is $v$. Moreover, $u$ is a solution of (73) with $h$ replaced by $e^{v} h$ if and only if $u+v$ is a solution of (69).

Step 2. Let $h \in C^{0}(S)$ such that $h \geq 0$ and define $f_{h}: \mathbb{R} \rightarrow \mathbb{R}$ by

$$
f_{h}(t):=\frac{1}{\operatorname{Vol}(S)} \int_{S} e^{u} h \mathrm{dvol}_{S},
$$


where $u \in W^{2, p}(S)$ is the unique solution of $(71)$. Then $f_{h}^{\prime}(t) \geq 0$ for every $t \in \mathbb{R}$ with equality if and only if $h \equiv 0$.

Let $u \in W^{2, p}(S)$ be the unique solution of (71) and $\xi \in W^{2, p}(S)$ be the unique solution of the equation

$$
d_{S}^{*} d_{S} \xi+e^{u} h \xi=\frac{1}{\operatorname{Vol}(S)} \int_{S} e^{u} h \xi \operatorname{dvol}_{S}, \quad \frac{1}{\operatorname{Vol}(S)} \int_{S} \xi \operatorname{dvol}_{S}=1 .
$$

Then

$$
f_{h}^{\prime}(t)=\frac{1}{\operatorname{Vol}(S)} \int_{S} e^{u} h \xi \operatorname{dvol}_{S}=\frac{1}{\operatorname{Vol}(S)} \int_{S}\left(\left|d_{S} \xi\right|^{2}+e^{u} h|\xi|^{2}\right) \operatorname{dvol}_{S} \geq 0 .
$$

Equality implies that $\xi \equiv 1$ and $h \equiv 0$.

Step 3. Given a nonzero continuous function $h: \Sigma \times S \rightarrow[0, \infty)$ define $\mathcal{F}_{h}: W^{2, p}(\Sigma) \rightarrow L^{p}(\Sigma)$ by

$$
\mathcal{F}_{h}\left(u_{\Sigma}\right)(z):=d_{\Sigma}^{*} d_{\Sigma} u_{\Sigma}(z)+f_{h_{z}}\left(u_{\Sigma}(z)\right)
$$

for $z \in \Sigma$, where $h_{z}:=h(z, \cdot) \in C^{0}(S)$. Then $d \mathcal{F}_{h}\left(u_{\Sigma}\right): W^{2, p}(\Sigma) \rightarrow L^{p}(\Sigma)$ is a Banach space isomorphism for every $u_{\Sigma} \in W^{2, p}(\Sigma)$.

This follows directly from Step 2.

Step 4. We prove the theorem.

By Step 1 we may assume $f \equiv a$. Assume first that $h \equiv 1$. We claim that in this case $u \equiv \log (a)$ is the only solution of (73). To see this, note that, by Proposition A.3, the restriction of $u$ to each fibre $\{z\} \times S$ is constant, hence $u=u_{\Sigma}$ and $d_{\Sigma}^{*} d_{\Sigma} u_{\Sigma}+e^{u}=a$, and hence, again by Proposition A.3, $u=$ $u_{\Sigma}=\log (a)$. Now let $h \in C^{0}(\Sigma \times S)$ be any nonzero nonnegative function. Note that $\mathcal{F}_{h}\left(u_{\Sigma}\right)=a$ iff $u$ is a solution of (73). Define $h_{\varepsilon} \in C^{0}(\Sigma \times S)$ by $h_{\varepsilon}:=(1-\varepsilon) h+\varepsilon$ and consider the set $\mathcal{M} \subset[0,1] \times W^{2, p}(\Sigma)$ given by

$$
\mathcal{M}:=\left\{\left(\varepsilon, u_{\Sigma}\right) \mid 0 \leq \varepsilon \leq 1, u_{\Sigma} \in W^{2, p}(\Sigma), \mathcal{F}_{h_{\varepsilon}}\left(u_{\Sigma}\right)=a\right\} .
$$

By Step 3, this is a 1-manifold with boundary and the projection $\mathcal{M} \rightarrow$ $[0,1]:\left(\varepsilon, u_{\Sigma}\right) \mapsto \varepsilon$ is a submersion. To prove that $\mathcal{M}$ is compact note that, by Proposition A.4, there exists a constant $c>0$ such that

$$
\left\|u_{\Sigma}\right\|_{W^{2, p}} \leq c
$$

for every $\left(\varepsilon, u_{\Sigma}\right) \in \mathcal{M}$. Hence every sequence $\left(\varepsilon_{i}, u_{i}\right) \in \mathcal{M}$ has a subsequence such that $\varepsilon_{i}$ converges and $u_{i}$ converges in $C^{0}(\Sigma)$. The equation

$$
d_{\Sigma}^{*} d_{\Sigma} u_{i}(z)+f_{h_{\varepsilon_{i}, z}}\left(u_{i}(z)\right)=a
$$


now shows that $u_{i}$ converges in $W^{2, p}(\Sigma)$. Hence $\mathcal{M}$ is compact and so the number $\# \mathcal{F}_{h_{\varepsilon}}^{-1}(a)$ is independent of $\varepsilon$. For $\varepsilon=1$ we have seen that this number is one. This proves the existence and uniqueness statement.

Now suppose that $h$ is smooth. Then the function $\Sigma \times \mathbb{R} \rightarrow \mathbb{R}:(z, t) \mapsto$ $f_{h_{z}}(t)$ is smooth and hence, by a standard elliptic bootstrapping argument, the unique solution $u_{\Sigma}: \Sigma \rightarrow \mathbb{R}$ of the equation

$$
d_{\Sigma}^{*} d_{\Sigma} u_{\Sigma}(z)+f_{h_{z}}\left(u_{\Sigma}(z)\right)=a
$$

is smooth. Hence, by Lemma A.2, the unique solution $u: \Sigma \times S \rightarrow \mathbb{R}$ of the equation

$$
d_{S}^{*} d_{S} u+e^{u} h=\frac{1}{\operatorname{Vol}(S)} \int_{S} e^{u} h \operatorname{dvol}_{S}, \quad \frac{1}{\operatorname{Vol}(S)} \int_{S} u \operatorname{dvol}_{S}=u_{\Sigma},
$$

is smooth. This proves the theorem.

\section{B. The local slice theorem.}

Let $G$ be a compact Lie group and $P \rightarrow X$ be a principal $G$-bundle over a compact $n$-manifold $X$. For $p>n / 2$ denote by $\mathcal{G}^{k+1, p}=\mathcal{G}^{k+1, p}(P)$ the space of all $W^{k+1, p_{-}}$sections of the bundle $P \times_{\text {ad }} \mathrm{G} \rightarrow X$. Fix a smooth reference connection $\hat{A} \in \mathcal{A}(P)$ and denote by

$$
\mathcal{A}^{1, p}(P):=\left\{\hat{A}+\alpha \mid \alpha \in W^{1, p}\left(X, T^{*} X \otimes \mathfrak{g}_{P}\right)\right\}
$$

the space of $W^{1, p}$-connections. This space is independent of the connection $\hat{A}$.

Theorem B.1. Let $p, q$ be positive real number such that

$$
q \geq p>\frac{n}{2}, \quad q>n, \quad \text { if } p \leq n \text { then } q<\frac{n p}{n-p} .
$$

Then, for every $A_{0} \in \mathcal{A}^{1, p}(P)$ and every positive constant $c_{0}$, there exist positive constants $c$ and $\delta$ such that the following holds. If $A \in \mathcal{A}^{1, p}(P)$ satisfies

$$
\left\|A-A_{0}\right\|_{W^{1, p}} \leq c_{0}, \quad\left\|A-A_{0}\right\|_{L^{q}} \leq \delta
$$

then there exists a gauge transformation $g \in \mathcal{G}^{2, p}(P)$ such that

$$
d_{A_{0}}^{*}\left(g^{*} A-A_{0}\right)=0
$$

and

$$
\left\|g^{*} A-A\right\|_{L^{q}} \leq c\left\|A-A_{0}\right\|_{L^{q}}, \quad\left\|g^{*} A-A\right\|_{W^{1, p}} \leq c\left\|A-A_{0}\right\|_{W^{1, p}} .
$$


Lemma B.2. Let $p, q, r$ be positive real numbers such that

$$
r \leq p, \quad r \leq q, \quad \frac{1}{p}+\frac{1}{q}<\frac{1}{n}+\frac{1}{r} .
$$

Then there exists a constant $c>0$ such that

$$
\|f g\|_{W^{1, r}} \leq c\|f\|_{W^{1, p}}\|g\|_{W^{1, q}} .
$$

for $f, g \in C_{0}^{\infty}\left(\mathbb{R}^{n}\right)$. In particular, this holds when $p$ and $q$ satisfy (74) and $r=p$. It also holds when $p=q=r>n$.

Proof. By Hölder's inequality and the product rule, we have

$$
\|f g\|_{W^{1, r}} \leq\|f\|_{W^{1, p}}\|g\|_{L^{r p /(p-r)}}+\|f\|_{L^{r q /(q-r)}}\|g\|_{W^{1, q}} .
$$

If $q>n$ the Sobolev embedding theorem asserts that the $L^{r p /(p-r)}$-norm of $g$ can be estimated from above by the $W^{1, q}$-norm. The same holds for $q=n$ since then it follows from the hypotheses that $p>r$. If $q<n$ we have $r<p$ and

$$
\frac{r p}{p-r}=\left(\frac{1}{r}-\frac{1}{p}\right)^{-1}<\left(\frac{1}{q}-\frac{1}{n}\right)^{-1}=\frac{n q}{n-q}
$$

and hence the $L^{r p /(p-r)}$-norm of $g$ can again be estimated from above by the $W^{1, q}$-norm. Similarly, the $L^{r q /(q-r)}$-norm of $f$ can again be estimated from above by the $W^{1, p}$-norm.

Lemma B.3. If $A \in \mathcal{A}^{1, p}(P)$ and $p>n / 2$ then the following holds for every $r>1$.

(i) If $p<n$ assume in addition $r<n p /(n-p)$. Then the operator $d_{A}$ : $\mathrm{W}^{1, r}\left(X, \mathfrak{g}_{P}\right) \rightarrow L^{r}\left(X, T^{*} X \otimes \mathfrak{g}_{P}\right)$ is a compact perturbation of $d_{\hat{A}}$. Similarly for $d_{A}^{*}$.

(ii) For $r \leq p$ the operator $d_{A}: \mathrm{W}^{2, r}\left(X, \mathfrak{g}_{P}\right) \rightarrow W^{1, r}\left(X, T^{*} X \otimes \mathfrak{g}_{P}\right)$ is a compact perturbation of $d_{\hat{A}}$. Similarly for $d_{A}^{*}$.

(iii) For $r \leq p$ the operator $d_{A}^{*} d_{A}: \mathrm{W}^{2, r}\left(X, \mathfrak{g}_{P}\right) \rightarrow L^{r}\left(X, \mathfrak{g}_{P}\right)$ is a compact perturbation of $d_{\hat{A}}^{*} d_{\hat{A}}$.

Proof. For $\xi \in \Omega^{0}\left(X, \mathfrak{g}_{P}\right)$ and $\alpha \in \Omega^{1}\left(X, \mathfrak{g}_{P}\right)$ we have

$$
d_{A} \xi-d_{\hat{A}} \xi=[(A-\hat{A}), \xi], \quad d_{A}^{*} \alpha-d_{\hat{A}}^{*} \alpha=*[*(A-\hat{A}) \wedge \alpha] .
$$


Assume first that $p<n$. Then $r<n p /(n-p)$ and hence there exists a real number $s>1$ such that $1 / s+(n-p) / n p=1 / r$. Since $2 p<n$ it follows that $s<n r /(n-r)$ whenever $r<n$. Hence the Sobolev embedding theorem asserts that the inclusion $W^{1, r}\left(X, \mathfrak{g}_{P}\right) \hookrightarrow L^{s}\left(X, \mathfrak{g}_{P}\right)$ is compact. It also asserts that $A-\hat{A} \in L^{n p /(n-p)}\left(X, T^{*} X \otimes \mathfrak{g}_{P}\right)$ and hence, by Hölder's inequality, the operator $L^{s}\left(X, \mathfrak{g}_{P}\right) \rightarrow L^{r}\left(X, T^{*} X \otimes \mathfrak{g}_{P}\right): \xi \mapsto[(A-\hat{A}), \xi]$ is bounded. Hence the composition with the inclusion $W^{1, r} \hookrightarrow L^{s}$ is compact. If $p \geq n$ choose any number $s>r$ such that the inclusion $W^{1, r}\left(X, \mathfrak{g}_{P}\right) \hookrightarrow$ $L^{s}\left(X, \mathfrak{g}_{P}\right)$ is compact and use the fact that $A-\hat{A} \in L^{r s /(s-r)}\left(X, T^{*} X \otimes \mathfrak{g}_{P}\right)$. This proves (i).

We prove (ii). By Lemma B.2 the operator

$$
W^{1, s}\left(X, \mathfrak{g}_{P}\right) \rightarrow W^{1, r}\left(X, T^{*} X \otimes \mathfrak{g}_{P}\right): \xi \mapsto[(A-\hat{A}), \xi]
$$

is bounded whenever $r \leq p, r \leq s$, and $1 / p+1 / s<1 / n+1 / r$. If $r>n$ then $p>n$ and we may choose $s=r$. If $r \leq n$ then, since $2 p>n$, we have

$$
\frac{1}{p}+\frac{n-r}{n r}<\frac{1}{n}+\frac{1}{r}
$$

and hence may choose $s$ such that $r \leq s<n r /(n-r)$. In either case the Sobolev embedding theorem asserts that the inclusion $W^{2, r}\left(X, \mathfrak{g}_{P}\right) \hookrightarrow$ $W^{1, s}\left(X, \mathfrak{g}_{P}\right)$ is compact. This proves (ii). Assertion (iii) follows directly from (i) and (ii).

Lemma B.4. Suppose $p$ and $q$ satisfy (74) and let $A \in \mathcal{A}^{1, p}(P)$. Then there exists a constant $c=c(A)=c(A, p, q)>0$ such that, for every $\alpha \in$ $W^{1, p}\left(X, T^{*} X \otimes \mathfrak{g}_{P}\right)$, there exists a $\xi \in W^{2, p}\left(X, \mathfrak{g}_{P}\right)$ such that

$$
d_{A}^{*} d_{A} \xi=d_{A}^{*} \alpha
$$

and

$$
\|\xi\|_{W^{2, p}} \leq c\left\|d_{A}^{*} \alpha\right\|_{L^{p}}, \quad\|\xi\|_{W^{1, q}} \leq c\|\alpha\|_{L^{q}}
$$

Proof. Let $r:=q /(q-1)$ so that

$$
\frac{1}{q}+\frac{1}{r}=1
$$

By Lemma B.3, the operator $d_{A}: W^{1, s}\left(X, \mathfrak{g}_{P}\right) \rightarrow L^{s}\left(X, T^{*} X \otimes \mathfrak{g}_{P}\right)$ is a compact perturbation of $d_{\hat{A}}$ for $s=q$ and $s=r$. Let

$$
W^{-1, q}\left(X, \mathfrak{g}_{P}\right):=\left(W^{1, r}\left(X, \mathfrak{g}_{P}\right)\right)^{*}
$$


and denote by $d_{A}^{*}: L^{q}\left(X, T^{*} X \otimes \mathfrak{g}_{P}\right) \rightarrow W^{-1, q}\left(X, \mathfrak{g}_{P}\right)$ the dual operator of $d_{A}: W^{1, r}\left(X, \mathfrak{g}_{P}\right) \rightarrow L^{r}\left(X, T^{*} X \otimes \mathfrak{g}_{P}\right)$. Then

$$
d_{A}^{*} d_{A}: W^{1, q}\left(X, \mathfrak{g}_{P}\right) \rightarrow W^{-1, q}\left(X, \mathfrak{g}_{P}\right)
$$

is a compact perturbation of $d_{\hat{A}}^{*} d_{\hat{A}}$ and hence is a Fredholm operator of index zero. Likewise, it follows from Lemma B.3 that the operator

$$
d_{A}^{*} d_{A}: W^{2, p}\left(X, \mathfrak{g}_{P}\right) \rightarrow L^{p}\left(X, \mathfrak{g}_{P}\right)
$$

is a compact perturbation of $d_{\hat{A}}^{*} d_{\hat{A}}$ and hence is also a Fredholm operator of index zero. The operator (77) is a natural extension of (78). Taking the $L^{2}$-inner product of $d_{A}^{*} d_{A} \xi$ with $\xi$ for $\xi \in W^{2, p}\left(X, \mathfrak{g}_{P}\right)$ we see that the kernel of (78) is the finite dimensional subspace

$$
H^{0}(X, A):=\operatorname{ker} d_{A} \subset W^{2, p}\left(X, \mathfrak{g}_{P}\right)
$$

The operator (77) has the same kernel, because every $\xi \in W^{1, q}\left(X, \mathfrak{g}_{P}\right)$ with $d_{A} \xi=0$ lies in $W^{2, p}\left(X, \mathfrak{g}_{P}\right)$. Choose a complement $E^{\prime}$ of $H^{0}(X, A)$ in the Sobolev space $W^{1, q}\left(X, \mathfrak{g}_{P}\right)$. Then

$$
E:=E^{\prime} \cap W^{2, p}\left(X, \mathfrak{g}_{P}\right)
$$

is a complement of $H^{0}(X, A)$ in $W^{2, p}\left(X, \mathfrak{g}_{P}\right)$. Let $F^{\prime}$ denote the image of the operator $d_{A}^{*}: L^{q}\left(X, T^{*} X \otimes \mathfrak{g}_{P}\right) \rightarrow W^{-1, q}\left(X, \mathfrak{g}_{P}\right)$ and $F$ denote the image of the operator $d_{A}^{*}: W^{1, p}\left(X, T^{*} X \otimes \mathfrak{g}_{P}\right) \rightarrow L^{p}\left(X, \mathfrak{g}_{P}\right)$. Then $H^{0}(X, A) \subset$ $W^{1, r}\left(X, \mathfrak{g}_{P}\right)$ annihilates $F^{\prime}$ and is $L^{2}$-orthogonal to $F$. Moreover $F^{\prime}$ contains the image of (77) and $F$ contains the image of (78). Hence, for dimensional reasons, $F^{\prime}$ is equal to the image of (77) and $F$ is equal to the image of (78). Thus $d_{A}^{*} d_{A}$ is a Banach space isomorphism from $E$ to $F$ and extends to a Banach space isomorphism from $E^{\prime}$ to $F^{\prime}$

Now let $\alpha \in W^{1, p}\left(X, T^{*} X \otimes \mathfrak{g}_{P}\right)$. Then $d_{A}^{*} \alpha \in F$ and hence there exists a unique $\xi \in E$ that satisfies (75). By the open mapping theorem, this solution of (75) satisfies

$$
\|\xi\|_{W^{2, p}} \leq\left\|\left(d_{A}^{*} d_{A}\right)^{-1}\right\|_{\mathcal{L}(F, E)}\left\|d_{A}^{*} \alpha\right\|_{L^{p}}
$$

Since $\xi \in E^{\prime}$ it also satisfies

$$
\|\xi\|_{W^{1, q}} \leq\left\|\left(d_{A}^{*} d_{A}\right)^{-1}\right\|_{\mathcal{L}\left(F^{\prime}, E^{\prime}\right)}\left\|d_{A}^{*} \alpha\right\|_{W^{-1, q}}
$$


Now

$$
\begin{aligned}
\left\|d_{A}^{*} \alpha\right\|_{W^{-1, q}} & =\sup _{\eta \neq 0} \frac{\left\langle\eta, d_{A}^{*} \alpha\right\rangle_{W^{1, r}, W^{-1, q}}}{\|\eta\|_{W^{1, r}}} \\
& =\sup _{\eta \neq 0} \frac{\left\langle d_{A} \eta, \alpha\right\rangle_{L^{r}, L^{q}}}{\|\eta\|_{W^{1, r}}} \\
& \leq \sup _{\eta \neq 0} \frac{\left\|d_{A} \eta\right\|_{L^{r}}\|\alpha\|_{L^{q}}}{\|\eta\|_{W^{1, r}}} \\
& \leq c\|\alpha\|_{L^{q}},
\end{aligned}
$$

where the constant $c$ depends only on $A$ and $r$. Hence $\xi$ satisfies (76).

Lemma B.5. Suppose $p$ and $q$ satisfy (74) and fix a constant $c_{0}>0$. Then there exists a constant $c=c\left(c_{0}, p, q\right)>0$ such that the following holds. If $\xi \in W^{2, p}\left(X, \mathfrak{g}_{P}\right)$ satisfies $\|\xi\|_{W^{2, p}} \leq c_{0}$ then, for every $A \in \mathcal{A}^{1, p}(P)$, we have

$$
\begin{gathered}
\left\|\exp (\xi)^{*} A-A-d_{A} \xi\right\|_{W^{1, p}} \leq c\left(1+\|A-\hat{A}\|_{W^{1, p}}\right)\|\xi\|_{W^{1, q}}\|\xi\|_{W^{2, p}}, \\
\left\|\exp (\xi)^{*} A-A\right\|_{W^{1, p}} \leq c\left(1+\|A-\hat{A}\|_{W^{1, p}}\right)\|\xi\|_{W^{2, p}} \\
\left\|\exp (\xi)^{*} A-A\right\|_{L^{q}} \leq c\left(1+\|A-\hat{A}\|_{W^{1, p}}\right)\|\xi\|_{W^{1, q}} .
\end{gathered}
$$

Proof. The function $\alpha(t):=\exp (t \xi)^{*} A-A$ satisfies the differential equation $\dot{\alpha}(t)=d_{A} \xi-[\xi, \alpha(t)]$ and $\alpha(0)=0$. Hence

$$
\alpha(t)=\sum_{k=0}^{\infty} \frac{(-1)^{k} t^{k+1}}{(k+1) !} \operatorname{ad}(\xi)^{k} d_{A} \xi,
$$

and hence

$$
\exp (\xi)^{*} A-A-d_{A} \xi=\sum_{k=1}^{\infty} \frac{(-1)^{k}}{(k+1) !} \operatorname{ad}(\xi)^{k} d_{A} \xi
$$

Now

$$
\begin{aligned}
\left\|d_{A} \xi\right\|_{L^{q}} & \leq\left\|d_{\hat{A}} \xi\right\|_{L^{q}}+\|[(A-\hat{A}), \xi]\|_{L^{q}} \\
& \leq\left\|d_{\hat{A}} \xi\right\|_{L^{q}}+\|A-\hat{A}\|_{L^{q}}\|\xi\|_{L^{\infty}} \\
& \leq c\left(1+\|A-\hat{A}\|_{W^{1, p}}\right)\|\xi\|_{W^{1, q}}
\end{aligned}
$$


and, by Lemma B.2,

$$
\begin{aligned}
\left\|d_{A} \xi\right\|_{W^{1, p}} & \leq\left\|d_{\hat{A}} \xi\right\|_{W^{1, p}}+\|[(A-\hat{A}), \xi]\|_{W^{1, p}} \\
& \leq\left\|d_{\hat{A}} \xi\right\|_{W^{1, p}}+c^{\prime}\|A-\hat{A}\|_{W^{1, p}}\|\xi\|_{W^{1, q}} \\
& \leq c^{\prime \prime}\left(1+\|A-\hat{A}\|_{W^{1, p}}\right)\|\xi\|_{W^{2, p}} .
\end{aligned}
$$

Hence the assertion follows from (79) and Lemma B.2.

Proof of Theorem B.1. The proof is by Newton's iteration.

Step 1. Fix a connection $A_{0} \in \mathcal{A}^{1, p}(P)$ and a constant $c_{0}>0$. Let $c\left(A_{0}\right)$ be the constant of Lemma B.4 with $A$ replaced by $A_{0}$. Then there exists a constant $c_{1}=c_{1}\left(A_{0}, c_{0}\right)$ such that the following holds. If $A \in \mathcal{A}^{1, p}(P)$ such that

$$
\left\|A-A_{0}\right\|_{W^{1, p}} \leq c_{0}
$$

and $\xi \in W^{2, p}\left(X, \mathfrak{g}_{P}\right)$ is a solution of the equation

$$
d_{A_{0}}^{*} d_{A_{0}} \xi=d_{A_{0}}^{*}\left(A_{0}-A\right)
$$

such that

(80)

$$
\|\xi\|_{W^{2, p}} \leq c\left(A_{0}\right)\left\|d_{A_{0}}^{*}\left(A-A_{0}\right)\right\|_{L^{p}}, \quad\|\xi\|_{W^{1, q}} \leq c\left(A_{0}\right)\left\|A-A_{0}\right\|_{L^{q}},
$$

then $g:=\exp (\xi)$ and $A_{1}:=g^{*} A$ satisfy

$$
\begin{gathered}
\left\|A_{1}-A_{0}\right\|_{L^{q}}+\left\|d_{A_{0}}^{*}\left(A_{1}-A_{0}\right)\right\|_{L^{p}} \leq c_{1}\left\|A-A_{0}\right\|_{L^{q}}, \\
\left\|A_{1}-A_{0}\right\|_{W^{1, p}} \leq c_{1}\left\|A-A_{0}\right\|_{W^{1, p}} .
\end{gathered}
$$

By Lemma B.3 and (80), we have

$$
\|\xi\|_{W^{2, p}} \leq c_{2}\left\|A-A_{0}\right\|_{W^{1, p}} \leq c_{0} c_{2} .
$$

for some constant $c_{2}=c_{2}\left(A_{0}\right)>0$. Now let $c_{3}$ be the constant of Lemma B.5, with $A$ replaced by $A_{0}$ and $c_{0}$ replaced by $c_{0} c_{2}$. Then

$$
\begin{aligned}
\left\|A_{1}-A\right\|_{W^{1, p}} & =\left\|g^{*} A-A\right\|_{W^{1, p}} \\
& \leq c_{3}\left(1+\|A-\hat{A}\|_{W^{1, p}}\right)\|\xi\|_{W^{2, p}} \\
& \leq c_{2} c_{3}\left(1+c_{0}+\left\|A_{0}-\hat{A}\right\|_{W^{1, p}}\right)\left\|A-A_{0}\right\|_{W^{1, p}} \\
& \leq c_{4}\left\|A-A_{0}\right\|_{W^{1, p}}
\end{aligned}
$$


for some constant $c_{4}=c_{4}\left(A_{0}, c_{0}\right)$. This proves (82). Similarly,

$$
\left\|A_{1}-A\right\|_{L^{q}} \leq c_{5}\left\|A-A_{0}\right\|_{L^{q}} .
$$

for some constant $c_{5}=c_{5}\left(A_{0}, c_{0}\right)$.

Now consider the identity

$$
\begin{aligned}
d_{A_{0}}^{*}\left(A_{1}-A_{0}\right) & =d_{A_{0}}^{*}\left(g^{*} A-A_{0}\right) \\
& =d_{A_{0}}^{*}\left(g^{*} A-A\right)+d_{A_{0}}^{*}\left(A-A_{0}\right) \\
& =d_{A_{0}}^{*}\left(g^{*} A-A-d_{A_{0}} \xi\right) \\
& =d_{A_{0}}^{*}\left(g^{*} A-A-d_{A} \xi\right)+d_{A_{0}}^{*}\left[\left(A-A_{0}\right) \wedge \xi\right] .
\end{aligned}
$$

We have

$$
d_{A_{0}}^{*}\left[\left(A-A_{0}\right) \wedge \xi\right]=\left[d_{A_{0}}^{*}\left(A-A_{0}\right) \wedge \xi\right]+*\left[d_{A_{0}} \xi \wedge *\left(A-A_{0}\right)\right] .
$$

Let $r:=q p /(q-p)$ so that

$$
\frac{1}{q}+\frac{1}{r}=\frac{1}{p}
$$

If $p<n$ then $r<n p /(n-p)$ and hence there is a Sobolev embedding $W^{2, p} \hookrightarrow W^{1, r}$. For $p \geq n$ such an embedding exists as well. Hence, in either case,

$$
\begin{aligned}
& \left\|d_{A_{0}}^{*}\left[\left(A-A_{0}\right) \wedge \xi\right]\right\|_{L^{p}} \\
& \leq c_{6}\left(\left\|d_{A_{0}}^{*}\left(A-A_{0}\right)\right\|_{L^{p}}\|\xi\|_{L^{\infty}}+\left\|A-A_{0}\right\|_{L^{q}}\left\|d_{A_{0}} \xi\right\|_{L^{r}}\right) \\
& \leq c_{7}\left(\left\|d_{A_{0}}^{*}\left(A-A_{0}\right)\right\|_{L^{p}}\|\xi\|_{W^{1, q}}+\left\|A-A_{0}\right\|_{L^{q}}\|\xi\|_{W^{2, p}}\right) \\
& \leq c_{8}\left\|A-A_{0}\right\|_{W^{1, p}}\left\|A-A_{0}\right\|_{L^{q}} .
\end{aligned}
$$

Moreover, it follows from Lemma B.5 that

$$
\begin{aligned}
\left\|d_{A_{0}}^{*}\left(g^{*} A-A-d_{A} \xi\right)\right\|_{L^{p}} & \leq c_{9}\left\|g^{*} A-A-d_{A} \xi\right\|_{W^{1, p}} \\
& \leq c_{10}\left(1+\|A-\hat{A}\|_{W^{1, p}}\right)\|\xi\|_{W^{2, p}}\|\xi\|_{W^{1, q}} \\
& \leq c_{11}\left\|A-A_{0}\right\|_{W^{1, p}}\left\|A-A_{0}\right\|_{L^{q}} .
\end{aligned}
$$

These two estimates imply

$$
\left\|d_{A_{0}}^{*}\left(A_{1}-A_{0}\right)\right\|_{L^{p}} \leq c_{0}\left(c_{8}+c_{11}\right)\left\|A-A_{0}\right\|_{L^{q}}
$$


and this proves (81).

Step 2. Define the sequence $A_{2}, A_{3}, \ldots$ inductively by

$$
A_{\nu+1}=g_{\nu}^{*} A_{\nu}, \quad g_{\nu}=\exp \left(\xi_{\nu}\right),
$$

where $\xi_{\nu} \in W^{2, p}\left(X, \mathfrak{g}_{P}\right)$ is chosen such that

$$
d_{A_{0}}{ }^{*} d_{A_{0}} \xi_{\nu}=d_{A_{0}}{ }^{*}\left(A_{0}-A_{\nu}\right)
$$

and, with $c=c\left(A_{0}\right)$,

$$
\left\|\xi_{\nu}\right\|_{W^{2, p}} \leq c\left\|d_{A_{0}}^{*}\left(A_{\nu}-A_{0}\right)\right\|_{L^{p}}, \quad\left\|\xi_{\nu}\right\|_{W^{1, q}} \leq c\left\|A_{\nu}-A_{0}\right\|_{L^{q}}
$$

There exist constants $\delta>0$ and $c_{12}>0$ such that the following holds. If

$$
\left\|A-A_{0}\right\|_{L^{q}}<\delta
$$

then, for every $\nu \geq 1$,

$$
\begin{aligned}
\left\|d_{A_{0}}^{*}\left(A_{\nu}-A_{0}\right)\right\|_{L^{p}} & \leq 2^{1-\nu} c_{1}\left\|A-A_{0}\right\|_{L^{q}} \\
\left\|A_{\nu}-A_{0}\right\|_{W^{1, p}} & \leq 2 c_{0} c_{1} \\
\left\|d_{A_{0}}^{*}\left(A_{\nu+1}-A_{0}\right)\right\|_{L^{p}} & \leq c_{12}\left\|A_{\nu}-A_{0}\right\|_{L^{q}}\left\|d_{A_{0}}{ }^{*}\left(A_{\nu}-A_{0}\right)\right\|_{L^{p}} .
\end{aligned}
$$

For $\nu=1$ the inequalities (84) and (85) were established in Step 1. Let $\nu \geq 1$ and assume, by induction, that (84) and (85) have been established with $\nu$ replaced by $j \in\{1, \ldots, \nu\}$. We prove first that (86) holds under these assumptions. As in the proof of Step 1, we have

$$
d_{A_{0}}^{*}\left(A_{\nu+1}-A_{0}\right)=d_{A_{0}}^{*}\left(g_{\nu}^{*} A_{\nu}-A_{\nu}-d_{A_{\nu}} \xi_{\nu}\right)+d_{A_{0}}^{*}\left[\left(A_{\nu}-A_{0}\right) \wedge \xi_{\nu}\right]
$$

and

$$
\begin{aligned}
& \left\|d_{A_{0}}^{*}\left[\left(A_{\nu}-A_{0}\right) \wedge \xi_{\nu}\right]\right\|_{L^{p}} \\
& \leq c_{13}\left(\left\|d_{A_{0}}^{*}\left(A_{\nu}-A_{0}\right)\right\|_{L^{p}}\left\|\xi_{\nu}\right\|_{W^{1, q}}+\left\|A_{\nu}-A_{0}\right\|_{L^{q}}\left\|\xi_{\nu}\right\|_{W^{2, p}}\right) \\
& \leq c_{14}\left\|A_{\nu}-A_{0}\right\|_{L^{q}}\left\|d_{A_{0}}^{*}\left(A_{\nu}-A_{0}\right)\right\|_{L^{p}} .
\end{aligned}
$$

It follows from (83), the induction hypothesis, and Lemma B.3, that

$$
\begin{aligned}
\left\|\xi_{j}\right\|_{W^{2, p}} & \leq c\left(A_{0}\right)\left\|d_{A_{0}}^{*}\left(A_{j}-A_{0}\right)\right\|_{L^{p}} \\
& \leq c^{\prime}\left(A_{0}\right)\left\|A_{j}-A_{0}\right\|_{W^{1, p}} \\
& \leq 2 c_{0} c_{1} c^{\prime}\left(A_{0}\right)
\end{aligned}
$$


for $j=1, \ldots, \nu$. Now we can apply Lemma B.5, with $c_{0}$ replaced by $2 c_{0} c_{1} c^{\prime}\left(A_{0}\right)$, to obtain, for some positive constant $c_{15}=c_{15}\left(A_{0}, c_{0}\right)$,

$$
\begin{aligned}
& \left\|d_{A_{0}}^{*}\left(g_{\nu}{ }^{*} A_{\nu}-A_{\nu}-d_{A_{\nu}} \xi_{\nu}\right)\right\|_{L^{p}} \\
& \leq c_{15}\left(1+\left\|A_{\nu}-\hat{A}\right\|_{W^{1, p}}\right)\|\xi\|_{W^{1, q}}\|\xi\|_{W^{2, p}} \\
& \leq c_{16}\left\|A_{\nu}-A_{0}\right\|_{L^{q}}\left\|d_{A_{0}}^{*}\left(A-A_{0}\right)\right\|_{L^{p}}
\end{aligned}
$$

where $c_{16}:=c\left(A_{0}\right)^{2} c_{15}\left(1+2 c_{0} c_{1}+\left\|A_{0}-\hat{A}\right\|_{W^{1, p}}\right)$. Hence (86) holds with $c_{12}:=c_{14}+c_{16}$.

Now we prove that (85) holds with $\nu$ replaced by $\nu+1$. By (87), the section $\xi=\xi_{j}$ satisfies the hypotheses of Lemma B.5 for $j=1, \ldots, \nu$, with $c_{0}$ replaced by $2 c_{0} c_{1} c^{\prime}\left(A_{0}\right)$. Hence

$$
\begin{aligned}
\left\|A_{j+1}-A_{j}\right\|_{W^{1, p}} & \leq c_{15}\left(1+\left\|A_{j}-\hat{A}\right\|_{W^{1, p}}\right)\left\|\xi_{j}\right\|_{W^{2, p}} \\
& \leq c\left(A_{0}\right) c_{15}\left(1+\left\|A_{j}-\hat{A}\right\|_{W^{1, p}}\right)\left\|d_{A_{0}}^{*}\left(A_{j}-A_{0}\right)\right\|_{L^{p}}
\end{aligned}
$$

for $j=1, \ldots, \nu$. Hence, by (84),

$$
\left\|A_{j+1}-A_{j}\right\|_{W^{1, p}} \leq c_{17} 2^{-j}\left\|A-A_{0}\right\|_{L^{q}} .
$$

for $j=1, \ldots, \nu$, where $c_{17}:=2 c\left(A_{0}\right) c_{15}\left(1+2 c_{0} c_{1}+\left\|A_{0}-\hat{A}\right\|_{W^{1, p}}\right) c_{1}$. If

$$
c_{17}\left\|A-A_{0}\right\|_{L^{q}} \leq c_{0} c_{1}
$$

then

$$
\begin{aligned}
\left\|A_{\nu+1}-A_{0}\right\|_{W^{1, p}} & \leq \sum_{j=1}^{\nu}\left\|A_{j+1}-A_{j}\right\|_{W^{1, p}}+\left\|A_{1}-A_{0}\right\|_{W^{1, p}} \\
& \leq c_{17}\left\|A-A_{0}\right\|_{L^{q}}+c_{1}\left\|A-A_{0}\right\|_{W^{1, p}} \\
& \leq 2 c_{0} c_{1} .
\end{aligned}
$$

This proves (85) with $\nu$ replaced by $\nu+1$.

Now we shall use (86) and the induction hypothesis to prove that (84) holds with $\nu$ replaced by $\nu+1$. Since (88) holds for $j=1, \ldots, \nu-1$, we 
have

$$
\begin{aligned}
\left\|A_{\nu}-A_{0}\right\|_{L^{q}} & \leq \sum_{j=1}^{\nu-1}\left\|A_{j+1}-A_{j}\right\|_{L^{q}}+\left\|A_{1}-A_{0}\right\|_{L^{q}} \\
& \leq c_{18} \sum_{j=1}^{\nu-1}\left\|A_{j+1}-A_{j}\right\|_{W^{1, p}}+\left\|A_{1}-A_{0}\right\|_{L^{q}} \\
& \leq\left(c_{17} c_{18}+c_{1}\right)\left\|A-A_{0}\right\|_{L^{q}} .
\end{aligned}
$$

Here $c_{18}$ is the constant in the Sobolev embedding $W^{1, p} \hookrightarrow L^{q}$. If

$$
c_{12}\left(c_{17} c_{18}+c_{1}\right)\left\|A-A_{0}\right\|_{L^{q}} \leq \frac{1}{2}
$$

then, by (86),

$$
\begin{aligned}
\left\|d_{A_{0}}^{*}\left(A_{\nu+1}-A_{0}\right)\right\|_{L^{p}} & \leq c_{12}\left\|A_{\nu}-A_{0}\right\|_{L^{q}}\left\|d_{A_{0}}^{*}\left(A_{\nu}-A_{0}\right)\right\|_{L^{p}} \\
& \leq c_{12}\left(c_{17} c_{18}+c_{1}\right)\left\|A-A_{0}\right\|_{L^{q}}\left\|d_{A_{0}}^{*}\left(A_{\nu}-A_{0}\right)\right\|_{L^{p}} \\
& \leq \frac{1}{2}\left\|d_{A_{0}}^{*}\left(A_{\nu}-A_{0}\right)\right\|_{L^{p}} .
\end{aligned}
$$

This proves (84) with $\nu$ replaced by $\nu+1$.

Step 3. We prove the theorem.

By (88), the sequence $A_{\nu}$ converges strongly in the $W^{1, p}$-norm and the limit connection

$$
A_{\infty}:=\lim _{\nu \rightarrow \infty} A_{\nu} \in \mathcal{A}^{1, p}(P)
$$

satisfies

$$
\left\|A_{\infty}-A_{0}\right\|_{W^{1, p}} \leq \sum_{j=0}^{\infty}\left\|A_{j+1}-A_{j}\right\|_{W^{1, p}} \leq\left(c_{1}+c_{17} c_{18}\right)\left\|A-A_{0}\right\|_{W^{1, p}} .
$$

Moreover, by (89),

$$
\left\|A_{\infty}-A_{0}\right\|_{L^{q}} \leq\left(c_{1}+c_{17} c_{18}\right)\left\|A-A_{0}\right\|_{L^{q}}
$$

and, by (84),

$$
d_{A_{0}}^{*}\left(A_{\infty}-A_{0}\right)=\lim _{\nu \rightarrow \infty} d_{A_{0}}^{*}\left(A_{\nu}-A_{0}\right)=0 .
$$

Write

$$
A_{\nu}=h_{\nu}^{*} A, \quad h_{\nu}=g g_{1} g_{2} \cdots g_{\nu}
$$


Consider the identity

$$
d h_{\nu}=h_{\nu} A_{\nu}-A h_{\nu}
$$

in a local frame. The right hand side of (90) is bounded in $L^{q}$ and hence $h_{\nu}$ is bounded in $W^{1, q}$. Now the product inequality of Lemma B.2 with $r=p$ shows that $h_{\nu} A_{\nu}-A h_{\nu}$ is bounded in $W^{1, p}$ and, by (90), $h_{\nu}$ is bounded in $W^{2, p}$. Hence $h_{\nu}$ has a subsequence, still denoted by $h_{\nu}$, which converges in the $W^{1, q}$-norm. Since $A_{\nu}$ converges in the $W^{1, p}$-norm it follows from Lemma B.2 with $r=p$ that $h_{\nu} A_{\nu}-A h_{\nu}$ converges in the $W^{1, p}$-norm. By $(90), h_{\nu}$ converges in the $W^{2, p}$-norm. The limit

$$
h_{\infty}:=\lim _{\nu \rightarrow \infty} h_{\nu} \in \mathcal{G}^{2, p}(P)
$$

satisfies

$$
A_{\infty}=\lim _{\nu \rightarrow \infty} h_{\nu}^{*} A=h_{\infty}^{*} A .
$$

This proves the theorem.

\section{References.}

[1] M. Audin, The Topology of Torus Actions on Symplectic Manifolds, Birkhäuser, Basel, 1991.

[2] A. Bertram, G. Daskalopoulos and R. Wentworth, Gromov invariants for holomorphic maps from Riemann surfaces into Grassmannians, J. Amer. Math. Soc., 9 (1996), 529-571.

[3] S. Bradlow, Vortices in holomorphic line bundles over closed Kähler manifolds, Comm. Math. Phys., 135 (1990), 1-17.

[4] S. Bradlow, Special metrics and stability for holomorphic bundles with global sections, J. Diff. Geom., 33 (1991), 169-213.

[5] S. Bradlow, G. Daskalopoulos and R. Wentworth, Birational equivalence of vortex moduli, Topology, 35 (1996), 731-748.

[6] K. Cieliebak, A.R. Gaio and D.A. Salamon, J-holomorphic curves, moment maps, and invariants of Hamiltonian group actions, IMRN, 10 (2000), 831-882. 
[7] K. Cieliebak, I. Mundet i Riera and D.A. Salamon, Equivariant moduli problems and the Euler class, preprint, ETH-Zürich, October 2001, to appear in Topology.

[8] S.K. Donaldson, Symplectic submanifolds and almost complex geometry, J. Diff. Geom., 44 (1996), 666-705.

[9] S.K. Donaldson, Lefschetz fibrations on symplectic manifolds, preprint, 1999, to appear in J. Diff. Geom..

[10] S.K. Donaldson and I. Smith, Lefschetz pencils and the canonical class for symplectic 4-manifolds, preprint, December 2000, math. SG/0012067.

[11] S. Dostoglou and D.A. Salamon, Self-dual instantons and holomorphic curves, Annals of Math., 139 (1994), 581-640.

[12] Y. Eliashberg and M. Gromov, Convex symplectic manifolds, in 'Several Complex Variables and Complex Geometry' (Santa Cruz, CA, 1989), Part 2, Proc. Sympos. Pure Math., 52, Amer. Math. Soc., Providence, RI, 1991, 135-162.

[13] K. Fukaya and K. Ono, Arnold conjecture and Gromov-Witten invariants for general symplectic manifolds, preprint, February 1996, Summary in 'Proceddings of the Taniguchi Conference on Mathematics', edited by T. Sunada and M. Maruyama, Advanced Studies in Pure Math., 31, Math. Society of Japan, 2001, 75-91.

[14] A.R. Gaio, J-holomorphic curves and moment maps, Ph.D. thesis, University of Warwick, 1999.

[15] A.R. Gaio and D.A. Salamon, J-holomorphic curves, moment maps, and adiabatic limits, preprint, June 2001.

[16] O. Garcia-Prada, A direct existence proof for the vortex equations over a compact Riemann surface, Bulletin L.M.S., 26 (1994), 88-96.

[17] M. Gromov, Pseudo holomorphic curves in symplectic manifolds, Invent. Math., 82 (1985), 307-347.

[18] J. Kazdan and F. Warner, Curvature functions for compact 2-manifolds, Ann. Math., 99 (1978), 14-47.

[19] F. Kirwan, Cohomology of Quotients in Symplectic and Algebraic Geometry, Princeton University Press, 1984. 
[20] A.M. Li, J. Robbin and Y. Ruan, Virtual Muduli Cycles and GromovWitten Invariants, in preparation.

[21] T.J. Li and A. Liu, General wall-crossing formula, Math. Res. Letters, 2 (1995), 797-810.

[22] J. Li and G. Tian, Virtual moduli cycles and Gromov-Witten invariants of algebraic varieties, J. Amer. Math. Soc., 11 (1998), 119-174.

[23] D. McDuff and D.A. Salamon, J-holomorphic Curves and Quantum Cohomology, University Lecture Series, 6, Amer. Math. Soc., Providence, RI, 1994.

[24] D. McDuff and D.A. Salamon, Introduction to Symplectic Topology, Oxford University Press, 1995.

[25] I. Mundet i Riera, Yang-Mills-Higgs theory for symplectic fibrations, Ph.D. thesis, Madrid, April 1999.

[26] I. Mundet i Riera, Hamiltonian Gromov-Witten invariants, preprint, math. SG/0002121, to appear in Topology.

[27] C. Okonek and A. Teleman, Gauge theoretical equivariant GromovWitten invariants and the full Seiberg-Witten invariants of ruled surfaces, preprint, math. SG/0102119.

[28] K. Ono and H. Ohta, Notes on symplectic 4-manifolds with $b^{+}=1$, I, II, Internat. J. of Math., 7 (1996), 755-770.

[29] Y. Ruan, Virtual neighborhoods and pseudoholomorphic curves, in 'Topics in Symplectic 4-Manifolds' (Irvine CA 1996), Internat. Press, Cambridge, MA, 1998, 101-116.

[30] D.A. Salamon, Seiberg-Witten invariants of mapping tori, symplectic fixed points, and Lefschetz numbers, Turkish Journal of Mathematics, 23 (1999), 117-143.

[31] D.A. Salamon, Spin Geometry and Seiberg-Witten Invariants, in preparation.

[32] K. Uhlenbeck, Connections with $L^{p}$ bounds on the curvature, Commun. Math. Phys., 83 (1982), 31-42.

[33] C.H. Taubes, The Seiberg-Witten and the Gromov invariants, Math. Res. Letters, 2 (1995), 221-238. 
[34] C.H. Taubes, SW $\Longrightarrow$ Gr, from the Seiberg-Witten equations to pseudoholomorphic curves, J. Amer. Math. Soc., 9 (1996), 845-918.

[35] C.H. Taubes, Seiberg-Witten and Gromov Invariants for Symplectic Four-Manifolds, International Press, 2000.

[36] M. Thaddeus, Stable pairs, linear systems, and the Verlinde algebra, Inv. Math., 117 (1994), 317-353.

[37] K. Wehrheim, Uhlenbeck Compactness: An Exposition, in preparation.

STANFORD UNIVERSITY

Fac. Ciências - Univ. Porto

Universidad Autónoma de MadRid

ETH-ZÜRICH 
